\title{
What Security Can We Achieve Within 4 Rounds?*
}

\author{
Carmit Hazay \\ Faculty of Engineering, Bar-Ilan University, Ramat Gan, Israel \\ carmit.hazay@biu.ac.il \\ Muthuramakrishnan Venkitasubramaniam \\ University of Rochester, Rochester, NY14611, USA \\ muthuv@cs.rochester.edu \\ Communicated by Hugo Krawczyk.
}

Received 24 August 2016 / Revised 14 April 2019

Online publication 22 May 2019

\begin{abstract}
Katz and Ostrovsky (Crypto 2004) proved that five rounds are necessary for stand-alone general black-box constructions of secure two-party protocols and at least four rounds are necessary if only one party needs to receive the output. Recently, Ostrovsky, Richelson and Scafuro (Crypto 2015) proved optimality of this result by showing how to realize stand-alone, secure two-party computation under general assumptions (with black-box proof of security) in four rounds where only one party receives the output, and an extension to five rounds where both parties receive the output. In this paper, we study the question of what security is achievable for stand-alone two-party protocols within four rounds and show the following results:
\end{abstract}

1. A 4-round two-party protocol for coin-tossing that achieves $1 / p$-security (i.e., simulation fails with probability at most $1 / p+$ negl), in the presence of malicious corruptions.

2. A 4-round two-party protocol for general functionalities where both parties receive the output, that achieves $1 / p$-security and privacy in the presence of malicious adversaries corrupting one of the parties, and full security in the presence of non-aborting malicious adversaries corrupting the other party.

3. A 3-round oblivious-transfer protocol that achieves $1 / p$-security against arbitrary malicious senders, while simultaneously guaranteeing a meaningful notion of privacy against malicious corruptions of either party.

4. Finally, we show that the simulation-based security guarantees for our 3-round protocols are optimal by proving that $1 / p$-simulation security is impossible to achieve against both parties in three rounds or less when requiring some minimal guarantees on the privacy of their inputs.

Keywords. Secure computation, Coin-tossing, Oblivious-transfer, Round complexity.

${ }^{*}$ Carmit Hazay: Research partially supported by a grant from the Israel Ministry of Science and Technology (Grant No. 3-10883), by the European Research Council under the ERC consolidators Grant agreement n. 615172 (HIPS), and by the BIU Center for Research in Applied Cryptography and Cyber Security in conjunction with the Israel National Cyber Bureau in the Prime Minister's Office.

Muthuramakrishnan Venkitasubramaniam: Research supported by Google Faculty Research Grant and NSF Award CNS-1526377.

(C) International Association for Cryptologic Research 2019 


\section{Introduction}

Secure two-party computation enables two parties to mutually run a protocol that computes some function $f$ on their private inputs, while preserving a number of security properties. Two of the most important properties are privacy and correctness. The former implies data confidentiality, namely nothing leaks by the protocol execution but the computed output. The latter requirement implies that the protocol enforces the integrity of the computations made by the parties, namely honest parties learn the correct output. Feasibility results are well established $[5,34,52,63]$, proving that any efficient functionality can be securely computed under full simulation-based definitions (following the ideal/real paradigm). Security is typically proven with respect to two adversarial models: the semi-honest model (where the adversary follows the instructions of the protocol but tries to learn more than it should from the protocol transcript) and the malicious model (where the adversary follows an arbitrary polynomial-time strategy), and feasibility holds in the presence of both types of attacks.

The Round Complexity of Multi-party Computation An important complexity measure of secure computation that has been extensively studied in the literature, is the round complexity of secure protocols, where by a round of communication we mean a single message transmission from one party to another. In the stand-alone setting, Yao [63] presented the first constant-round secure two-party computation protocol in the semi-honest model. In contrast, Goldreich, Micali and Wigderson [34] showed how to obtain protocols that tolerate malicious adversaries which requires non-constant number of rounds, followed by Lindell [47] who gave the first constant-round secure two-party protocol tolerating such attacks. In an important characterization, Katz and Ostrovsky [45] determined that the exact round complexity of achieving a (black-box) maliciously secure two-party computation is five (and four if only one of the parties receives an output). More precisely, they constructed a 5-round protocol to securely compute arbitrary functionalities and showed that there cannot exist any 4-round black-box construction that securely realizes the coin-tossing functionality with black-box simulation. A more recent work by Garg et al. [32] studied the round complexity of secure protocols in the simultaneous message model, where in a single round multiple parties are allowed to simultaneously transmit messages. Garg et al. extended the Katz-Ostrovsky lower bound to show that four rounds are necessary to realize the coin-tossing functionality in the multi-party setting (where all parties receive the output). While these results only consider the stand-alone model, assuming some trusted setup such as a common reference string (CRS), it is possible to construct round-optimal (i.e., two-round) secure two-party protocols; see [43] for example.

In this work, we are interested in understanding secure two-party computation with four rounds such that both parties learn their outputs and study various security relaxations and their combinations.

The Round Complexity of Oblivious-Transfer (OT) At the heart of all multi-party protocols in the dishonest majority setting lies an oblivious-transfer protocol that serves as a fundamental building block. Therefore, understanding the round complexity of multi-party protocols requires understanding the round complexity of OT protocols. In 
the Random Oracle model, Naor and Pinkas [54] developed a 2-round OT protocol that obtains one-sided simulation (w.r.t. the sender), whereas only privacy is guaranteed against a malicious receiver. Halevi and Kalai in [40] showed how to construct 2-round protocols for OT without the random-oracle where only privacy is guaranteed against both the sender and receiver. When assuming setup, the work of Peikert, Vaikuntanathan and Waters [59] shows how to construct highly efficient 2-round protocols for the OT functionality in the CRS model. In the plain model, Ostrovsky, Richelson and Scafuro [55] strengthened the [45] construction by demonstrating a 5-round protocol where additionally the underlying cryptographic primitives are used only in a "black-box" way.

When restricting to black-box simulation (and non-simultaneous messages), fully malicious secure computation cannot be achieved in less than four rounds. The main question we address in this work is:

What security is achievable for stand-alone malicious two-party computation within four rounds when both parties receive the output?

Relaxed Notions of Security In this work, we focus on what security is achievable in the standard communication model (i.e., not simultaneous message passing) in the twoparty setting. More precisely, we initiate the study of what security guarantees can be achieved in round-efficient protocols. Toward that, we begin with an overview of some relaxations to the standard notions of simulation-based security considered in this work.

1. $1 / p$-Security In our first relaxation, we weaken the indistinguishability requirement on the simulation. In the standard definition, the views of an adversary in the real and ideal world needs to be indistinguishable except with negligible probability. More concretely, in our relaxed variant, we require the two distributions to be distinguishable with probability at most $1 / p+$ negl, where $p(\cdot)$ is some arbitrary but specified polynomial. This relaxation has been considered in the past in the context of achieving coin-tossing $[17,50]$ and fairness for arbitrary functionalities [29]. We remark that there are two subtle variants of this basic notion. The worst case guarantee with such a security notion can allow an adversary to completely violate the privacy of honest parties with $1 / p$. We present an OT protocol with $1 / p$-security where there are explicit attacks with probability $1 / p$ and one where there are no attacks. A second consideration is whether the protocol depends on $p$. Our main OT protocols depend on the polynomial $p$, while our coin-tossing protocol is independent of $p$. Chung, Liu and Pass [19] have considered a similar notion for zero-knowledge, referred to as $\epsilon$-weak zero-knowledge. Furthermore, they showed that a protocol that achieves $\epsilon$-weak zero-knowledge for an inverse polynomial $\epsilon$ implies super-polynomial simulation. We believe that a similar result can be obtained for our coin-tossing protocol, i.e., super-polynomial simulation under standard (polynomial-time hardness) assumptions. Finally, we believe that achieving $1 / p$-security can be seen as a first step for achieving super-polynomial simulation from standard assumptions [18].

2. Privacy Only Loosely speaking, privacy is a weaker notion of simulation-based definition for which no party should be able to distinguish two views generated based on distinct set of inputs for the other party but yield the same output. Private OT was formalized by Halevi and Kalai in [40] that considered two separate 
definitions. Namely, receiver privacy requires that no malicious sender be able to distinguish the cases when the receiver's input is 0 or 1 . On the other hand, sender privacy requires that for every malicious receiver and honest sender with input $\left(s_{0}, s_{1}\right)$ there exists some input $b$ for which the receiver cannot distinguish an execution where $s_{1-b}$ is set to the correct value from an execution where $s_{1-b}$ is sampled uniformly at random.

The notion of private OT is related to the notion of input-indistinguishable computation, introduced by Micali et al. in [51], which considers a weaker security notion for two-party computation. Formally, they require an "implicit input" function that can, from a transcript of the interaction, specify the input of a particular party. We prove in Appendix B that one of our protocols, in fact, can be shown to satisfy input-indistinguishable security. For simplicity, we will follow our definition for the protocols in the main body. We remark that the notion of input-indistinguishability holds for any functionality, whereas our definition is specified only for oblivious-transfer.

3. Non-aborting (malicious) Adversaries Non-aborting adversaries imply adversaries who are guaranteed to not abort in the middle of the execution. Security against non-aborting strategies implies that if an adversary deviates from the protocol it will be detected (either because of an ill-formed ${ }^{1}$ message or the adversary aborts before delivering the message). This notion is therefore stronger than semi-honest security where malicious behavior can go undetected. It is further useful in settings that apply external measures to ensure fairness, such as the recent work of [10] that has shown how to rely on external mechanisms, such as bitcoins to ensure fairness. Another line of works considers "optimistic" fairness where a trusted party can be used to compensate the loss of information due to aborting adversaries [3,49]. In this setting, the trusted party is involved only if one of the parties prematurely aborts and is not involved in the computation otherwise. In such settings, it is a reasonable assumption to develop and analyze security in the presence of non-aborting adversaries. Another motivation for considering non-aborting adversaries arises from a long line of works $[4,6,22,42,53]$ that have a preprocessing (or an offline) phase followed by an online phase. The offline phase is independent of the parties' secret inputs and generates correlated randomness to be consumed in the online phase. The key insight here is that since no private inputs are used in the first phase of the computation an aborting adversary can at best stop from the computation being performed and not violate privacy of the real inputs. Finally, we highlight that security against non-aborting adversaries where the aborting party can be identified is useful in protocols for parameter generation (e.g., common reference string). This is sufficient and useful in cryptocurrency and blockchain applications where parties want to generate ECDSA signing keys [21,48] or RSA modulus [25,41] in a distributed way.

In general, $1 / p$-security and privacy are incomparable. While privacy always guarantees some form of input-indistinguishable security, $1 / p$-secure protocols could lose complete security with probability $1 / p$. All our protocols that achieve $1 / p$-security additionally satisfies privacy. We further remark that achieving $1 / p$-security has the added benefit of easily composing with protocols to achieve a similar level security. We show how to combine our $1 / p$-secure OT protocols with the 2-round secure computation protocol

\footnotetext{
${ }^{1} \mathrm{~A}$ message is considered ill-formed if the recipient of the message rejects the message.
} 
of Ishai et al. from [43] to get secure computation of general functionalities with $1 / p$ security. Furthermore, $1 / p$-security can be easily specified for general functionalities while extending the analogous privacy notion is harder.

We also study the combination of $1 / p$-security for OT in the presence of corrupted senders and non-aborting receivers. In the case of security of non-aborting adversaries, only one of our two OT protocols violates the sender's privacy when the adversary aborts. As mentioned above, this is still meaningful in some scenarios. On the other hand, this protocol is conceptually simpler than our protocol for which an aborting receiver cannot violate the sender's privacy. Moreover, since cheating is always detected, our protocol can be used for computing random OTs where the sender's inputs are random and detecting cheating does not violate privacy. Random OTs are very useful in the context of OT extensions and triples generation [46].

Related Work Aumann and Lindell introduced the notion of covert security in [2] as a relaxation of standard simulation-based security. This notion models adversaries that may deviate arbitrarily from the protocol specification in an attempt to cheat, but do not wish to get caught doing so. They consider several formulations, one of which allows the simulator to fail, as long as it is guaranteed that the real and ideal output distributions are distinguishable with a probability that is related to the probability of detecting cheating. While the $1 / p$-security notion does not imply covert security, combining $1 / p$-security with privacy may archive a stronger notion than covert security. Roughly speaking, this is because whenever an adversary misbehaves privacy is still preserved. In contrast, covert adversary may violate privacy with some probability $p$ (but is guaranteed to get caught with a related probability, which is not ensured by $1 / p$-security). Moreover, if we restrict the class of adversaries to be non-aborting then our protocols satisfy covert security. We leave it as future work to concretely compare our definitions and protocols with covert security.

Another related notion is that of super-polynomial-time simulation $[13,56,58]$ which allows the simulator to run in super-polynomial (potentially exponential) time. In the context of zero-knowledge proofs, exponential time simulation is equivalent to witness indistinguishability. However, more generally, for secure computation it seems that the implication is only one-way where exponential time simulation implies privacy. Concretely, the protocols in $[23,40]$ and some of our protocols guarantee statistical privacy against at least one party and hence cannot admit exponential time simulation.

In the context of (partial) fairness, Gordon and Katz [29] showed how to construct secure protocols with $1 / p$-security which are fully private. The focus of their work is to achieve a meaningful notion of fairness, while the round complexity incurred by their protocols is high. We remark that our definition of privacy is weaker than the definition of [29]. While their definition has a simulation-based flavor, ours is an indistinguishabilitybased definition (where the combination of both guarantees is discussed above). Nevertheless, the focus of our work is not related to fairness rather to minimize the number of rounds. More recently, Garay et al. [30] considered a utility-based security definition that is both $1 / p$-secure and fully private (and in that sense, stronger than $1 / p$-security).

The work of Ishai et al. [43] shows how to construct a 2-round secure two-party computation protocol in the so-called OT-hybrid model, where the parties are assumed access to an ideal functionality implementing oblivious-transfer (OT). In essence, their 
work shows that improving the round complexity of secure computation is closely related to constructing round-efficient oblivious-transfer protocols.

\subsection{Our Results}

Coin-Tossing Our first result concerns with the coin-tossing functionality where we show how to achieve $1 / p$-security. More precisely, we prove the following theorem:

Theorem 1.1. (Informal) Assuming the discrete logarithm problem is hard, there exists a 4-round protocol that securely realizes the coin-tossing functionality with $1 /$ p-security.

We remark that if we allow our simulator to run in expected polynomial-time, we actually obtain perfect simulation against one of the parties and $1 / p$-security against the other (even against aborting adversaries). On the other hand, if we require strict polynomialtime simulation, where this polynomial is independent of the adversary's running time, our protocol achieves $1 / p$-security relative for both corruption cases. We further provide an abstraction for this protocol using a 2-round cryptographic primitive denoted by homomorphic trapdoor commitment scheme, where the commitment transcript, as well as the trapdoor, are homomorphic. This abstraction captures a larger class of hardness assumptions such as RSA and factoring.

Oblivious-Transfer and 2PC Next, we extend this idea to realize the oblivious-transfer functionality with $1 / p$-simulation security and privacy. In our first result, we construct an OT protocol that achieves $1 / p$-security and privacy against arbitrary (possibly aborting) malicious senders and full simulation security against non-aborting receivers. More precisely, an aborting receiver may violate the sender's privacy and learn its both inputs. We prove the following theorem:

Theorem 1.2. (Informal) Assuming the Decisional Diffie-Hellman (DDH) problem is hard, there exists a 4-round oblivious-transfer protocol, where the receiver receives the output at the end of the third round, which is $1 / p$-secure and private in the presence of aborting senders and fully secure in the presence of non-aborting receivers. ${ }^{2}$

It is important to note here that, if the receiver is required to learn the output only at the end of the fourth round, then the protocol of [55] already provides such a guarantee with full simulation security against malicious (aborting) senders and receivers. Our contribution is providing a protocol where the receiver learns the output in the third round. The main advantage of this protocol is that we can combine our oblivious-transfer protocol with the 2-round protocol of [43] to obtain 4-round secure computation where both parties receive the output with analogous security guarantees. Specifically, the receiver in the above OT protocol obtains its input already in the third round. This allows to apply the [43] protocol within the second and third OT rounds. More precisely, we obtain the following corollary:

\footnotetext{
${ }^{2}$ By fully secure, we mean standard simulation-based security.
} 
Theorem 1.3. (Informal) Assuming the DDH problem is hard, there exists a 4-round two-party secure protocol for any functionality, where both parties receive the output, that is $1 / p$-secure and private in the presence of aborting senders and fully secure in the presence of non-aborting receivers.

We remark that our protocol achieves a specific security level, namely $1 / p$-security and privacy against (arbitrary) senders and full security against non-aborting receivers. Our main motivation in this work is to understand what security is achievable in four rounds and Theorems 1.2 and 1.3 demonstrate that we can achieve security better than just privacy (as in [40]).

In our third protocol, we provide a different protocol for the oblivious-transfer functionality that guarantees $1 / p$-security against malicious (possibly aborting) senders while guaranteeing privacy against malicious (possibly aborting) senders and receivers based on claw-free trapdoor permutations. More formally, we obtain the following theorem.

Theorem 1.4. (Informal) Assuming the existence of claw-free permutations, there exists a 3-round oblivious-transfer protocol that is $1 /$ p-secure in the presence of aborting senders and private in the presence of aborting senders and receivers.

Comparing our two OT protocols, we note that they achieve incomparable notions of security with respect to malicious receivers. Specifically, the first protocol is fully secure in the presence of non-aborting adversaries and requires four rounds, whereas the second protocol requires only three rounds and achieves privacy against malicious (possibly aborting) receivers but no additional security if the receiver is non-aborting. Lower Bounds We complement our positive results with two lower bounds, where we show that achieving $1 / p$-security against aborting receivers is impossible under blackbox simulation. Our first result is:

Theorem 1.5. (Informal) Assuming NP $\nsubseteq B P P$, there exists no 3-round secure protocol for arbitrary functionalities with black-box simulation, with $1 / p$-security in the presence of malicious receivers and correctness with probability 1.

Our proof follows by extending the [28] lower bound, to show that 3-round black-box zero-knowledge proofs (or arguments) with negligible soundness and $1 / p$-security exist only for languages in BPP. Indeed, it is possible to construct zero-knowledge proofs with $1 / p$-soundness and $1 / p$-zero-knowledge security (for instance by repeating the Blum's Hamiltonicity proof [12] $\log p$ times).

Our second lower bound is:

Theorem 1.6. (Informal) There exists no 3-round oblivious-transfer protocol that achieves privacy in the presence of malicious senders and $1 / p$-security in the presence of malicious receivers for $p>2$. 
Table 1. Security guarantees with three and four rounds.

\begin{tabular}{lllll}
\hline & $\begin{array}{l}1 / p \text {-Sender w/ } \\
\text { Privacy for Both }\end{array}$ & $\begin{array}{l}\text { 1/p-Receiver } \\
\text { w/ Sender Privacy }\end{array}$ & $\begin{array}{l}1 / p \text {-Sender } \\
1 / p \text {-Receiver }\end{array}$ & $\begin{array}{l}1 / p \text {-Sender } \\
\text { Non-abort. Rec. }\end{array}$ \\
\hline 3-Rounds & [Thm 1.4] & BB impos. [Thm 1.5] & $?$ & $?$ \\
4-Rounds & Same as above & $?$ & Coin-tossing [Thm 1.1] & [Thm 1.3] \\
\hline
\end{tabular}

We remark that privacy against both parties is in some sense the minimal requirement of any secure computation protocol. Our lower bound shows that under this minimal requirement if we want to additionally achieve $1 / p$-security in three rounds, it can be achieved only against a malicious sender, which matches our upper bound, thus establishing its optimality (see Table 1).

\subsection{Our Techniques}

Toward understanding our relaxations and constructions, we begin with the impossibility result of Katz and Ostrovsky [45] of constructing 4-round two-party coin-tossing protocol via black-box simulation. On a high-level, given an arbitrary 4-round protocol for coin-tossing, the impossibility proceeds in two steps. In the first step, they consider an adversary $A_{1}$ that corrupts the party that receives the output first (in the third round) and aborts if the output belongs to some predetermined subset $\Gamma$. Next, using the simulator $S_{1}$ that exists for of $A_{1}$, they construct an adversary $A_{2}$ that corrupts the other party and biases the output to be in $\Gamma$. In particular, they prove that $A_{2}$ can force the output to be in $\Gamma$ with probability higher than what would occur in the ideal world. This contradicts the security of the coin-tossing protocol. Looking a bit more closely, for the proof to go through, they need to choose $\Gamma$ depending on the number of oracle queries made by the black-box simulator of $A_{1}$. Our first relaxation to circumvent this lower bound is to relax the simulation requirement to $1 / p$-indistinguishability. Now, we can argue that if $S_{1}$ makes more than $p$ queries to $A_{1}$ (which will be the case in our construction) then the bias induced by $A_{2}$ will be bounded by $1 / p$ and this will not contradict our security.

Moving on to more general protocol, we describe the difficulty in achieving three (or less) rounds protocols. Consider a three round protocol for oblivious-transfer. Since the receiver sends only one message (i.e., the second message), it would be impossible to rely on black-box techniques to extract the receiver's input. In fact, we show that it is impossible to achieve $1 / p$-simulation against malicious receivers. Therefore, for three rounds protocols, we relax the receiver's requirement to only privacy and demonstrate positive results that achieve privacy against malicious receivers and $1 / p$-simulation of malicious senders. Finally, we remark that another weakening of security that allows to circumvent the impossibility results is by restricting to non-aborting adversaries.

In what follows, we briefly sketch the technical details of our constructions beginning with our coin-tossing protocol.

Coin-Tossing (Section 3) We begin with a simple coin-tossing protocol. Party $P_{1}$ commits to a random string $s_{1}, P_{2}$ responds with random string $s_{2}$, followed by $P_{1}$ decommitting to $s_{1}$ to yield $s_{1} \oplus s_{2}$ as the result of the coin-tossing. Simulating such a 
coin-toss requires the commitment to have two properties: extraction and equivocation. Extraction will allow simulating a corrupted $P_{1}$ by first extracting $s_{1}$ and then setting $s_{2}=c \oplus s_{1}$ where $c$ is the coin-toss received from the ideal functionality. Equivocation, on the other hand, will allow simulating a corrupted $P_{2}$ by equivocating $s_{1}=c \oplus s_{2}$ in the decommitment. Therefore, the goal is to construct such a commitment scheme. Note that the extractable trapdoor commitment scheme from Pass and Wee [60] yields a 4-round commitment scheme resulting in a 6-round coin-tossing protocol with full security based on one-way permutations. In order to reduce the round complexity, one approach would be to run the last two-rounds of the our intuitive protocol in parallel with the 4-round commitment protocol; however, it is unclear how to demonstrate the security of such a protocol. Instead, we simply construct an equivocal commitment scheme based on the discrete logarithm assumption. In slight more detail, consider the following 4-round protocol based on the Pedersen's trapdoor commitment scheme [57]:

- $P_{1}$ samples $g, t_{0}, t_{1}$ and sends $g, h_{0}, h_{1}$ where $h_{i}=g^{t_{i}}$.

- $P_{2}$ sends $g^{\prime}=g^{s_{1}}\left(h_{0} h_{1}\right)^{r}$ for randomly chosen $r$ and $s_{1}$. Furthermore it sends a challenge bit $b$.

- $P_{1}$ sends $t_{b}$ and $s_{2}$.

- $P_{2}$ checks if $h_{b}=g^{t_{b}}$ and then reveals $s_{1}$ and $r$.

Finally, $P_{1}$ checks if $g^{\prime}=g^{s_{1}}\left(h_{0} h_{1}\right)^{r}$ and outputs $s_{1} \oplus s_{2}$ as the result of the coin-toss.

We first demonstrate security against non-aborting adversaries. Simulating a corrupted $P_{1}$ involves extracting $t_{0}$ and $t_{1}$ by rewinding and then equivocating $g^{\prime}$ to any $\widehat{s}_{1}$ by setting $\widehat{r}=\left(s_{1}-\widehat{s_{1}}\right) /\left(t_{0}+t_{1}\right)+r \bmod p$ and revealing $\widehat{s_{1}}, \widehat{r}$. Indistinguishability follows from perfect hiding property of the Pedersen commitment. Simulating a corrupted $P_{2}$ involves obtaining $s_{1}$ from the last message and then rewinding to set $s_{2}=c-s_{1} \bmod p$. Security here follows from the fact that if $P_{2}$ can equivocate, then we can use $P_{2}$ to extract the discrete logarithm of $h_{1-b}$ w.r.t. $g$.

This simple protocol can, in fact, achieve $1 / p$-security against a corrupted (aborting) $P_{2}$ by slightly modifying the simulator to rewind $P_{2} n p$ times to extract $s_{1}$. On a high level, security can be argued by considering two cases. If $P_{2}$ aborts with probability higher than $1-1 / p$, then the simulator can simply output an aborting views which already achieves $1 / p$-indistinguishability. Otherwise, if it aborts with probability smaller than $1-1 / p$, then rewinding will succeed except with negligible probability and we can perfectly simulate $P_{2}$ 's view (conditioned on extracting $s_{1}$ ).

Our final protocol upgrades the security of this basic protocol to achieve full security against $P_{1}$ via parallel repetition. Instead of having one pair of generators $h_{0}, h_{1}$, we will have $P_{1}$ send $n$ pairs $\left\{\left(h_{0}^{i}, h_{1}^{i}\right)\right\}_{i \in[n]}$ and require $P_{2}$ to commit to its input as $g^{m}\left(h_{0}^{1} h_{1}^{1}\right)^{r_{1}}\left(h_{0}^{2} h_{1}^{2}\right)^{r_{2}} \ldots\left(h_{0}^{n} h_{1}^{n}\right)^{r_{n}}$ and send an $n$-bit challenge $e_{1}, \ldots, e_{n} . P_{1}$ responds with $s_{2}$ and $\log _{g}\left(h_{e_{i}}^{i}\right)$ for $i \in[n]$ followed by $P_{2}$ decommitting to $s_{1}$. The reason we get full security against a corrupted $P_{1}$ is because we can with very high probability extract both $t_{0}^{i}$ and $t_{1}^{i}$ for some $i$ by rewinding.

\section{4-Round 2PC Against Non-aborting Adversaries (Section 4) Our first OT protocol} employs a common paradigm for securely realizing this functionality. Namely, the receiver picks two public keys for which it knows only one of the corresponding secret keys and sends them to the sender that uses these keys to encrypt its OT inputs. If indeed 
the receiver knows only one of the secret keys, then it will not be able to decrypt both inputs. In slight more detail, a basic version of our protocol proceeds as follows:

- $S$ samples $g, t_{0}, t_{1}$ and sends $h_{i}=g^{t_{i}}$.

- $R$ with input $b$ computes $\mathrm{PK}_{b}=g^{m}$ and $\mathrm{PK}_{1-b}=\left(h_{0} h_{1}\right)^{\tilde{m}}$ for randomly chosen $m, \tilde{m}$.

- $S$ with input $s_{0}, s_{1}$ encrypts $s_{0}$ and $s_{1}$ with public keys $\mathrm{PK}_{0}$ and $\mathrm{PK}_{1}$ using the El Gamal encryption scheme.

- $R$ decrypts $\mathrm{PK}_{b}$ using $\mathrm{SK}_{b}=m$.

This protocol is essentially secure against a malicious sender as the receiver's message information theoretically hides its bit $b$. The main challenge to make this secure in the presence of a malicious receiver is in designing a mechanism to enforce the receiver to choose its public keys correctly. In the preceding protocol, the receiver is asked to send a public key for the unknown secret key to take a particular form, for which the receiver does not know the trapdoor associated with it (concretely, this trapdoor is a discrete logarithm of a generator picked by the sender). We can enforce such a requirement by including a witness-indistinguishable proof-of-knowledge (WI-PoK) from the receiver. This further will allow to extract the bit $b$ for which the receiver indeed knows the corresponding secret key (which implies input extraction of the receiver's input).

Slightly more formally, to argue security against non-aborting parties we observe that we can extract $t_{0}$ and $t_{1}$ from a non-aborting sender. This will allow us to equivocate the receiver's input and learn both $s_{0}, s_{1}$. To simulate a non-aborting receiver, we extract the bit $b$ from the PoK by rewinding and then simulate.

Finally, to obtain secure computation for general functionalities, we combine our OT with the 2-round protocol of [43] which is specified in the OT-hybrid model. This protocol provides an output to only one of the parties (namely, the receiver of the OT instances). Yet, we run this protocol in parallel with our OT protocol where the second and third messages of the OT protocol run in parallel with the [43] protocol. As a result, the receiver of the OT receives the output of the computation at the end of third round. Finally, to extend this protocol to have outputs delivered to both parties, we can rely on the fourth round where the receiver transmits the output to the sender.

\section{4-Round 2PC with 1/p-Security Against Aborting Senders and Full Security Against} Non-aborting Receivers (Section 5)

Next, we upgrade the security of our OT to handle aborting adversaries. We begin with the observation that our previous OT protocol is already $1 / p$-secure for $p=1+\frac{1}{3}$ against malicious aborting senders. To see this, suppose that for some trapdoor the sender aborts with probability at most $\frac{1}{2}$, then in expectation the simulator needs to rewind the sender just twice in order to extract this trapdoor. If both trapdoors satisfy this condition, then the simulator can easily extract both of them. Now, suppose that the sender aborts with probability at least $\frac{1}{2}$ when it is asked to open one of the trapdoors. Then, the overall probability with which the sender aborts is $\frac{1}{4}$ (as each trapdoor is requested to be revealed with probability $\frac{1}{2}$ ). In order to achieve $\frac{3}{4}$ security, it suffices to output a distribution that is $\frac{3}{4}$-close to the real distribution. As the sender aborts with probability 
at least $\frac{1}{4}$ a simulator that simply outputs all the views on which the sender aborts already achieves $\frac{3}{4}$ security.

With this observation, we show that $1 / p$-security for an arbitrary polynomial $p$ can be achieved by amplifying the indistinguishability argument via parallel repetition. More precisely, by repeating the basic protocol $O(\kappa p)$ times, where $\kappa$ is the security parameter, we can show, using a careful application of Yao-type amplification [62], that if the adversary does not abort with probability at least $\Omega(1 / p)$, then the simulation can extract most of the trapdoors. This idea is used in conjunction with the combiner of Ostrovsky, Richelson and Scafuro [55] to ensure that the simulator extracts the sender's inputs if and only if the receiver successfully extracts it, or in other words, prevents any form of input dependent attacks. As in the previous construction, the current OT is also private in the presence of aborting senders.

\section{3-Round OT with 1/p-Security Against Aborting Senders and Privacy Against Aborting} Receivers (Section 6) We conclude with our third OT protocol which demonstrates the feasibility of $1 / p$ sender security and privacy against aborting receivers in three rounds. We begin with a basic protocol that only achieves privacy and then amplify it security to get $1 / p$ sender simulation. Our protocol is based on claw-free trapdoor permutations. Namely, the sender samples a pair of functions $f_{0}, f_{1}$ from a claw-free family and provides the description to the receiver. The receiver then samples $y=f_{b}(x)$ for a random $x$ and returns $y$ to the sender. Finally, the sender uses the trapdoors for $f_{0}$ and $f_{1}$ to obtain $x_{b}=f_{b}^{-1}(x)$ and masks its inputs $\left(s_{0}, s_{1}\right)$ with the Goldreich-Levin hardcore predicate of $x_{b}$. To prove receiver privacy, we need to show it is impossible for the receiver to distinguish both the games where the sender's input are sampled according to $\left(s_{0}, U\right)$ and $\left(U, s_{1}\right)$ from the real-game (where $U$ is the uniform distribution over $\{0,1\}$ ). We argue that if such a receiver exists, then using the list-decodable extractor guaranteed by the Goldreich-Levin Theorem we can extract $x_{0}$ and $x_{1}$, thus finding a claw, i.e., $x_{0}$ and $x_{1}$ such that $f_{0}\left(x_{0}\right)=f_{1}\left(x_{1}\right)$. This reduction is subtle and requires using a careful averaging argument. We next amplify this protocol to achieve $1 / p$ sender simulation based on a similar amplifying technique as in the previous construction. Our protocol can be implemented based on the RSA claw-free collection of functions.

\subsection{Subsequent Work}

Following our work, the area of round complexity has become an active area of research. We summarize briefly the results that have appeared since our publication. In the twoparty and non-simultaneous message model, Ciampi et al. [20] showed how to obtain a 4-round protocol based on trapdoor permutations. Garg et al. [32] studied the exact round complexity of multiparty computation in the simultaneous message model. In the case of semi-honest adversaries (or even the slightly stronger setting of semi-malicious ${ }^{3}$ adversaries), three round protocols based on the Learning With Errors assumption were constructed by Brakerski et al. [9]. Ananth et al. [1] gave a 5-round multiparty protocol against malicious adversaries based on DDH. Under sub-exponential hardness assump-

\footnotetext{
${ }^{3} \mathrm{~A}$ semi-malicious adversary is allowed to invoke a corrupted party with arbitrary chosen input and random tape, but otherwise follows the protocol specification honestly as a passive adversary.
} 
tions, 4-round constructions were demonstrated in [1,9]. Under some relaxations of super-polynomial simulation, the work of Badrinarayanan et al. [7] showed how to obtain 3-round concurrently secure multi-party protocol assuming subexponentially secure LWE and DDH. For specific multi-party functionalities, 4-round constructions have been obtained, e.g., coin-tossing by Ciampi et al. [20]. In two recent works, Benhamouda and Lin [11] and Garg and Srinivasan [36], the round complexity of multiparty computation in the semi-honest model was completely resolved, where they provide a 2-round constructions (based on 2-round OT). Additionally, the work of [11] provided a 5-round multi-party protocol against malicious adversaries-based standard assumptions. Finally, Halevi et al. [38] and Badrinarayanan et al. [8] designed the first 4-round multi-party protocol (i.e., round-optimal) based on standard polynomial-time hardness assumptions and black-box simulation in the plain model. Interestingly, the work of [8] introduces the notion of promise-ZK which essentially considers security in the presence of nonaborting adversaries as a stepping stone toward achieving full security.

\section{Preliminaries}

\subsection{Basic Notations}

We denote the security parameter by $n$. We say that a function $\mu: \mathbb{N} \rightarrow \mathbb{N}$ is negligible if for every positive polynomial $p(\cdot)$ and all sufficiently large $n$ it holds that $\mu(n)<\frac{1}{p(n)}$. We use the abbreviation PPT to denote probabilistic polynomial-time. We further denote by $a \leftarrow A$ the random sampling of $a$ from a distribution $A$, and by $[n]$ the set of elements $\{1, \ldots, n\}$.

Computational Indistinguishability We specify the definitions of computational indistinguishability and computational $\frac{1}{p}$-indistinguishability.

Definition 2.1. Let $X=\{X(a, n)\}_{a \in\{0,1\}^{*}, n \in \mathbb{N}}$ and $Y=\{Y(a, n)\}_{a \in\{0,1\}^{*}, n \in \mathbb{N}}$ be two distribution ensembles. We say that $X$ and $Y$ are computationally indistinguishable, denoted $X \stackrel{\mathrm{c}}{\approx} Y$, if for every PPT distinguisher $D$ there exists a negligible function $\mu(\cdot)$ such that for every $a \in\{0,1\}^{*}$ and all sufficiently large $n$

$$
\left|\operatorname{Pr}\left[D\left(X(a, n), 1^{n}\right)=1\right]-\operatorname{Pr}\left[D\left(Y(a, n), 1^{n}\right)=1\right]\right|<\mu(n) .
$$

Definition 2.2. Let $X=\{X(a, n)\}_{a \in\{0,1\}^{*}, n \in \mathbb{N}}$ and $Y=\{Y(a, n)\}_{a \in\{0,1\}^{*}, n \in \mathbb{N}}$ be two distribution ensembles. We say that $X$ and $Y$ are computationally $1 / p$-indistinguishable, denoted $X \stackrel{1 / \mathrm{p}}{\approx} Y$, if for every PPT distinguisher $D$ there exists a negligible function $\mu(\cdot)$ such that for every $a \in\{0,1\}^{*}$ and all sufficiently large $n$

$$
\left|\operatorname{Pr}\left[D\left(X(a, n), 1^{n}\right)=1\right]-\operatorname{Pr}\left[D\left(Y(a, n), 1^{n}\right)=1\right]\right|<\frac{1}{p(n)}+\mu(n) .
$$

Statistical Distance Next we specify the distance measure of statistical closeness. 
Definition 2.3. Let $X_{n}$ and $Y_{n}$ be random variables accepting values taken from a finite domain $\Omega \subseteq\{0,1\}^{n}$. The statistical distance between $X_{n}$ and $Y_{n}$ is

$$
S D\left(X_{n}, Y_{n}\right)=\frac{1}{2} \sum_{\omega \in \Omega}\left|\operatorname{Pr}\left[X_{n}=\omega\right]-\operatorname{Pr}\left[Y_{n}=\omega\right]\right|
$$

We say that $X_{n}$ and $Y_{n}$ are $\varepsilon$-close if their statistical distance is at most $S D\left(X_{n}, Y_{n}\right) \leq$ $\varepsilon(n)$. We say that $X_{n}$ and $Y_{n}$ are statistically close, denoted $X_{n} \approx_{s} Y_{n}$, if $\varepsilon(n)$ is negligible in $n$.

\subsection{Hardness Assumptions}

Our constructions rely on the following hardness assumptions.

Discrete Logarithm The classic discrete logarithm assumption is stated as follows.

Definition 2.4. (DL) We say that the discrete logarithm $(D L)$ problem is hard relative to $\mathcal{G}$, if for any PPT adversary $A$ there exists a negligible function negl such that

$$
\operatorname{Pr}\left[x \leftarrow A\left(\mathbb{G}, p, g, g^{x}\right)\right] \leq \operatorname{negl}(n),
$$

where $(\mathbb{G}, p, g) \leftarrow \mathcal{G}\left(1^{n}\right)$ and the probability is taken over the choice of $x \leftarrow \mathbb{Z}_{q}$.

Decisional Diffie-Hellman The decisional Diffie-Hellman assumption is stated as follows.

Definition 2.5. (DDH) We say that the decisional Diffie-Hellman (DDH) problem is hard relative to $\mathcal{G}$, if for any PPT distinguisher $D$ there exists a negligible function negl such that

$$
\left|\operatorname{Pr}\left[D\left(\mathbb{G}, p, g, g^{x}, g^{y}, g^{z}\right)=1\right]-\operatorname{Pr}\left[D\left(\mathbb{G}, p, g, g^{x}, g^{y}, g^{x y}\right)=1\right]\right| \leq \operatorname{negl}(n),
$$

where $(\mathbb{G}, p, g) \leftarrow \mathcal{G}\left(1^{n}\right)$ and the probabilities are taken over the choices of $x, y, z \leftarrow$ $\mathbb{Z}_{q}$.

\subsection{Commitment Schemes}

Statistically hiding commitment schemes maintains two important security properties of hiding and biding, where the flavor of the hiding property is statistical. More formally,

Definition 2.6. A commitment scheme is a pair of probabilistic polynomial-time algorithms, denoted (Sen, Rec) (for sender and receiver), satisfying the following:

- Inputs The common input is a security parameter $1^{n}$. The sender has a secret input $m \in \mathcal{M}_{n}$ where $\mathcal{M}_{n}$ is the message space of the sender's input. 
- Hiding For every probabilistic polynomial-time algorithms Rec* interacting with Sen and every two messages $m, m^{\prime} \in \mathcal{M}_{n}$, the random variables describing the output of $\operatorname{Rec}^{*}$ in the two cases, namely $\left\langle\operatorname{Sen}(m), \operatorname{Rec}^{*}\right\rangle\left(1^{n}\right)$ and $\left\langle\operatorname{Sen}\left(m^{\prime}\right), \operatorname{Rec}^{*}\right\rangle\left(1^{n}\right)$, are statistically close.

- Binding A receiver's view of an interaction with the sender, denoted $(r, \bar{m})$, consists of the random coins used by the receiver (namely, $r$ ) and the sequence of messages received from the receiver (namely, $\bar{m}$ ).

Let $m, m^{\prime} \in \mathcal{M}_{n}$. We say that the receiver's view (of such interaction), $(r, \bar{m})$, is a possible $m$-commitment if there exists a string $s$ such that $\bar{m}$ describes the messages received by Rec when Rec uses local coins $r$ and interacts with Sen which uses local coins $s$ and has input $\left(1^{n}, m\right)$. We denote $\bar{m}$ by $\mathbf{V i e w}\langle\operatorname{Sen}(m), \operatorname{Rec}\rangle\left(1^{n}\right)$.

We say that the receiver's view $(r, \bar{m})$ is ambiguous if is it both a possible $m$-commitment and a possible $m^{\prime}$-commitment.

The binding property asserts that, for all but a negligible fraction of the coins toss of the receiver, there exists no sequence of messages (from the sender) which together with these coin toss forms an ambiguous receiver view. Namely, that for all but a negligible function of the $r \in\{0,1\}^{\text {polyn }}$ there is no $\bar{m}$ such that $(r, \bar{m})$ is ambiguous.

\subsubsection{Trapdoor Commitment Schemes}

Loosely speaking, a trapdoor commitment scheme is a commitment scheme that meets the classic binding and hiding security properties specified in Definition 2.6, yet it allows to decommit a commitment into any value from the message space given some trapdoor information. In this paper, we view the commit phase of the trapdoor commitment schemes as a 2-round protocol $\pi_{\mathrm{COM}}=\left(\pi_{\mathrm{Rec}}, \pi_{\mathrm{Sen}}\right)$ where the receiver sends the first message $\pi_{\text {Rec }}$ and the sender responds with message $\pi_{\text {Sen }}$ (that is, in a real execution the receiver knows the trapdoor, whereas in the simulation the simulator extracts this trapdoor from the receiver in order to equivocate its commitment). Formally stating,

Definition 2.7. A 2-round trapdoor commitment scheme is a pair of probabilistic polynomial-time algorithms, denoted (Sen, Rec) (for sender and receiver), satisfying the following:

- Inputs The common input is a security parameter $1^{n}$. The sender has a secret input $m \in \mathcal{M}_{n}$.

- (Sen, Rec) is a commitment scheme in the sense of Definition 2.6 with perfect hiding.

- For any probabilistic polynomial-time algorithm Rec*, there exists a polynomialtime algorithm $\mathcal{S}=\left(\mathcal{S}_{1}, \mathcal{S}_{2}\right)$ such that for any sequence of messages $\left\{m_{n}\right\}_{n \in \mathbb{N}}$ where $m_{n} \in \mathcal{M}_{n}$ for all $n$, the following holds:

On input $1^{n}$ simulator $\mathcal{S}_{1}$ (playing the receiver) outputs $\pi_{\operatorname{Rec}}$ and a trapdoor td. Simulator $\mathcal{S}_{2}$ is defined as follows:

- First, on input $1^{n}$ and randomness $R, \mathcal{S}_{2}$ outputs $\pi_{\operatorname{Sen}}^{\mathcal{S}_{2}}$ in response to $\pi_{\operatorname{Rec}}$ such that the distributions of $\left\{\pi_{\mathrm{Sen}}^{\mathcal{S}_{2}}\right\}_{n \in \mathbb{N}}$ and $\left\{\pi_{\mathrm{Sen}}\right\}_{n \in \mathbb{N}}$ are identical.

- Next, on input td, message $m_{n}$ and randomness $R$, simulator $\mathcal{S}_{2}$ outputs coins $s$ such that $\pi_{\text {Sen }}^{\mathcal{S}}=\operatorname{Sen}\left(1^{n}, \pi_{\operatorname{Rec}}, m_{n} ; s\right)$. 
Homomorphic Trapdoor Commitment Schemes We consider trapdoor commitments that are homomorphic in the sense that given two receiver's messages $\pi_{\operatorname{Rec}}^{1}$ and $\pi_{\operatorname{Rec}}^{2}$ that are defined relative to some group $\mathbb{G}$, it is possible to combine them into a single receiver's message $\pi_{\operatorname{Rec}}=\pi_{\operatorname{Rec}}^{1} \cdot \pi_{\operatorname{Rec}}^{2}$. Moreover, the trapdoor can be homomorphically updated as well. One such example is Pedersen's commitment scheme that is based on the hardness of Discrete logarithm [57]. Loosely speaking, given a group description $\mathbb{G}$ of prime order $q$, and two generators $g, h$, a commitment of $m \in \mathbb{Z}_{q}$ is computed by $c=g^{m} h^{r}$ for a random $r \leftarrow \mathbb{Z}_{q}$. Moreover, the knowledge of $\log _{g} h$ enables to open $c$ into any message in $\mathbb{Z}_{q}$. Note that given two generators $h_{0}$ and $h_{1}$ one can assemble a new generator $h_{0} h_{1}$ for which the trapdoor will be $\log _{g} h_{0}+\log _{g} h_{1}$.

Two additional trapdoor commitment schemes that fit into our framework are numbertheoretic-based constructions in composite order groups. Concretely, we consider two constructions in $\mathbb{Z}_{N}^{*}$ for RSA composite $N$ with security based on the RSA and factoring hardness assumptions. The trapdoor information of these constructions does not require the knowledge of the factorization of $N$; thus, $N$ can be part of the group description handed to the parties at the onset of the protocol (similarly to the group description $\mathbb{G}$ in the prior example). Loosely speaking, a commitment to a message $m \in \mathbb{Z}_{e}$ in the RSAbased construction is computed by $g^{m} r^{e} \bmod N$, where $r$ is picked at random from $\mathbb{Z}_{N}^{*}$, $g=x^{e} \bmod N$ and $(N, e)$ can be considered as the public parameters (such that $e$ is relatively prime to $\varphi(N))$. Moreover, the trapdoor picked by the receiver is $x$. Clearly, given $g_{1}=x_{1}^{e} \bmod N$ and $g_{2}=x_{2}^{e} \bmod N$, then $g_{1} g_{2}=\left(x_{1} x_{2}\right)^{e} \bmod N$.

An additional factoring-based trapdoor construction implies a commitment to a message $m \in \mathbb{Z}_{2^{t}}$ by $g^{m} r^{2^{\tau+t}} \bmod N$ for a random $r$, such that $g=x^{2^{\tau+t}} \bmod N$ and $(N, \tau, t)$ can be considered as the public parameters. Moreover, the trapdoor picked by the receiver is $x$. The detailed descriptions of these commitment schemes can be found in [24].

\subsection{Witness Indistinguishability}

A proof system between a prove and a verifier is witness indistinguishable if the proof does not leak information about which witness the prover is using, even if the verifier is malicious. In the following, we let $\langle\mathcal{P}(y), \mathcal{V}(z)(x)\rangle$ denote the view of verifier $\mathcal{V}$ when interacting with prover $\mathcal{P}$ on common input $x$, when $\mathcal{P}$ has auxiliary input $y$ and $\mathcal{V}$ has auxiliary input $z$.

Definition 2.8. [26] Let $L \in N P$ and let $(\mathcal{P}, \mathcal{V})$ be an interactive proof system for $L$ with perfect completeness. We say that $(\mathcal{P}, \mathcal{V})$ is witness-indistinguishable (WI) if for every PPT algorithm $\mathcal{V}^{*}$ and every two sequences $\left\{w_{x}^{1}\right\}_{x \in L}$ and $\left\{w_{x}^{2}\right\}_{x \in L}$ such that $w_{x}^{1}$ and $w_{x}^{2}$ are both witnesses for $x \in L$, the following ensembles are computationally indistinguishable:

1. $\left\{\left\langle\mathcal{P}\left(w_{x}^{1}\right), \mathcal{V}(z)\right\rangle(x)\right\}_{x \in L, z \in\{0,1\}}$.

2. $\left\{\left\langle\mathcal{P}\left(w_{x}^{2}\right), \mathcal{V}(z)\right\rangle(x)\right\}_{x \in L, z \in\{0,1\}}$. 


\subsection{Secret Sharing}

A secret-sharing scheme allows distribution of a secret among a group of $n$ players, each of whom in a sharing phase receive a share (or piece) of the secret. In its simplest form, the goal of secret sharing is to allow only subsets of players of size at least $t+1$ to reconstruct the secret. More formally a $t+1$-out-of- $n$ secret-sharing scheme comes with a sharing algorithm that on input a secret $s$ outputs $n$ shares $s_{1}, \ldots, s_{n}$ and a reconstruction algorithm that takes as input $\left(s_{i}\right)_{i \in S}, S$ where $|S|>t$ and outputs either a secret $s^{\prime}$ or $\perp$. In this work, we will use the Shamir's secret-sharing scheme [61] with secrets in $\mathbb{F}=G F\left(2^{n}\right)$. We present the sharing and reconstruction algorithms below:

Sharing Algorithm For any input $s \in \mathbb{F}$, pick a random polynomial $f(\cdot)$ of degree $t$ in the polynomial-field $\mathbb{F}[x]$ with the condition that $f(0)=s$ and output $f(1), \ldots, f(n)$.

Reconstruction Algorithm For any input $\left(s_{i}^{\prime}\right)_{i \in S}$ where none of the $s_{i}^{\prime}$ are $\perp$ and $|S|>t$, compute a polynomial $g(x)$ such that $g(i)=s_{i}^{\prime}$ for every $i \in S$. This is possible using Lagrange interpolation where $g$ is given by

$$
g(x)=\sum_{i \in S} s_{i}^{\prime} \prod_{j \in S /\{i\}} \frac{x-j}{i-j} .
$$

Finally the reconstruction algorithm outputs $g(0)$.

We will additionally rely on a property of this secret-sharing scheme that has been observed by Ostrovsky, Richelson and Scafuro in [55]. Toward that, we view the Shamir secret-sharing scheme as a linear code generated by the following $n \times(t+1)$ Vandermonde matrix

$$
A=\left(\begin{array}{cccc}
1 & 1^{2} & \cdots & 1^{t} \\
1 & 2^{2} & \cdots & 2^{t} \\
\vdots & \vdots & \vdots & \vdots \\
1 & n^{2} & \cdots & n^{t}
\end{array}\right)
$$

More formally, the shares of a secret $s$ that are obtained via a polynomial $f$ in the Shamir scheme can be obtained by computing $A \mathbf{c}$ where $\mathbf{c}$ is the vector containing the coefficients of $f$. Next, we recall that for any linear code $A$, there exists a parity check matrix $H$ of dimension $(n-t-1) \times n$ which satisfies the equation $H A=\mathbf{0}_{(n-t-1) \times(t+1)}$, i.e., the all 0 's matrix. We thus define the linear operator $\phi(v)=H v$ for any vector $v$. Then it holds that any set of shares $\mathbf{S}$ is valid if and only if it satisfies the equation $\phi(\mathbf{s})=\mathbf{0}_{n-t-1}$.

\subsection{Hardcore Predicates}

Definition 2.9. (Hardcore predicate) Let $f:\{0,1\}^{n} \rightarrow\{0,1\}^{*}$ and $\mathrm{H}:\{0,1\}^{n} \rightarrow$ $\{0,1\}$ be a polynomial-time computable functions. We say $\mathrm{H}$ is a hardcore predicate of $f$, if for every PPT machine $A$, there exists a negligible function negl $(\cdot)$ such that

$$
\operatorname{Pr}\left[x \leftarrow\{0,1\}^{n} ; y=f(x): A\left(1^{n}, y\right)=\mathrm{H}(x)\right] \leq \frac{1}{2}+\operatorname{negl}(n) .
$$


An important theorem by Goldreich and Levin [31] states that if $f$ is a one-way function over $\{0,1\}^{n}$ then the one-way function $f^{\prime}$ over $\{0,1\}^{2 n}$, defined by $f^{\prime}(x, r)=$ $(f(x), r)$, admits the following hardcore predicate $b(x, r)=\langle x, r\rangle=\Sigma x_{i} r_{i} \bmod 2$, where $x_{i}, r_{i}$ is the $i$ th bit of $x, r$ respectively. In the following, we refer to this predicate as the GL bit of $f$. We will use the following theorem that establishes the list-decoding property of the GL bit.

Theorem 2.10. ([31]) There exists a PPT oracle machine Inv that on input $(n, \varepsilon)$ and oracle access to a predictor PPT $B$, runs in time poly $\left(n, \frac{1}{\varepsilon}\right)$, makes at most $O\left(\frac{n^{2}}{\varepsilon^{2}}\right)$ queries to $B$ and outputs a list $L$ with $|L| \leq \frac{4 n}{\varepsilon^{2}}$ such that if

$$
\operatorname{Pr}\left[r \leftarrow\{0,1\}^{n}: B(r)=\langle x, r\rangle\right] \geq \frac{1}{2}+\frac{\varepsilon}{2}
$$

then

$$
\operatorname{Pr}\left[L \leftarrow \operatorname{lnv}^{B}(n, \varepsilon): x \in L\right] \geq \frac{1}{2} .
$$

\subsection{Oblivious-Transfer}

In this section, we consider an extension of the privacy definition in the presence of malicious receivers and senders shown in [40]. In the definitions presented below, we denote the honest sender and receiver algorithms by Sen and Rec respectively. Recall that the oblivious-transfer functionality is specified by the function. $\mathcal{F}_{\text {От }}:\left(\left(s_{0}, s_{1}\right), b\right) \mapsto$ $\left(-, s_{b}\right)$ that takes as input $\left(s_{0}, s_{1}\right)$ from the sender a bit $b$ from the receiver. We say that a protocol $\pi=\langle\operatorname{Sen}, \operatorname{Rec}\rangle$ realizes the OT functionality if the protocol computes $\mathcal{F}_{\text {OT }}$ correctly.

We will let View $\mathcal{A}\left[\mathcal{A}\left(1^{n}\right), \operatorname{Rec}\left(1^{n}, b\right)\right]$ is the r.v. representing the view of the adversary $\mathcal{A}$ when interacting with $\operatorname{Rec}\left(1^{n}, b\right)$ and $\operatorname{View}_{\mathcal{A}}\left[\operatorname{Sen}\left(1^{n},\left(s_{0}, s_{1}\right)\right), \mathcal{A}\left(1^{n}\right)\right]$ is the r.v. representing the view of the adversary $\mathcal{A}$ when interacting with $\operatorname{Sen}\left(1^{n},\left(s_{0}, s_{1}\right)\right)$. First, we recall the definition of defensibly private oblivious-transfer and then proceed to our definition.

\subsubsection{Defensibly Private Oblivious-Transfer}

The notion of defensible privacy was introduced by Haitner in [37,39]. A defense in a two-party protocol $\pi=\left(P_{1}, P_{2}\right)$ execution is an input and random tape provided by the adversary after the execution concludes. A defense for a party controlled by the adversary is said to be good, if this party participated honestly in the protocol using this very input and random tape, then it would have resulted in the exact same messages that were sent by the adversary. In essence, this defense serves as a proof of honest behavior. It could very well be the case that an adversary deviates from the protocol in the execution but later provides a good defense. The notion of defensible privacy says that a protocol is private in the presence of defensible adversaries if the adversary learns nothing more than its prescribed output when it provides a good defense. 
We begin with informally describing the notion of good defense for a protocol $\pi$; we refer to [39] for the formal definition. Let trans $=\left(q_{1}, a_{1}, \ldots, q_{\ell}, a_{\ell}\right)$ be the transcript of an execution of a protocol $\pi$ that is engaged between $P_{1}$ and $P_{2}$ and let $\mathcal{A}$ denote an adversary that controls $P_{1}$, where $q_{i}$ is the $i$ th message from $P_{1}$ and $a_{i}$ is the $i$ th message from $P_{2}$ (that is, $a_{i}$ is the response for $q_{i}$ ). Then we say that $(x, r)$ constitutes a good defense of $\mathcal{A}$ relative to trans if the transcript generated by running the honest algorithm for $P_{1}$ with input $x$ and random tape $r$ against $P_{2}$ 's messages $a_{1}, \ldots, a_{\ell}$ results exactly in trans.

The notion of defensible privacy can be defined for any secure computation protocol. Nevertheless, since we are only interested in oblivious-transfer protocols, we present a definition below that is restricted to oblivious-transfer protocols. The more general definition can be found in [39]. At a high-level, an OT protocol is defensibly private with respect to a corrupted sender if no adversary interacting with an honest receiver with input $b$ should be able to learn $b$, if at the end of the execution the adversary produces any good defense. Similarly, an OT protocol that is defensibly private with respect to malicious receivers requires that any adversary interacting with an honest sender with input $\left(s_{0}, s_{1}\right)$ should not be able to learn $s_{1-b}$, if at the end of the execution the adversary produces a good defense with input $b$. Below we present a variant of the definition presented in [39]. We stress that while the [39] definition only considers bit OT (i.e., sender's inputs are bits) we consider string OT.

Definition 2.11. (Defensible-Private String OT) Let $\pi=\langle\operatorname{Sen}, \mathrm{Rec}\rangle$ be a two-party protocol that between a sender Sen and a receiver Rec that realizes the OT functionality. Malicious Sender We say that $\pi$ is a defensibly private string oblivious-transfer protocol w.r.t a malicious sender, if for every PPT adversary $\mathcal{A}$ the following holds,

$$
\left\{\Gamma\left(\operatorname{View}_{\mathcal{A}}\left[\mathcal{A}\left(1^{n}\right), \operatorname{Rec}\left(1^{n}, U\right)\right], U\right)\right\} \stackrel{\mathrm{c}}{\approx}\left\{\Gamma\left(\operatorname{View}_{\mathcal{A}}\left[\mathcal{A}\left(1^{n}\right), \operatorname{Rec}\left(1^{n}, U\right)\right], U^{\prime}\right)\right\}
$$

where $\Gamma$ is the function that on input $(v, \sigma)$ outputs $(v, \sigma)$ if following the execution $\mathcal{A}$ outputs a good defense for $\pi$, and $\perp$ otherwise, and $U$ and $U^{\prime}$ are independent random variables uniformly distributed over $\{0,1\}$.

Malicious Receiver We say that $\pi$ is a defensibly private string oblivious-transfer protocol w.r.t a malicious receiver, if for every PPT adversary $\mathcal{A}$ the following holds,

$$
\begin{aligned}
& \left\{\Gamma\left(\operatorname{View}_{\mathcal{A}}\left[\operatorname{Sen}\left(1^{n},\left(U_{0}^{n}, U_{1}^{n}\right)\right), \mathcal{A}\left(1^{n}\right)\right], U_{1-b}^{n}\right)\right\} \\
& \quad \stackrel{\mathrm{c}}{\approx}\left\{\Gamma\left(\operatorname{View}_{\mathcal{A}}\left[\operatorname{Sen}\left(1^{n},\left(U_{0}^{n}, U_{1}^{n}\right)\right), \mathcal{A}\left(1^{n}\right)\right], \bar{U}^{n}\right)\right\}
\end{aligned}
$$

where $\Gamma$ is the function that on input $(v, \sigma)$ outputs $(v, \sigma)$ if following the execution $\mathcal{A}$ outputs a good defense for $\pi$, and $\perp$; otherwise, $b$ is the Rec's input in this defense and $U_{0}^{n}, U_{1}^{n}, \bar{U}^{n}$ are independent random variables uniformly distributed over $\{0,1\}^{n}$.

\subsubsection{Private Oblivious-Transfer}

In this section, we present our definition. We will focus on the case of a 3-round protocol where the sender sends the first message. Furthermore, our definition will restrict the 
honest sender's algorithm to be described by a pair of algorithms $\operatorname{Sen}=\left(\operatorname{Sen}_{1}, \operatorname{Sen}_{2}\right)$ where $\operatorname{Sen}_{1}$ on input $1^{n}$ outputs the first message $m_{s}^{1}$ of the OT protocol and state $\sigma$ and Sen$_{2}$ on input $\left(\sigma, m_{r},\left(s_{0}, s_{1}\right)\right)$ generates the third message of the OT protocol where $m_{r}$ is the response from the receiver and $\left(s_{0}, s_{1}\right)$ is the sender's input.

Then, for a malicious receiver $\operatorname{Rec}^{*}$ let $\operatorname{PExec}_{\operatorname{Rec}^{*}}\left(1^{n}\right)=\left((a, q), \sigma, r_{\operatorname{Rec}}\right)$ be the r.v. where $\left(m_{s}^{1}, m_{r}\right)$ represent the first two messages exchanged in the interaction between $\operatorname{Sen}_{1}$ on input $1^{n}$ and a receiver $\operatorname{Rec}^{*}$ with input $1^{n}$ and randomness $r_{\operatorname{Rec}}$ and $\sigma$ is the state output by $\operatorname{Sen}_{1}$. We now define privacy against a malicious receiver as follows. In essence, PExec represents the state of the execution after the first two messages have been exchanged.

Definition 2.12. (Sender's Privacy) A protocol $\pi$ that realizes the OT functionality is private with respect to a malicious receiver if for any PPT adversary Rec* and PPT distinguisher $D$ there exists a negligible function $\mu(\cdot)$ such that for all $n$, except with probability $\mu(n)$ over $\operatorname{PExec}_{\operatorname{Rec}^{*}}\left(1^{n}\right)=\left(\left(m_{s}^{1}, m_{r}\right), \sigma, r_{\operatorname{Rec}}\right)$, there exists a bit $b$, such that for any strings $s_{0}, s_{1}$ and $s^{*}$ and auxiliary input $z$,

$$
\begin{aligned}
& \mid \operatorname{Pr}\left[m_{s}^{2} \leftarrow \operatorname{Sen}_{2}\left(\sigma, m_{r},\left(s_{0}, s_{1}\right)\right): D\left(1^{n}, z, r_{\operatorname{Rec}},\left(m_{s}^{1}, m_{s}^{2}\right)\right)=1\right] \\
& \quad-\mid \operatorname{Pr}\left[\left(x_{b} \leftarrow s_{b} ; x_{1-b} \leftarrow\{0,1\}^{\ell(n)} ; m_{s}^{2} \leftarrow \operatorname{Sen}_{2}\left(\sigma, m_{r},\left(x_{0}, x_{1}\right)\right):\right.\right. \\
& \quad D\left(1^{n}, z, r_{\operatorname{Rec}},\left(m_{s}^{1}, m_{s}^{2}\right)\right)=1 \mid \leq \mu(n)
\end{aligned}
$$

Next, let $\left\langle\operatorname{Sen} *\left(s_{0}, s_{1}\right), \operatorname{Rec}(b)\right\rangle\left(1^{n}\right)$ denote the random variable describing the corrupted sender's output when interacting with Rec that is invoked on inputs $b$.

Definition 2.13. (Receiver's privacy) A protocol $\pi$ that realizes the OT functionality is private with respect to a malicious sender if for any PPT adversary Sen* corrupting Sen the following distributions are indistinguishable

$$
\left.\left\{\operatorname{View}_{\text {Sen }}\left[\operatorname{Sen}^{*}\left(1^{n}\right), \operatorname{Rec}\left(1^{n}, 0\right)\right]\right)\right\} \stackrel{\mathrm{c}}{\approx}\left\{\operatorname{View}_{\text {Sen }}\left[\operatorname{Sen}^{*}\left(1^{n}\right), \operatorname{Rec}\left(1^{n}, 1\right)\right]\right\}
$$

\section{4-Round Coin-Tossing from Discrete Logarithm}

In this section, we present a 4-round coin-tossing protocol, computing the functionality $\left(1^{n}, 1^{n}\right) \mapsto\left(U_{t}, U_{t}\right)$ where $U_{t}$ is a random element in $\mathbb{Z}_{q}$, that is based on the hardness of the discrete logarithm problem. Namely, the parties use an extension of Pedersen's trapdoor commitment scheme [57] that is based on $n$ generators. Basically, party $P_{1}$ computes the generators for $P_{2}$ 's commitment scheme using pairs of shares and then reveals the discrete logarithm of half of the shares by responding to a random challenge given by $P_{2}$. Looking ahead, this allows to construct a simulator that extracts a trapdoor for this commitment scheme using rewinding which, in turn, allows the equivocation of the committed message. Forcing a particular outcome when $P_{2}$ is corrupted is carried out by first observing the decommitted value of $P_{2}$ and then rewinding, where in the second execution the simulator programs its input according to the outcome it received from the trusted party. 
We note that for both corruption cases, we construct universal simulators (namely, simulators that do not depend on the code of the adversary), that run in strict polynomialtime and induce $1 / p$-security. The simulator for a corrupted $P_{1}$ can be modified into an expected time simulator with full security as in the usual sense. The security of $P_{2}$ cannot be further enhanced as it learns the coin-tossing outcome after the third round and may choose to abort right after. Essentially, the problem is acute when the adversary's nonaborting probability in the last message is negligible, as it prevents from generating a view that is consistent with the coin-tossing outcome even using rewinding. Conditioned on this event, we prove that the difference between the simulated and real views is at most $1 / p(n)$.

We are now ready to present our protocol in details.

\section{Protocol 1. (Protocol $\left.\pi_{\mathrm{COIN}}\right)$}

Public Parameters The description of a group $\mathbb{G}$ of prime order $q$ and a generator $g$.

\section{The protocol}

1. $\mathbf{P}_{\mathbf{1}} \rightarrow \mathbf{P}_{\mathbf{2}}$ Pick random elements $t_{0}^{1}, t_{1}^{1}, \ldots, t_{0}^{n}, t_{1}^{n} \leftarrow \mathbb{Z}_{q}$ and sends $P_{2}$ the pairs $\left(h_{0}^{1}, h_{1}^{1}\right), \ldots,\left(h_{0}^{n}, h_{1}^{n}\right)$, where $h_{b}^{i}=g^{t_{0}^{i}}$ for all $b \in\{0,1\}$ and $i \in[n]$.

2. $\mathbf{P}_{2} \rightarrow \mathbf{P}_{1}$ Pick random elements $m, s_{1}, \ldots, s_{n} \leftarrow \mathbb{Z}_{q}$ and compute $\sigma=g^{m}\left(h_{0}^{1} h_{1}^{1}\right)^{s_{1}}$ $\cdots\left(h_{0}^{n} h_{1}^{n}\right)^{s_{n}}$. Select random bits $e_{1}, \ldots, e_{n}$ and send $\sigma, e_{1}, \ldots, e_{n}$ to $P_{1}$.

3. $\mathbf{P}_{\mathbf{1}} \rightarrow \mathbf{P}_{\mathbf{2}}$ Pick a random $m^{\prime} \leftarrow \mathbb{Z}_{q}$ and send $m^{\prime}, t_{e_{1}}^{1}, \ldots, t_{e_{n}}^{n}$ to $P_{2}$.

4. $\mathbf{P}_{\mathbf{2}} \rightarrow \mathbf{P}_{\mathbf{1}}$ Compute the coin-tossing outcome as $m+m^{\prime} \bmod p$ and send $m, s_{1}, \ldots, s_{n}$ to $P_{1}$.

Theorem 3.1. Assume that the discrete logarithm assumption holds in $\mathbb{G}$. Then Protocol 2 securely realizes $\mathcal{F}_{\text {CoIN }}$ in four rounds with $1 /$ p-security.

Proof. We consider each corruption case separately.

$P_{1}$ is corrupted On a high level, in order to simulate $P_{1}$ we construct a simulator $\mathcal{S}$ that extracts the trapdoor for one of the pairs $h_{0}^{i}, h_{1}^{i}$ sent in the first message, namely the discrete logarithm of both elements in the pair with respect to $g$, and then uses that to equivocate $P_{2}$ 's commitment in the last message. More precisely, for any probabilistic polynomial-time adversary $\mathcal{A}$ controlling $P_{1}$ we define a simulator $\mathcal{S}$ that is given an input $m_{o}$ from $\mathcal{F}_{\text {CoIN }}$ and proceeds as follows:

1. $\mathcal{S}$ internally invokes $\mathcal{A}$. Upon receiving the first message from $\mathcal{A}$, it feeds $\mathcal{A}$ with a second message generated using the honest $P_{2}$ 's strategy. Let $\sigma, e_{1}, \ldots, e_{n}$ be the message fed to $\mathcal{A}$ and $m, s_{1}, \ldots, s_{n}$ be the randomness used to generate the forth message (which is determined by the second message).

2. If $\mathcal{A}$ aborts before providing the third message, $\mathcal{S}$ halts outputting $\perp$. If $\mathcal{A}$ provides a third message, then $\mathcal{S}$ stalls the main execution and proceeds to rewind $\mathcal{A}$. Specifically, $\mathcal{S}$ rewinds $\mathcal{A}$ to the second message and supplies a different second message by sampling uniformly random coins for the honest $P_{2}$ 's strategy. Let $\tilde{e}_{1}, \ldots, \tilde{e}_{n}$ be the bits sent within the rewinded second message. If $\mathcal{A}$ responds, then $\mathcal{S}$ finds an index $j$ such that $\tilde{e}_{j} \neq e_{j}$. Note that such an index $j$ implies that $\mathcal{S}$ now has $t_{0}$ and $t_{1}$ such that $h_{0}^{j}=g^{t_{0}}$ and $h_{1}^{j}=g^{t_{1}}$. Else, if $\mathcal{A}$ aborts then $\mathcal{S}$ rewinds 
$\mathcal{A}$ to the second message and tries another freshly generated second message. $\mathcal{S}$ repeats this procedure $n p(n)$ times and outputs fail if (1) the challenges $\tilde{e}_{1}, \ldots, \tilde{e}_{n}$ are identical to $e_{1}, \ldots, e_{n}$ in any of the attempts or, (2) in case all the attempts were unsuccessful.

3. Finally, $\mathcal{S}$ proceeds to complete the main execution conditioned on not outputting fail. Let $m^{\prime}$ be part of the third message supplied by $\mathcal{A}$ and let $\sigma$ be the message fed to $\mathcal{A}$ as part of the second message. $\mathcal{S}$ computes

$$
\tilde{s}_{j}=\left(m-m_{o}+m^{\prime}+\left(t_{0}+t_{1}\right) s_{j}\right) /\left(t_{0}+t_{1}\right) \bmod p
$$

and for all other $i, \tilde{s}_{i}=s_{i}$. As the final message $\mathcal{S}$ feeds $\mathcal{A}$ with $\left(m_{o}-m^{\prime}\right), \tilde{s}_{1}, \ldots, \tilde{s}_{n}$.

We first argue for the correctness of the simulation. This follows from the ability to equivocate the commitment employed by $P_{2}$ once the discrete logarithm of one of the $h_{0}^{i} h_{1}^{i}$ elements is known to the simulator. More formally, let $j$ be as in the simulation for which the simulator obtains $t_{0}$ and $t_{1}$ such that $h_{0}^{j}=g^{t_{0}}$ and $h_{1}^{i}=g^{t_{1}}$. Moreover, let $\sigma=g^{m}\left(h_{0}^{1} h_{1}^{1}\right)^{s_{1}} \cdots\left(h_{0}^{n} h_{1}^{n}\right)^{s_{n}}$ as computed by the simulator in the second message of the simulation (note that $\sigma$ is fixed once for the entire simulation and is never modified). We focus our attention on the product $g^{m}\left(h_{0}^{j} h_{1}^{j}\right)^{s_{j}}$, where $s_{j}$ is the randomness revealed by the simulator in the third message. An important observation here is that it is sufficient to equivocate this product in order to equivocate the entire commitment. Namely, if the simulator can come up with two distinct pairs $\left(m, s_{j}\right)$ and $\left(\tilde{m}, \tilde{s}_{j}\right)$ such that $g^{m}\left(h_{0}^{j} h_{1}^{j}\right)^{s_{j}}=$ $g^{\tilde{m}}\left(h_{0}^{j} h_{1}^{j}\right)^{\tilde{s}_{j}}$, then it is possible to conclude two distinct openings with respect to the commitment used by $P_{2}$ by reusing the same $\left\{s_{i}\right\}_{i \neq j}$. Finally, since the simulator obtains $t_{0}$ and $t_{1}$ as above, it can conclude the discrete logarithm of $h_{0}^{j} h_{1}^{j}$ relative to $g$ which corresponds to $t_{0}+t_{1}$. Putting it all together, the simulator can easily equivocate $\ell=$ $g^{m}\left(h_{0}^{j} h_{1}^{j}\right)^{s_{j}}$ into the message $m_{o}-m^{\prime}$ (which will imply that the two shares yield $m_{o}$ ), by computing $\tilde{s}_{j}$ as follows. Consider the linear equation implied in the exponent of $\ell$ which equals $m+\left(t_{0}+t_{1}\right) s_{j}$, then $m+\left(t_{0}+t_{1}\right) s_{j}=m_{o}-m^{\prime}+\left(t_{0}+t_{1}\right) \tilde{s}_{j}$, which implies that $\tilde{s}_{j}=\left(m-m_{o}+m^{\prime}+\left(t_{0}+t_{1}\right) s_{j}\right) /\left(t_{0}+t_{1}\right) \bmod p$. Next we prove that. $\square$

Claim 3.1. There exists a negligible function negl( $\cdot)$ for which $\mathcal{S}$ outputs fail with probability at most $\frac{1}{p(n)}+\operatorname{negl}(n)$.

Proof. First, we consider a hybrid simulator $\tilde{\mathcal{S}}$ that instead of rewinding only $n p(n)$ times, repeatedly rewinds until it successfully obtains two responses from $\mathcal{A}$ relative to the third message. Moreover, $\tilde{\mathcal{S}}$ does not abort if the same challenge message occurs for a second time. We will next argue that the expected running time of $\tilde{\mathcal{S}}$ is polynomial. Let $\varepsilon$ denote the probability that $\mathcal{A}$ answers correctly on the third message. We consider two cases: (1) $\mathcal{A}$ aborts in the first simulated run (which occurs with probability $1-\varepsilon$ ). In this case, the simulator outputs $\perp$. (2) $\mathcal{A}$ does not abort in the first simulated run (which occurs with probability $\varepsilon$ ). In this case, the expected number of rewinding attempts $\tilde{\mathcal{S}}$ performs before $\mathcal{A}$ provides another valid third message is $\frac{1}{\varepsilon}$. Therefore, the expected number of times of $\tilde{\mathcal{S}}$ rewinds $\mathcal{A}$ is 


$$
(1-\varepsilon)+\varepsilon \frac{1}{\varepsilon}=O(1) .
$$

Next, we bound the probability of the strict simulator $\mathcal{S}$ outputting fail by computing the probability that it outputs fail in each of the cases. (1) The probability that $\mathcal{A}$ does not provide a third message within the $n p(n)$ attempts can be bounded using the Markov inequality, as the probability that $\tilde{\mathcal{S}}$ carries out more than $n p(n)$ rewinding attempts is at most $\frac{O(1)}{n p(n)}<\frac{1}{2 p(n)} ;(2)$ Next, the probability that $\mathcal{S}$ fails due to the event that the same challenge occurred twice can be bounded using a union bound argument which yields a value bounded by $n p(n) \times \frac{1}{2^{n}}$. We conclude that the overall probability that $\mathcal{S}$ outputs fail is bounded by $\frac{1}{2 p(n)}+\frac{n p(n)}{2^{n}}<\frac{1}{p(n)}$.

Claim 3.2. The following two distribution ensembles are computationally $\frac{1}{p(n)}$ indistinguishable,

$$
\begin{aligned}
& \left\{\operatorname{View}_{\pi_{\mathrm{COIN}}, \mathcal{A}(z)}(n)\right\}_{n \in \mathbb{N}, z \in\{0,1\}^{*}} \\
& \quad \stackrel{1 / \mathrm{p}}{\approx}\left\{\operatorname{View}_{\mathcal{F}_{\mathrm{COIN}}, \mathcal{S}(z)}(n)\right\}_{n \in \mathbb{N}, z \in\{0,1\}^{*}} .
\end{aligned}
$$

Proof. Finally, we wish to claim that the adversary's view in both real and simulated executions is identically distributed conditioned on the event that $\mathcal{S}$ does not output fail or abort. Note that the adversary's view is comprised from $\sigma, e_{1}, \ldots, e_{n}$ in the second message, and $m_{o}-m^{\prime}, s_{1}, \ldots, s_{j-1}, \tilde{s}_{j}, s_{j+1}, \ldots, s_{n}$ in the fourth message. Moreover, the second message is generated as in the real execution (and thus is distributed identically to the corresponding message in the real execution), whereas the fourth message is generated by first producing a real execution message and then equivocating the outcome commitment. We claim that the fourth simulated message is identically distributed to the fourth real message. On a high level, this is due to the fact that $m_{o}$ and $m^{\prime}$ are picked uniformly at random by $\mathcal{F}_{\mathrm{COIN}}$ and $\mathcal{S}$, respectively, and so $m_{o}-m^{\prime}$ is a uniformly distributed element in $\mathbb{Z}_{q}$. Moreover, $\tilde{s}_{j}$ depends on the distribution of $s_{j}$ which is uniformly random in $\mathbb{Z}_{q}$ as well.

More formally, our construction implies that the real and simulated views are indistinguishable relative to the partial views where the adversary aborts before sending the third message. It therefore suffices to show that the adversary's views are indistinguishable conditioned on not aborting in the simulation. More precisely, we prove that the distribution of $m_{o}-m^{\prime}, \tilde{s}_{1}, \ldots, \tilde{s}_{n}$ in the simulated view is identically distributed to the real view conditioned on $m_{o}$ being the outcome of the coin-tossing functionality, $m^{\prime}$ being the adversary's share, $\sigma$ being the second message and the adversary not aborting in the third message. It follows from our simulation that the distributions of $\tilde{s}_{i}$ for $i \neq j$ are identical as in both executions these values are sampled uniformly. Now, given that these values are already fixed, there exist unique values $m$ and $\tilde{s}_{j}$ that can be sent as part of the fourth message, which yield a consistent view with $m_{o}$. Hence, the views are identically distributed.

From Claim 3.1, we know that the probability $\mathcal{S}$ aborts is at most $\frac{1}{p(n)}+\operatorname{negl}(n)$. Therefore, 


$$
\operatorname{Pr}\left[\operatorname{View}_{\mathcal{F}_{\mathrm{COIN}}, \mathcal{S}(z)}(n) \neq \perp\right] \geq 1-\frac{1}{p(n)}-\operatorname{negl}(n) .
$$

Combining this claim with the fact that the simulated non-aborted view is identical to the real view, we obtain for every PPT distinguisher $D$ there exists a negligible function negl $(\cdot)$ such that for all sufficiently large $n$

$\left|\operatorname{Pr}\left[D\left(\operatorname{View}_{\mathcal{F}_{\mathrm{COIN}}, \mathcal{S}(z)}(n)\right)=1\right]-\operatorname{Pr}\left[D\left(\operatorname{View}_{\pi_{\mathrm{COIN}}, \mathcal{A}(z)}(n)\right)=1\right]\right|<\frac{1}{p(n)}+\frac{1}{\operatorname{negl}(n)}$.

$P_{2}$ is corrupted Informally, in case $P_{2}$ is corrupted the simulator extracts the committed message from $\mathcal{A}$ and then provides a share in the third message that is consistent with $m_{o}$ and $\mathcal{A}$ 's share. More precisely, for any probabilistic polynomial-time adversary $\mathcal{A}$ controlling $P_{2}$ we define a simulator $\mathcal{S}$ that is given an input $m_{o}$ from $\mathcal{F}_{\text {COIN }}$ and proceeds as follows:

1. $\mathcal{S}$ internally invokes $\mathcal{A}$ and computes the first message of the protocol as would have computed by the honest $P_{1}$. Namely, $\mathcal{S}$ picks random elements $t_{0}^{1}, t_{1}^{1}, \ldots, t_{0}^{n}, t_{1}^{n} \leftarrow$ $\mathbb{Z}_{q}$ and sends $\mathcal{A}$ the pairs $\left(h_{0}^{1}, h_{1}^{1}\right), \ldots,\left(h_{0}^{n}, h_{1}^{n}\right)$, where $h_{b}^{i}=g^{t_{0}^{i}}$ for every $b \in$ $\{0,1\}$ and $i \in[n]$. Let $\sigma, e_{1}, \ldots, e_{n}$ be the message replied by $\mathcal{A}$.

2. Next, $\mathcal{S}$ performs the following $n p(n)$ times:

- $\mathcal{S}$ picks a random $m^{\prime} \leftarrow \mathbb{Z}_{q}$ and sends $m^{\prime}, t_{e_{1}}^{1}, \ldots, t_{e_{n}}^{n}$ to $P_{2}$.

If at any iteration $\mathcal{A}$ provides a valid fourth message $m, s_{1}, \ldots, s_{n}$, then $\mathcal{S}$ rewinds $\mathcal{A}$ to the third message. Next, upon receiving $m_{o}$ from the ideal functionality, $\mathcal{S}$ supplies $\mathcal{A}$ with a third message $m_{o}-m, t_{e_{1}}^{1}, \ldots, t_{e_{n}}^{n}$ and completes the execution. If $\mathcal{A}$ aborts in all the $n p(n)$ attempts, $\mathcal{S}$ simply outputs the transcript from the first iteration.

We first prove that if the discrete logarithm assumption is hard in $\mathbb{G}$ then $\mathcal{A}$ cannot open $\sigma$ in two different valid ways as it violates this hardness assumption.

Claim 3.3. Assume that the discrete logarithm assumption holds in $\mathbb{G}$. Then, except with negligible probability, A cannot provide two tuples $m_{1}, s_{1}^{1}, \ldots, s_{n}^{1}$ and $m_{2}, s_{1}^{2}, \ldots, s_{n}^{2}$ for which $m_{1} \neq m_{2}$, that correspond to valid openings of $\sigma$.

Proof. Assume for contradiction that there exists an adversary $\mathcal{A}$ that can provide two valid distinct decommitments in the fourth round of the protocol with non-negligible probability. We show how to construct an adversary $\mathcal{B}$ that violates the discrete logarithm assumption relative to $\mathbb{G}$. On a high-level, upon given input $\left(g^{\prime}, h^{\prime}\right), \mathcal{B}$ sets $g=g^{\prime}$ and picks all $\left(h_{0}^{i}, h_{1}^{i}\right)$ pairs honestly with the exception that $h_{b}^{j}=h^{\prime}$ for a randomly chosen $b \in\{0,1\}$ and $j \in[n]$. Next, given two openings $m_{1}, s_{1}^{1}, \ldots, s_{n}^{1}$ and $m_{2}, s_{1}^{2}, \ldots, s_{n}^{2}, \mathcal{B}$ computes the discrete logarithm of $h^{\prime}$ with respect to $g=g^{\prime}$. More precisely, denote by $t_{b}^{i}$ the discrete logarithm of $h_{b}^{i}$ with respect to $g$ for all $b \in\{0,1\}$ and $i \in[n]$, i.e., $h_{b}^{i}=g^{t_{b}^{i}}$. Then it must hold that 


$$
m_{1}+\left(t_{0}^{1}+t_{1}^{1}\right) s_{1}^{1}+\cdots+\left(t_{0}^{n}+t_{1}^{n}\right) s_{n}^{1}=m_{2}+\left(t_{0}^{1}+t_{1}^{1}\right) s_{1}^{2}+\cdots+\left(t_{0}^{n}+t_{1}^{n}\right) s_{n}^{2}
$$

as $\mathcal{A}$ provides two openings to the same commitment $\sigma$. Therefore, it is simple to compute

$$
t_{b}^{j}=\left[m_{1}-m_{2}+\sum_{i \neq j}\left(t_{0}^{i}+t_{1}^{i}\right)\left(s_{i}^{1}-s_{i}^{2}\right)+t_{1-b}^{j}\left(s_{j}^{1}-s_{j}^{2}\right)\right] /\left(s_{j}^{2}-s_{j}^{1}\right)
$$

which implies that $\mathcal{B}$ violates the discrete logarithm assumption relative to $\mathbb{G}$.

Claim 3.4. The following two distribution ensembles are computationally $\frac{1}{p}$ indistinguishable,

$$
\begin{aligned}
& \left\{\operatorname{View}_{\pi_{\mathrm{COIN}}, \mathcal{A}(z)}(n)\right\}_{n \in \mathbb{N}, z \in\{0,1\}^{*}} \\
& \quad \stackrel{1 / p}{\approx}\left\{\operatorname{View}_{\mathcal{F}_{\mathrm{COIN}}, \mathcal{S}(z)}(n)\right\}_{n \in \mathbb{N}, z \in\{0,1\}^{*}} .
\end{aligned}
$$

Proof. Let $\delta$ be the probability of which $\mathcal{A}$ sends the fourth message. We consider two cases:

Case $\delta>\frac{1}{p(n)}$ In this case, the probability that $\mathcal{S}$ fails to extract $m$ within the $n p(n)$ trials is negligible in $n$. Moreover, it is easy to argue that whenever $\mathcal{S}$ extracts $m$, then the distribution generated by $\mathcal{S}$ is identically distributed to the real view conditioned on the adversary not equivocating. Specifically, as this event only occurs with negligible probability (as shown in Claim 3.3), the real and ideal views are statistically close.

Case $\delta<\frac{1}{p(n)}$ In this case, let $\epsilon$ be the probability that $\mathcal{S}$ fails to extract $m$ within the $n p(n)$ trials. Let $D_{a}$ be the distribution of the real view of the adversary conditioned on it aborting in the fourth step, and let $D_{b}$ be the real view conditioned on the adversary not aborting. Then we can express the distribution of $\mathcal{A}$ 's real view as a mixture of distributions as follows: ${ }^{4}$

$$
(1-\delta) D_{a}+\delta D_{b}
$$

The simulator on the other hand will generate a distribution as follows:

$$
(1-\epsilon) D_{a}+\epsilon\left((1-\delta) D_{a}+\delta D_{b}\right)
$$

Then the statistical distance between the two distributions can be computed as the difference

$$
\left\|(\delta-\epsilon \delta) D_{a}+(\epsilon \delta-\delta) D_{b}\right\|_{1}=\delta(1-\epsilon)\left\|\left(D_{a}-D_{b}\right)\right\|_{1}
$$

\footnotetext{
${ }^{4}$ More precisely, the real view can be obtained by first selecting $D_{a}$ with probability $\delta$ and $D_{b}$ otherwise, and then the selecting a random view in the particular distribution.
} 
which is bounded from above by $\delta<\frac{1}{p(n)}$. Hence the real and simulated view are $\frac{1}{p}$-indistinguishable.

\subsection{An Abstraction Using Homomorphic Trapdoor Commitment Schemes}

We further demonstrate how to abstract the protocol from Sect. 3 based on an homomorphic two-round trapdoor commitment scheme (cf. Sect. 2.3.1), denoted by $\pi_{\mathrm{COM}}=$ $\left(\pi_{\mathrm{Sen}}, \pi_{\mathrm{Rec}}\right)$.

\section{Protocol 2. (Protocol $\pi_{\mathrm{COIN}}$ )}

\section{The protocol:}

1. $P_{1}$ (playing the role of the receiver) generates $2 n$ pairs of instances of the first message in $\pi_{\mathrm{COM}}$ denoted by $\left(\left(\pi_{\operatorname{Rec}_{1}}^{0}, \pi_{\operatorname{Rec}_{1}}^{1}\right), \ldots,\left(\pi_{\operatorname{Rec}_{n}}^{0}, \pi_{\operatorname{Rec}_{n}}^{1}\right)\right)$ (with independent fresh randomness), and sends these pairs to $P_{2}$.

2. For all $j \in[n], P_{2}$ first combines each pair $\left(\pi_{\operatorname{Rec}_{j}}^{0}, \pi_{\operatorname{Rec}_{j}}^{1}\right)$ into a single instance

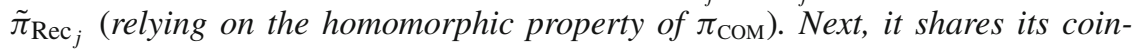
tossing share $m_{2}$ into $n$ shares $m_{2}^{1}, \ldots, m_{2}^{n}$ and commits to these shares by computing the response to $\tilde{\pi}_{\operatorname{Rec}_{j}}$, denote these responses by $\left(\pi_{\mathrm{Sen}_{1}}, \ldots, \pi_{\mathrm{Sen}_{n}}\right) . P_{2}$ additionally sends a random challenge $e \leftarrow\{0,1\}^{n}$.

3. Let $e=\left(e_{1}, \ldots, e_{n}\right)$. Then $P_{1}$ reveals the randomness it used for computing $\pi_{\operatorname{Rec}_{j}}^{e_{j}}$ for all $j \in[n]$, and further sends its coin-tossing share $m_{1}$.

4. $P_{2}$ verifies that $P_{1}$ generated the first message correctly with respect to challenge $e$. If all the verifications are accepting $P_{2}$ opens its commitments from Step 2 and $P_{1}$ verifies the validity of this opening. If all the verifications are accepting the parties output $m_{1}+m_{2}$ (where addition is computed in the corresponding group). Otherwise, $P_{1}$ aborts.

Intuitively speaking, Protocol 2 is proven similarly to the proof of Protocol 1. Namely, when $P_{1}$ is corrupted the simulator extracts one of the trapdoor pairs of the commitment scheme that enables to equivocate the corresponding receiver's share. On the other hand, when $P_{2}$ is corrupted, then the simulator behaves identically to the simulator of $P_{2}$ for Protocol 1. That is, the simulator extracts the committed message from the adversary and then rewinds it, providing a new third message that is consistent with $m_{o}$. It is simple to verify that the proof follows as for Protocol 1, described above in Sect. 3. Two additional constructions with security under the RSA and the factoring hardness assumptions are captured by our abstraction as well; see Sect. 2.3.1 for more details.

\section{Warmup: 4-Round 2PC Against Non-aborting Adversaries}

In this section, as a warmup, we present a 4-round two-party protocol for arbitrary functionalities, where both parties receive the output, in the presence of arbitrary malicious adversaries that are restricted to be non-aborting. We first introduce a 4-round oblivious- 
transfer protocol that securely computes functionality $\mathcal{F}_{\text {OТ }}:\left(\left(s_{0}, s_{1}\right), b\right) \mapsto\left(-, s_{b}\right)$ in the presence of non-aborting senders and receivers, where the receiver receives the output in the third round. Next, we obtain a 4-round two-party protocol with the same security guarantees by combining our oblivious-transfer protocol with [43]. In the following section, we rely on this protocol as a building block to construct another OT protocol that achieves $1 / p$-security against malicious (aborting) senders and full simulation-based security against non-aborting receivers.

First, we begin with a brief discussion on non-aborting adversaries. To model such adversaries, we will require parties to output a special reject message to indicate rejecting a conversation. Formally, a PPT adversary $\mathcal{A}$ controlling party $P_{1}$ (resp., $P_{2}$ ) in an interaction using protocol $\pi=\left\langle P_{1}, P_{2}\right\rangle$ with an honest party $P_{2}$ (resp. $P_{1}$ ), is said to be non-aborting in an execution of the protocol if at the end of the protocol $P_{2}$ (resp. $P_{1}$ ) does not reject the conversation. An adversary is said to be non-aborting if the probability with which the other party outputs reject is negligible.

We proceed with our oblivious-transfer protocol followed by our general two-party computation in Section 4.3.

\subsection{Building Blocks}

Our protocol relies on the following cryptographic building blocks:

Proof of Validity The receiver in our protocol uses a standard $\Sigma$-protocol WI-PoK for proving the knowledge of the discrete logarithm of one of the public keys it forwards the sender. The protocol ensures that there is at least one public key for which the receiver knows the discrete logarithm relative to some generator (where this corresponds to the public key for which the receiver does not know the secret key). Concretely, we consider a $\Sigma$-protocol $\pi_{\mathrm{DL}}^{\mathrm{WI}}$ for the following language [16],

$$
\mathcal{L}_{\mathrm{DL}}=\left\{(g, h, \mathbb{G}, q) \mid \exists u \in \mathbb{Z}_{q} \text { such that } h=g^{u}\right\} .
$$

We note that this proof is given for compound statements. Namely, the parties hold two statements for which the prover only knows one of the witnesses, but not both. It is a common technique by now to combine two $\Sigma$-protocols (even distinct ones) in a way that ensures that the prover knows at least one of the witnesses [15]. We note that the compound proof implies a perfect WI-PoK (namely, the view that is produced with respect to one witness is identical to a view that is produced with respect to the other witness). Consequently, even an unbounded verifier cannot tell which witness is used by the prover for proving the compound statement.

The El Gamal PKE [27] (see Appendix A.1.1) Intuitively speaking, the receiver chooses group elements that will be later viewed by the sender as El Gamal public keys. The key point is that the receiver must pick these elements in two distinct ways, which will be verified by the sender using the WI-PoK $\pi_{\mathrm{DL}}^{\mathrm{WI}}$. If indeed the receiver completes this proof correctly, then we can prove that there exists a public key for which the receiver does not know the trapdoor secret key. This will allow us to claim the privacy of one of the sender's inputs. On the other hand, if the receiver cheats then it may learn both of the sender's inputs. Nevertheless, in this case it will always be caught. 


\subsection{4-Round OT Against Non-aborting Adversaries}

In this section, we construct a 4-round OT protocol that guarantees full security assuming non-aborting adversaries. We note that an aborting receiver may violate the privacy of the sender and learn its both inputs while an aborting sender cannot violate the privacy of the receiver.

\section{Protocol 3. (Protocol $\pi_{\mathrm{OT}}$ )}

Public Parameters The description of a group $\mathbb{G}$ of prime order $q$.

Inputs The sender Sen holds $s_{0}, s_{1}$ and the receiver Rec holds a bit $b$.

\section{The protocol:}

\section{Sen $\rightarrow$ Rec}

(a) Sen picks a random generator $g \leftarrow \mathbb{G}$ and computes $h_{0}=g^{r_{0}}$ and $h_{1}=g^{r_{1}}$ where $r_{0}, r_{1} \leftarrow \mathbb{Z}_{q}$.

(b) Sen sends $g, h_{0}, h_{1}$ to Rec.

\section{2. $\operatorname{Rec} \rightarrow$ Sen}

(a) Rec generates two public keys according to the El Gamal PKE as follows: $\mathrm{PK}_{b}=g^{m}$ and $\mathrm{PK}_{1-b}=\left(h_{0} h_{1}\right)^{\tilde{m}}$ where $m, \tilde{m} \leftarrow \mathbb{Z}_{q}$. Rec sets $\mathrm{SK}=m$.

(b) Rec sends $\mathrm{PK}_{0}, \mathrm{PK}_{1}$ to Sen.

(c) Rec sends the first message of the WI-PoK for proving the knowledge of the discrete logarithms of either $\mathrm{PK}_{0}$ or $\mathrm{PK}_{1}$ with respect to $\left(h_{0} h_{1}\right)$ (namely, $\mathrm{Rec}$ sends the first message with respect to $\pi_{\mathrm{DL}}^{\mathrm{WI}}$ for the compound statement with $\mathrm{PK}_{0}$ and $\mathrm{PK}_{1}$ being the statements).

(d) Rec sends a challenge bit $\beta$.

\section{Sen $\rightarrow$ Rec}

(a) Sen computes ciphertexts $c_{0}, c_{1}$ as follows: $c_{0}=\left(g^{u_{0}}, \mathrm{PK}_{0}^{u_{0}} \cdot s_{0}\right)$ and $c_{1}=$ $\left(g^{u_{1}}, \mathrm{PK}_{1}^{u_{1}} \cdot s_{1}\right)$ where $u_{0}, u_{1} \leftarrow \mathbb{Z}_{q}$.

(b) Sen sends $c_{0}, c_{1}$ to Rec

(c) Sen sends the second message $e_{\mathrm{Sen}}$ for the WI-PoK protocol $\pi_{\mathrm{DL}}^{\mathrm{WI}}$ given by the receiver (recall that this message is a random challenge).

(d) Sen sends $r_{\beta}=\log _{g}\left(h_{\beta}\right)$

\section{4. $\operatorname{Rec} \rightarrow$ Sen}

(a) Upon receiving the sender's message, the receiver first checks if $r_{\beta}=\log _{g}\left(h_{\beta}\right)$. If this is not the case, it outputs reject and halts. Otherwise, it takes the ciphertexts $c_{0}=\left\langle c_{0}[1], c_{0}[2]\right\rangle$ and $c_{1}=\left\langle c_{1}[1], c_{1}[2]\right\rangle$ and computes $s_{b}$ by decrypting $c_{b}$ under $\mathrm{SK}_{b}$. More precisely, it computes $s_{b}=c_{b}[2] /\left(c_{b}[1]\right)^{\mathrm{SK}}$.

(b) Rec sends the last message for the WI-PoK protocol $\pi_{\mathrm{DL}}^{\mathrm{WI}}$.

Finally, if the proof using $\pi_{\mathrm{DL}}^{\mathrm{WI}}$ is not convincing (or if any of the messages from Rec were ill-formed), Sen outputs reject. Similarly if any of the messages from Rec were ill-formed then Rec outputs reject.

Theorem 4.1. (Warmup) Assume that the Decisional Diffie-Hellman assumption holds in $\mathbb{G}$ and that $\pi_{\mathrm{DL}}^{\mathrm{WI}}$ is a perfectly 3-round WI-PoK protocol. Then, Protocol 3 is a 4-round 
protocol, where the receiver receives the output in the third round, that securely realizes $\mathcal{F}_{\mathrm{OT}}$ in the presence of non-aborting senders and non-aborting receivers. Furthermore, the protocol is receiver private according to Definition 2.13 (i.e., private against an (aborting) malicious sender).

Proof Overview First, in case the sender is corrupted the simulator plays the role of the honest receiver with input $b=0$ and extracts both $r_{0}$ and $r_{1}$. Next, the simulator uses these values in order to equivocate the public keys it sends to the adversary in the second message. Namely, upon extracting the discrete logarithms of both $h_{0}$ and $h_{1}$ the simulator knows the secret keys for both public keys and can decrypt both ciphertexts.

On the other hand, in case the receiver is corrupted, security is proven via a reduction to the IND-CPA security game of the El Gamal PKE. Namely, the simulator first extracts the receiver's secret exponent $\tilde{m}$ and the bit $b$ (from the WI-PoK $\pi_{\mathrm{DL}}^{\mathrm{WI}}$ ) and uses that information to complete the IND-CPA reduction by plugging in an external public key instead of $\left(h_{0} h_{1}\right)^{\widetilde{m}}$ and a ciphertext that either encrypts $s_{1-b}$ or a random independent message.

Correctness On a high-level, correctness follows from the correctness of the El Gamal PKE. Namely, given that the receiver knows the secret key $m$ for $\mathrm{PK}_{b}$, it can decrypt ciphertext $c_{b}$.

Proof. We consider each corruption case separately.

Sen is corrupted Recall that when the sender is corrupted we need to prove that it cannot learn anything about the bit $b$ while extracting both $s_{0}$ and $s_{1}$. More precisely, consider a non-aborting probabilistic polynomial-time adversary $\mathcal{A}$ controlling Sen. We define a simulator $\mathcal{S}$ that proceeds as follows:

1. $\mathcal{S}$ invokes $\mathcal{A}$ on its input and randomness of appropriate length.

2. Upon receiving from $\mathcal{A}$ the first message, $\mathcal{S}$ computes the second message honestly with input $b=0$.

3. Upon receiving $\mathcal{A}$ 's third message, $\mathcal{S}$ records $r_{\beta}$. Next, it stalls the main execution and proceeds to rewind $\mathcal{A}$. Specifically, $\mathcal{S}$ rewinds $\mathcal{A}$ to the second message and supplies a bit $1-\beta$. Upon receiving $r_{1-\beta}, \mathcal{S}$ completes the main execution honestly using $b=0$ and decrypts both ciphertexts as follows. $\mathcal{S}$ uses $\mathrm{SK}_{0}=\mathrm{SK}$ to decrypt $c_{0}$ as the honest receiver would do. Moreover, $\mathcal{S}$ fixes $\mathrm{SK}_{1}=\left(r_{0}+r_{1}\right) \tilde{m}$ and uses $\mathrm{SK}_{1}$ to decrypt $c_{1}$.

4. Finally, $\mathcal{S}$ forwards $\left(s_{0}, s_{1}\right)$ to $\mathcal{F}_{\text {Oт }}$ and halts, outputting whatever $\mathcal{A}$ does.

Clearly, $\mathcal{S}$ runs in strict polynomial-time. We first prove the correctness of simulation. First, we know that except with negligible probability the simulator obtains the correct discrete logarithm of $h_{\beta}$. This is because the probability with which a non-aborting adversary fails to give the correct discrete logarithm is negligible. Therefore, it suffices to show that the simulator correctly extracts $s_{1}$. Recall that for $b=1$ the honest receiver computes $s_{1}=c_{1}[2] /\left(c_{1}[1]\right)^{\mathrm{SK}_{1}}$. Then we claim that this is equivalent to the computation carried out by the simulator, as $\mathrm{SK}_{1}$ amounts in this case to the discrete logarithm of $\mathrm{PK}_{1}$ relative to generator $g$. Next, we prove that, 
Claim 4.1. The following two distribution ensembles are identical,

$$
\begin{aligned}
& \left\{\operatorname{View}_{\pi_{\mathrm{OT}}, \mathcal{A}(z)}\left(n,\left(s_{0}, s_{1}\right), b\right)\right\}_{n \in \mathbb{N}, s_{0}, s_{1}, b, z \in\{0,1\}^{*}} \\
& \quad \equiv\left\{\operatorname{View}_{\mathcal{F}_{\mathrm{OT}}, \mathcal{S}(z)}\left(n,\left(s_{0}, s_{1}\right), b\right)\right\}_{n \in \mathbb{N}, s_{0}, s_{1}, b, z \in\{0,1\}^{*}} .
\end{aligned}
$$

Proof. The proof follows due to the fact that the receiver's bit $b$ is information theoretically hidden given $\mathrm{PK}_{0}, \mathrm{PK}_{1}$ and the WI-PoK transcript of $\pi_{\mathrm{DL}}^{\mathrm{WI}}$. More concretely, given any pair $\left(\mathrm{PK}_{0}, \mathrm{PK}_{1}\right)$ there always exist $m^{0}, \tilde{m}^{0}$ and $m^{1}, \tilde{m}^{1}$ for which $\mathrm{PK}_{0}=$ $g^{m_{0}}=\left(h_{0} h_{1}\right)^{\widetilde{m}_{0}}$ and $\mathrm{PK}_{1}=g^{m_{1}}=\left(h_{0} h_{1}\right)^{\widetilde{m}_{1}}$. Moreover, the WI-PoK $\pi_{\mathrm{DL}}^{\mathrm{WI}}$ is a perfect witness-indistinguishable proof, which implies that even an unbounded verifier cannot extract $b$ (as discussed above, this is the case even for the compound proof, since the receiver proves that it knows a discrete logarithm relative to either $\mathrm{PK}_{0}$ or $\mathrm{PK}_{1}$ ).

It therefore holds that even if the sender aborts prematurely, it cannot obtain any information about $b$. This also proves that the protocol is receiver private.

Rec is corrupted In this case, we need to prove that the corrupted receiver cannot learn anything about the sender's other input $s_{1-b}$ while extracting $b$. More precisely, for any non-aborting probabilistic polynomial-time adversary $\mathcal{A}$ controlling $\operatorname{Rec}$ we define a simulator $\mathcal{S}$ that proceeds as follows:

1. $\mathcal{S}$ invokes $\mathcal{A}$ on its input and randomness of the appropriate length.

2. $\mathcal{S}$ plays the role of the honest sender with arbitrary inputs $\left(s_{0}^{\prime}, s_{1}^{\prime}\right)$. Upon completing the execution successfully, $\mathcal{S}$ stalls the main execution and proceeds to rewind $\mathcal{A}$. Specifically, $\mathcal{S}$ rewinds $\mathcal{A}$ to the third message and supplies a different second message for $\pi_{\mathrm{DL}}^{\mathrm{ZK}}$ by sampling uniformly random new challenge $e_{\mathrm{Sen}}^{\prime}$. If $e_{\mathrm{Sen}}=e_{\mathrm{Sen}}^{\prime}$, i.e., the challenge is identical, then $\mathcal{S}$ aborts. Otherwise, it feeds the challenge to $\mathcal{A}$ as part of the second message. Finally, $\mathcal{S}$ runs the extractor for the WI-PoK $\pi_{\mathrm{DL}}^{\mathrm{WI}}$ and extracts the bit $b$ and the discrete logarithm of $\mathrm{PK}_{1-b}$.

Specifically, let $\gamma$ be such that the simulator extracts $\tilde{m}$ with respect to $\mathrm{PK}_{\gamma}$. Then $\mathcal{S}$ fixes the bit $b=1-\gamma$.

3. $\mathcal{S}$ submits $b$ to $\mathcal{F}_{\text {OT }}$, and receives $s_{b}$.

4. $\mathcal{S}$ rewinds $\mathcal{A}$ to the third message and computes it based on $s_{b}$ and random $s_{1-b}$.

5. $\mathcal{S}$ halts, outputting whatever $\mathcal{A}$ does.

Note first that the simulator runs in polynomial-time and that the probability it aborts is negligible. Moreover, we prove that the simulated and real views are computationally indistinguishable via a reduction to the security of the El Gamal PKE. Namely, we prove the following claim,

Claim 4.2. The following two distribution ensembles are computationally indistinguishable,

$$
\begin{aligned}
& \left\{\text { View }_{\pi_{\mathrm{OT}}, \mathcal{A}(z)}\left(n,\left(s_{0}, s_{1}\right), b\right)\right\}_{n \in \mathbb{N}, s_{0}, s_{1}, b, z \in\{0,1\}^{*}} \\
& \quad \stackrel{\mathrm{c}}{\approx}\left\{\operatorname{View}_{\mathcal{F}_{\mathrm{OT}}, \mathcal{S}(z)}\left(n,\left(s_{0}, s_{1}\right), b\right)\right\}_{n \in \mathbb{N}, s_{0}, s_{1}, b, z \in\{0,1\}^{*}} .
\end{aligned}
$$


Proof. Assume by contradiction that these two views are distinguishable by a PPT distinguisher $D$. We construct an adversary $\mathcal{A}^{\prime}$ that breaks the security of the El Gamal PKE as follows. Recall that $\mathcal{A}^{\prime}$ externally communicates with a challenger that provides to it a public key $\mathrm{PK}=\langle g, h\rangle$. Upon receiving $\mathrm{PK}$ and $\left(s_{0}, s_{1}\right)$ as the auxiliary input, $\mathcal{A}^{\prime}$ picks a random bit $\beta^{\prime}$ and sets $h_{\beta^{\prime}}=g^{x}$ for some random $x \leftarrow \mathbb{Z}_{q}$. In addition, $\mathcal{A}^{\prime}$ sets $h_{1-\beta^{\prime}}=h / h_{\beta^{\prime}}$. $\mathcal{A}^{\prime}$ invokes $\mathcal{A}$ internally and forwards it the first message of the protocol $g, h_{0}, h_{1}$. Upon receiving $\mathcal{A}$ 's second message, $\mathcal{A}^{\prime}$ aborts if $\beta^{\prime} \neq \beta$. Else, $\mathcal{A}^{\prime}$ completes the execution using arbitrary $\left(s_{0}, s_{1}\right)$. Upon completing the execution successfully, $\mathcal{A}^{\prime}$ extracts $b$ and the discrete logarithm of $\mathrm{PK}_{1-b}$ exactly as done in the simulation. This is possible except with negligible probability because the receiver is non-aborting. Finally, $\mathcal{A}^{\prime}$ submits to its challenger the two messages $s_{1-b}^{\tilde{m}^{-1}}$ and $t$ for $t \leftarrow \mathbb{Z}_{q}$, receiving back a challenge ciphertext $c=\left\langle c_{0}^{\prime}, c_{1}^{\prime}\right\rangle$ that encrypts one of these plaintexts at random. $\mathcal{A}^{\prime}$ computes $\left\langle\left(c_{0}^{\prime}\right)^{\widetilde{m}},\left(c_{1}^{\prime}\right)^{\tilde{m}}\right\rangle$ (and rerandomizes the ciphertext by multiplying the outcome with a random encryption of zero), and plugs the outcome as the ciphertext that encrypts $s_{1-b}$ and halts. Finally, $\mathcal{A}^{\prime}$ invokes $D$ on the joint distribution of $\left(s_{0}, s_{1}\right)$ and the adversary's output and outputs whatever $D$ does.

We now consider two cases:

1. In the first case the challenge $c^{\prime}$ is an encryption of $s_{1-b}^{\tilde{m}^{-1}}$. We claim that in this case the adversary's view is distributed as in the real execution. This is because the challenge ciphertext $\left\langle\left(c_{0}^{\prime}\right)^{\tilde{m}},\left(c_{1}^{\prime}\right)^{\widetilde{m}}\right\rangle$ corresponds to a random ciphertext that encrypts the plaintext $s_{1-b}$ relative to $\mathrm{PK}_{1-b}$.

2. On the other hand, in case the challenge $c^{\prime}$ is an encryption of a random element $t$, then the adversary's view is distributed as in the simulation, as the simulator does not know $s_{1-b}$ and hence uses a random input instead of the real value.

In both cases, the first message of the reduction is identically distributed to the first message in the corresponding execution. Moreover, the distribution of the first message for $\beta^{\prime}=0$ is identical to the distribution for the case that $\beta^{\prime}=1$.

More formally, assume that

$$
\begin{aligned}
& \mid \operatorname{Pr}\left[D\left(\mathbf{V i e w}_{\pi_{\mathrm{OT}}, \mathcal{A}(z)}\left(n,\left(s_{0}, s_{1}\right), b\right)\right)=1\right] \\
& \quad-\operatorname{Pr}\left[D\left(\operatorname{View}_{\mathcal{F}_{\mathrm{OT}}, \mathcal{S}(z)}\left(n,\left(s_{0}, s_{1}\right), b\right)\right)=1\right] \mid \geq \varepsilon(n) .
\end{aligned}
$$

Then, it holds that

$$
\operatorname{Pr}\left[\operatorname{ADV}_{\Pi, \mathcal{A}^{\prime}}(n)=1\right] \geq \frac{1}{2}+\frac{\varepsilon(n)}{2}
$$

condition on the event for which $\beta^{\prime}=\beta$. This is proven as follows,

$$
\begin{aligned}
\operatorname{Pr} & \left.\operatorname{ADV}_{\Pi, \mathcal{A}^{\prime}}(n)=1\right] \\
& =\frac{1}{2}\left(\operatorname{Pr}\left[\operatorname{ADV}_{\Pi, \mathcal{A}^{\prime}}(n)=1 \mid b=0\right]+\operatorname{Pr}\left[\operatorname{ADV}_{\Pi, \mathcal{A}^{\prime}}(n)=1 \mid b=1\right]\right) \\
& =\frac{1}{2}\left(\operatorname{Pr}\left[D\left(\operatorname{View}_{\pi_{\mathrm{OT}}, \mathcal{A}(z)}\left(n,\left(s_{0}, s_{1}\right), b\right)\right)=0\right]\right.
\end{aligned}
$$




$$
\begin{aligned}
& \left.+\operatorname{Pr}\left[D\left(\operatorname{View}_{\mathcal{F}_{\mathrm{OT}}, \mathcal{S}(z)}\left(n,\left(s_{0}, s_{1}\right), b\right)\right)=1\right]\right) \\
= & \frac{1}{2}\left(1-\operatorname{Pr}\left[D\left(\mathbf{V i e w}_{\pi_{\mathrm{OT}}, \mathcal{A}(z)}\left(n,\left(s_{0}, s_{1}\right), b\right)\right)=1\right]\right. \\
& \left.+\operatorname{Pr}\left[D\left(\operatorname{View}_{\mathcal{F}_{\mathrm{OT}}, \mathcal{S}(z)}\left(n,\left(s_{0}, s_{1}\right), b\right)\right)=1\right]\right) \\
= & \frac{1}{2}+\frac{1}{2} \mid \operatorname{Pr}\left[D\left(\operatorname{View}_{\mathcal{F}_{\mathrm{OT}}, \mathcal{S}(z)}\left(n,\left(s_{0}, s_{1}\right), b\right)\right)=1\right] \\
& -\operatorname{Pr}\left[D\left(\operatorname{View}_{\pi_{\mathrm{OT}}, \mathcal{A}(z)}\left(n,\left(s_{0}, s_{1}\right), b\right)\right)=1\right] \mid \\
\geq & \frac{1}{2}+\frac{\varepsilon(n)}{2} .
\end{aligned}
$$

\subsection{4-Round 2PC Against Non-aborting Adversaries}

First, we observe that we can repeat our OT protocol in parallel while guaranteeing the same security. This is because in case the sender is corrupted, information theoretic privacy is still maintained under parallel execution (as each OT execution is carried out with respect to an independent pair of keys). Moreover, the basic simulator can be extended for a simulator for the parallel execution, as input extraction follows easily in the presence of non-aborting adversaries. Using a standard hybrid argument, simulationbased security is further maintained in case the receiver is corrupted.

Next, obtaining general secure two-party computation is carried out by embedding the 2-round protocol of [43] within our second/third messages of our OT protocol. We briefly recall the high-level structure of the protocol in [43]. The authors provide a noninteractive protocol for securely computing any functionality between a sender and a receiver in the OT-hybrid, where both parties have inputs and only the receiver receives the output. Moreover, the sender is the sender in all OT invocations. More precisely, in a single message, the sender sends one message to the receiver and provides inputs to all OT instances in parallel. Whereas the receiver bases the queries to the OT functionality on its input, playing the receiver in all parallel instances. Next, based on the one-message received from the sender and the outputs from all OT instances, the receiver computes the result of the computation. Formally, we can capture the result in [43] in the following theorem statement.

Theorem 4.2. (Implicit in [43]) Assuming one-way functions, for every (one output) two-party functionality $f:\{0,1\}^{*} \times\{0,1\}^{*} \rightarrow\{0,1\}^{*}$ between a sender and a receiver, there is a protocol that securely realizes $f$ in the parallel OT-hybrid with the following features:

- The protocol involves a single message from the sender to the receiver.

- The receiver receives the output.

- The protocol makes black-box use of the underlying one-way function. 
Description of our Protocol We obtain a secure protocol by instantiating [43] with our four-message protocol from the previous section. All OT instances will be executed in parallel and the one-message sent by the sender is sent along with the third message of the OT protocol. Since the receiver obtains the result of the OT after receiving the third message of the protocol, along with the one-message sent as part of the [43] protocol, the receiver can compute the result after the third message.

We remark that we obtain a secure two-party protocol with the same security guarantees, namely security against malicious non-aborting senders and malicious non-aborting receivers. In the description above, only one party, namely the receiver, receives the output. However, if we want both parties to receive the output, the receiver can simply provide the output along with the fourth message to the sender. ${ }^{5}$

Formally, we obtain the following theorem.

Theorem 4.3. Assuming the Decisional Diffie-Hellman problem is hard, there exists a 4-round two-party protocol for any functionality, where both parties receive the output, that is fully secure in the presence non-aborting senders and non-aborting receivers.

Proof Sketch We consider each corruption case separately. When the receiver is corrupted, we will rely on the simulation provided by Theorem 4.2. Namely, the authors of [43] provide a simulator that upon receiving the inputs of the receiver to the parallel OT protocol obtains the output of the computation under $f$ from the ideal functionality and simulates the message sent from the sender to the receiver. Since we have a non-aborting receiver that provides a WI-PoK, following the receiver's simulation strategy of the previous section, we can extract the inputs of the receiver for the parallel OT invocations. In more detail, we consider a simulator that honestly generates the sender's messages with arbitrary inputs for the functionality being computed and then extracts the receiver's inputs to the OT by rewinding the WI-PoK. Then, we follow the simulation strategy of [43] to complete the simulation.

In order to achieve simulation when the sender is corrupted, we observe that, upon extracting the trapdoors, it is possible to set up the OT part in the second message from the receiver in such a way that the sender's inputs to the OT can be extracted with perfect simulation. Then relying on the simulation of the [43] simulator in the $\mathcal{F}_{\text {OT-hybrid we }}$ can obtain the real input of the sender which is forwarded to the ideal functionality. Internally, to complete the execution, the simulator uses a random input as the witness in the WI-PoK. Indistinguishability follows directly from the WI property of the WI-PoK and the sender privacy within the OT protocol.

\footnotetext{
${ }^{5}$ In order to ensure correctness, we can make sure that the computed function additionally outputs a MAC so that the sender is assured of the output the receiver sends. Alternatively, the output can also be encrypted (in case of an asymmetric function) under the sender's key, which the receiver can relay back to the sender.
} 


\section{4-Round 2PC with 1/p Sender Security and Full Security Against Non-aborting Receivers}

In this section, we extend our OT protocol from Sect. 4 and demonstrate how to achieve $1 / p$-simulation with respect to corrupted aborting senders while retaining the same guarantees against non-aborting receivers. Next, in Sect. 5.2, we show how to induce a general 2PC protocol with the same security guarantees. Our OT protocol is inspired by the recent result of Ostrovsky, Richelson and Scafuro [55]. Roughly speaking, the protocol in [55] provide a cut-and-choose mechanism to transform an oblivious-transfer protocol that is vulnerable to input dependent abort by a malicious sender to full security. The basic idea is to use a special kind of "verifiable" secret sharing that will allow the receiver to open a subset of the shares of both the sender inputs to verify the validity of the shares and input consistency. Only if the checks pass the receiver proceeds to obtain its real output. This extra step helps prevent input dependent abort as if the validity checks pass then with high probability we can reconstruct unique values for both inputs of the sender from the shares. In our protocol, we will implicitly perform the cut-andchoose by relying on the OT protocol itself. We remark that while the issue that needed to be resolved was an input-dependent abort in [55], in our case, we use it to boost the extraction probability of sender's inputs while maintaining the privacy against the receiver. The secret sharing ensures that the receiver cannot learn more than one output and extracting a significant fraction of shares is sufficient to extract the outputs. Another advantage of relying on the OT protocol to perform the cut-and-choose is that the sender needs to use its input only in the third-round of our protocol after the receiver submits its input for the OT instance.

We begin with the following building blocks used in our construction: let (1) Commit be a statistically binding commitment scheme, (2) let (Share, Rec) be a $(M+1)$-out-of$2 M$ Shamir secret-sharing scheme over $\mathbb{Z}_{q}$, together with a linear map $\phi: \mathbb{Z}_{q}^{2 M} \rightarrow \mathbb{Z}_{q}^{M-1}$ such that $\phi(v)=0$ iff $v$ is a valid sharing of some secret. We further note that the WI$\operatorname{PoK} \pi_{\mathrm{DL}}^{\mathrm{WI}}$ that is given by Rec in Protocol 3, is extended here to handle the parallel case. Namely, the receiver proves the validity of one of the public keys it generates within each pair, in parallel. On a high-level, we modify Protocol 3 as follows.

- We repeat Protocol 3 in parallel $3 M$ times to obtain $3 M$ oblivious-transfer parallel executions. We divide this set of executions into two sets of $M$ and $2 M$ executions.

- The sender chooses first two random inputs $x_{0}, x_{1} \in \mathbb{Z}_{q}$ and secret shares them using the Shamir secret-sharing scheme to obtain shares $\left[x_{0}\right]$ and $\left[x_{1}\right]$. Next, for $b \in\{0,1\}$ it picks $M$ pairs of vectors that add up to $\left[x_{b}\right]$. It is instructive to view them as matrices $A_{0}, B_{0}, A_{1}, B_{1} \in \mathbb{Z}_{q}^{M \times 2 M}$ where for every row $i \in[M]$ and $b \in\{0,1\}$, it holds that $A_{b}[i, \cdot] \oplus B_{b}[i, \cdot]=\left[x_{b}\right]$. Next, the sender commits to each entry of each matrix separately in the third message of the protocol. To check the validity of the shares the sender additionally sends matrices $Z_{0}, Z_{1}$ in the clear, such that the row $Z_{b}[i, \cdot]$ is set to $\phi\left(A_{b}[i, \cdot]\right)$, along with the third message of the protocol where it commits to the entries of $A_{0}, A_{1}, B_{0}$ and $B_{1}$. Finally, it sends $C_{0}=x_{0}+s_{0}$ and $C_{1}=x_{1}+s_{1}$. 
- In the first set of $M$ OT executions, the sender's input to the $i$ th execution is the decommitment information of the entire $i$ th row

$$
\left(\left(A_{0}[i, \cdot], A_{1}[i, \cdot]\right),\left(B_{0}[i, \cdot], B_{1}[i, \cdot]\right)\right)
$$

whereas the receiver sets its input to these executions as $c_{1}, \ldots, c_{M}$ at random. Upon receiving its output for the OT, the receiver proceeds as follows: If $c_{i}=0$, then the receiver checks whether $\phi\left(A_{b}[i, \cdot]\right)=\left[z_{i}^{b}\right]$, and if $c_{i}=1$ it checks whether $\phi\left(B_{b}[i, \cdot]\right)+Z_{b}[i, \cdot]=0$. This is referred to as the shares validity check.

- In the second set of $2 M$ OT executions, the sender's input to the $j$ th OT execution is the decommitment information of the entire $j$ th column

$$
\left(\left(A_{0}[\cdot, j], B_{0}[\cdot, j]\right),\left(A_{1}[\cdot, j], B_{1}[\cdot, j]\right)\right) .
$$

Looking ahead, if the receiver's input is $b$, then upon receiving its output for a particular column $j$ it checks that for all $i, A_{b}[i, j] \oplus B_{b}[i, j]$ agree on the same value. We refer to this as the shares consistency check.

- In the second set of OTs, the receiver sets its input as follows. It selects a random subset $T_{1-b} \subseteq[2 M]$ of size $M / 2$ and defines $T_{b}=[2 M] / T_{1-b}$. Then, for every $j \in[2 M]$, Rec sets $b_{j}=\beta$ if $j \in T_{\beta}$. The $b_{j}$ values serve as the inputs to the OT for the next $2 M$ executions.

- Finally, the receiver first checks if all the rows obtained from the first set of OT executions pass the shares validity check. Next, it checks if all the columns in $T_{1-b}$ and a random subset of size $M / 2$ from $T_{b}$ pass the shares consistency check. If so, it finds $M+1$ columns in $T_{b}$ that pass the shares consistency check, extracts the share that corresponds to each such column and then uses these $M+1$ shares to reconstruct $x_{b}$. Finally, the receiver uses $x_{b}$ and $C_{b}$ to compute $s_{b}$.

- Additionally, we modify the WI-PoK to a proof for a statement that captures all parallel executions simultaneously, i.e., the statements of all OT executions are combined using the logical AND. Furthermore, a party rejects if the other party aborts or delivers an incorrect or invalid message.

The security guarantees of this protocol are $1 / p$-security against malicious senders and full security against non-aborting receivers. We remark that the receiver's simulation essentially follows a similar approach as in the simulation of Protocol 3, where it rewinds the WI-PoK protocol in order to extract the receiver's inputs to all the parallel OT executions and then setting the input that the receiver cannot obtain to a random string (one at a time), concluding that there will not be enough information for any receiver to extract $s_{1-b}$. On the other hand, the sender simulation needs to achieve $1 / p$-simulation. The high-level idea is to apply techniques from the simulation in [55], given that the simulator extracts sufficiently enough shares of the sender's inputs to the parallel OTs. The core of our argument and the main technical part of this protocol is to show that if an adversarial sender does not abort before sending the third message too often (i.e., $<1-\frac{1}{p}$ ) then the simulator can extract the trapdoor by rewinding sufficiently many times. Namely, in this case, we show that the simulator can extract the discrete logarithm of both $h_{0}$ and $h_{1}$ with respect to $g$ in at least $1-\frac{1}{3 p}$ fraction of the OT executions. 
Then we can show that the simulator succeeds in extracting the sender's inputs $s_{0}, s_{1}$ with very high probability.

5.1. 4-Round OT with 1/p Sender Security and Full Security Against Non-aborting Receivers

We construct a 4-round OT protocol with the stronger guarantee of $1 / p$-security in the presence of (possibly aborting) malicious senders.

Protocol 4. (Protocol $\pi_{\mathrm{OT}}$ )

Public Parameters The description of a group $\mathbb{G}$ of prime order $q$.

Inputs The sender Sen holds $s_{0}, s_{1}$ and the receiver Rec holds a bit $b$.

\section{The protocol:}

1. Sen $\rightarrow$ Rec

(a) Let $N=3 M$. Then, for $i \in[N]$, Sen picks random generator $g_{i} \leftarrow \mathbb{G}$ and computes $h_{i, 0}=g_{i}^{r_{i, 0}}$ and $h_{i, 1}=g_{i}^{r_{i, 1}}$ where $r_{i, 0}, r_{i, 1} \leftarrow \mathbb{Z}_{q}$.

(b) Sen sends the $N$ tuples $\left\{g_{i}, h_{i, 0}, h_{i, 1}\right\}_{i \in[N]}$ to Rec.

\section{2. $\operatorname{Rec} \rightarrow$ Sen}

(a) Rec samples uniformly at random $c_{1}, \ldots, c_{M} \leftarrow\{0,1\}$. The $c_{i}$ values serve as the input to the first $M O T$ executions.

(b) Rec selects a random subset $T_{1-b} \subseteq[2 M]$ of size $M / 2$. Define $T_{b}=[2 M] / T_{1-b}$. For every $j \in[2 M]$, Rec sets $b_{j}=\alpha$ if $j \in T_{\alpha}$. The $b_{j}$ values serve as the inputs to the OT for the next $2 M$ executions.

(c) According to its input for the $3 M$ OT executions, Rec generates $N=3 M$ pairs of El Gamal PKE's as follows:

- For every $i \in[M], \mathrm{PK}_{i, c_{i}}=g_{i}^{m_{i}}$ and $\mathrm{PK}_{i, 1-c_{i}}=\left(h_{i, 0} h_{i, 1}\right)^{\tilde{m}_{i}}$ where $m_{i}, \tilde{m}_{i} \leftarrow \mathbb{Z}_{q}$. Rec sets $\mathrm{SK}_{i}=m_{i}$.

- For every $j \in[2 M], \mathrm{PK}_{M+j, b_{j}}=g_{M+j}^{m_{M+j}}$ and $\mathrm{PK}_{M+j, 1-b_{j}}=\left(h_{M+j, 0}\right.$ $\left.h_{M+j, 1}\right)^{\tilde{m}_{M+j}}$ where $m_{M+j}, \tilde{m}_{M+j} \leftarrow \mathbb{Z}_{q}$. Rec sets $\mathrm{SK}_{M+j}=m_{M+j}$.

(d) Rec sends $\left\{\mathrm{PK}_{i, 0}, \mathrm{PK}_{i, 1}\right\}_{i \in[N]}$ to Sen.

(e) Rec sends the first message of the WI-PoK for proving the knowledge for every $i \in[N]$ of the discrete logarithms of either $\mathrm{PK}_{0}^{i}$ or $\mathrm{PK}_{1}^{i}$ with respect to $\left(h_{i, 0} h_{i, 1}\right)$.

(f) Rec sends a challenge string $\beta=\left(\beta_{1}, \ldots, \beta_{N}\right)$.

(g) Rec sends the first message for the statistically binding commitment scheme com.

\section{Sen $\rightarrow$ Rec}

(a) Sen picks two random strings $x_{0}, x_{1} \leftarrow \mathbb{Z}_{q}$ and secret shares them using the Shamir's secret-sharing scheme. In particular, Sen computes $\left[x_{b}\right]=\left(x_{b}^{1}, \ldots\right.$, $\left.x_{b}^{2 M}\right) \leftarrow$ Share $\left(x_{b}\right)$ for $b \in\{0,1\}$. Sen commits to the shares $\left[x_{0}\right],\left[x_{1}\right]$ as follows. It picks random matrices $A_{0}, B_{0} \leftarrow \mathbb{Z}_{q}^{M \times 2 M}$ and $A_{1}, B_{1} \leftarrow \mathbb{Z}_{q}^{M \times 2 M}$ such that $\forall i \in[M]$ : 


$$
A_{0}[i, \cdot]+B_{0}[i, \cdot]=\left[x_{0}\right], \quad A_{1}[i, \cdot]+B_{1}[i, \cdot]=\left[x_{1}\right] .
$$

Sen computes two matrices $Z_{0}, Z_{1} \in \mathbb{Z}_{q}^{M \times M-1}$ and sends them in the clear such that:

$$
Z_{0}[i, \cdot]=\phi\left(A_{0}[i, \cdot]\right), Z_{1}[i, \cdot]=\phi\left(A_{1}[i, \cdot]\right)
$$

(b) Sen sends the committed matrices $\left(\mathrm{com}_{A_{0}}, \mathrm{com}_{B_{0}}, \mathrm{com}_{A_{1}}, \mathrm{com}_{B_{1}}\right)$ to $\mathrm{Rec}$ where each element of each matrix is individually committed using com.

(c) For $i \in[M]$, Sen computes ciphertexts $c_{i, 0}, c_{i, 1}$ where $c_{i, 0}$ is an encryption of the decommitment of the rows $A_{0}[i, \cdot]$ and $A_{1}[i, \cdot]$ under public key $\mathrm{PK}_{i, 0}$ and $c_{i, 1}$ is an encryption of the decommitment of the rows $B_{0}[i, \cdot]$ and $B_{1}[i, \cdot]$ under public key $\mathrm{PK}_{i, 1}$. Sen sends $\left\{c_{i, 0}, c_{i, 1}\right\}_{i \in[M]}$ to Rec.

(d) For $j \in[2 M]$, Sen computes ciphertexts $\tilde{c}_{j, 0}, \tilde{c}_{j, 1}$, where $\tilde{c}_{j, b}$ is an encryption of the decommitment of the columns $A_{b}[\cdot, j], B_{b}[\cdot, j]$ under public key $\mathrm{PK}_{M+j, b}$. Sen sends $\left\{\tilde{c}_{j, 0}, \tilde{c}_{j, 1}\right\}_{j \in[2 M]}$ to Rec.

(e) Sen sends the second message $e_{\mathrm{Sen}}$ for the WI-PoK protocol $\pi_{\mathrm{DL}}^{\mathrm{WI}}$ given by the receiver (recall that this message is a random challenge).

(f) Sen sends $r_{\beta_{i}}=\log _{g_{i}}\left(h_{i, \beta}\right)$ for all $i \in[N]$.

(g) Sen sends $C_{0}=s_{0} \oplus x_{0}$ and $C_{1}=s_{1} \oplus x_{1}$ to Rec.

\section{4. $\operatorname{Rec} \rightarrow$ Sen}

(a) Decryption Phase Upon receiving the all the sender's ciphertexts the receiver decrypts them to obtain the OT outputs. These include decommitments to $A_{0}[i, \cdot], A_{1}[i, \cdot]$ for every $i \in[M]$ when $c_{i}=0$ and decommitments to $B_{0}[i, \cdot], B_{1}[i, \cdot]$ when $c_{i}=1$. They also include columns $A_{b_{j}}[\cdot, j], B_{b_{j}}[\cdot, j]$ for every $j \in[2 M]$.

(b) Shares Validity Check Phase For $i=1, \ldots, M$, if $c_{i}=0$ check that $Z_{0}[i, \cdot]=\phi\left(A_{0}[i, \cdot]\right)$ and $Z_{1}[i, \cdot]=\phi\left(A_{1}[i, \cdot]\right)$. Otherwise, if $c_{i}=1$ check that $\phi\left(B_{0}[i, \cdot]\right)+Z_{0}[i, \cdot]=0$ and $\phi\left(B_{1}[i, \cdot]\right)+Z_{1}[i, \cdot]=0$. If all the checks pass, the receiver proceeds to the next phase and otherwise aborts.

(c) Shares Consistency Check Phase For each $b \in\{0,1\}$, Rec randomly chooses a set $T_{b}$ for which $b_{j}=b$ at $M / 2$ coordinates. For each $j \in T_{b}$, Rec checks that there exists a unique $x_{b}^{i}$ such that $A_{b}[i, j]+B_{b}[i, j]=x_{b}^{j}$ for all $i \in[M]$. If so, $x_{b}^{j}$ is marked as consistent. If all shares obtained in this phase are consistent, Rec proceeds to the reconstruction phase. Else it aborts.

(d) Reconstruction Phase For $j \in[2 M] / T_{1-b}$, if there exists a unique $x_{b}^{j}$ such that $A_{b}[i, j]+B_{b}[i, j]=x_{b}^{j}$, Rec marks share $j$ as a consistent column. If $R$ obtains less than $M+1$ consistent columns, it aborts. Otherwise, let $x_{b}^{j_{1}}, \ldots, x_{b}^{j_{M+1}}$ be any set of $M+1$ shares obtained from consistent columns. $\operatorname{Rec}$ computes $x_{b} \leftarrow \operatorname{Reconstruct}\left(x_{b}^{j_{1}}, \ldots, x_{b}^{j_{M+1}}\right)$ and outputs $s_{b}=C_{b} \oplus x_{b}$.

(e) Rec sends the last message for the WI-PoK protocol $\pi_{\mathrm{DL}}^{\mathrm{WI}}$.

Theorem 5.1. Assume that the Decisional Diffie-Hellman assumption holds in $\mathbb{G}$ and that $\pi_{\mathrm{DL}}^{\mathrm{WI}}$ is as above. Then Protocol 4 is a 4-round protocol, where the receiver receives 
the output in the third round, that securely realizes $\mathcal{F}_{\mathrm{OT}}$ with $1 /$ p-security in the presence of aborting senders and with full security in the presence of non-aborting receivers. Furthermore, the protocol is receiver private according to Definition 2.13.

Proof. We note first that this protocol is receiver private, i.e., private against an (aborting) malicious receiver because just as in the previous section, the receiver's messages can be shown to information theoretically hide its bit. We next consider each corruption case separately.

Sen is corrupted Recall that when the sender is corrupted we need to prove $1 / p$ indistinguishability. More precisely, we need to define a simulator that produces a view of the malicious sender $\mathcal{A}$ while extracting both $s_{0}$ and $s_{1}$, where the view and the value learned by the honest receiver is $1 / p$-indistinguishable from the sender's real view and the receiver's output in a real execution. More precisely, for any probabilistic polynomial-time adversary $\mathcal{A}$ controlling Sen, we define a simulator $\mathcal{S}$ that proceeds as follows:

1. $\mathcal{S}$ invokes $\mathcal{A}$ on its input and randomness of appropriate length.

2. Upon receiving from $\mathcal{A}$ the first message, $\mathcal{S}$ computes the second message honestly with input $b=0$. If $\mathcal{A}$ aborts before sending the third message then $\mathcal{S}$ outputs the view of $\mathcal{A}$ and halts.

3. Otherwise, upon receiving $\mathcal{A}$ 's third message, $\mathcal{S}$ records the set $\left\{r_{\beta_{i}}\right\}_{i \in[N]}$. Next, it stalls the main execution and proceeds to rewind $\mathcal{A}$. Specifically, $\mathcal{S}$ rewinds $\mathcal{A}$ to the second message and proceeds as follows:

- For every $i \in[N]$ and $\gamma \in\{0,1\}, \mathcal{S}$ rewinds $\mathcal{A}$ for $T=N^{4} p$ attempts, where in each such attempt $\mathcal{S}$ supplies a uniformly random second message according to the receiver's strategy with input $b=0$, where $\beta_{i}=\gamma$. In each rewinding, $\mathcal{S}$ collects the correct discrete logarithms within $\mathcal{A}$ 's reply.

4. If upon concluding the rewinding phase $\mathcal{S}$ does not obtain the discrete logarithm of both $h_{i, 0}$ and $h_{i, 1}$ for at least $1-1 / 3 p$ fraction of $i \in[N]$, then it halts outputting fail.

5. Otherwise, let $I \subseteq\{1, \ldots, M\}$ and $J \subseteq\{M+1, \ldots, 3 M\}$ be the sets of indices for which it extracts both the discrete logarithms. We remark that $\mathcal{S}$ does not try to extract the sender's inputs in the first $M$ executions, namely, for indices in $I$. Next, $\mathcal{S}$ rewinds $\mathcal{A}$ back to the second message and for every $j \in J$ generates public keys so that it can extract both the sender's inputs. For all other executions, it follows the honest receiver's strategy corresponding to the input $b=0 . \mathcal{S}$ completes the execution by using the witness corresponding to $b=0$ for the receiver in the WI-PoK. ${ }^{6}$ Upon completion, it performs the share consistency check and the share validity check as the honest receiver would and if either of them fail, then the simulator halts outputting the view of $\mathcal{A}$.

5. Otherwise, it decrypts all ciphertexts for which it knows the corresponding secret keys. For each $b \in\{0,1\}$ and $j \in J$, if there exists a unique $x_{b}^{j}$ such that $A_{b}[i, j]+$ $B_{b}[i, j]=x_{b}^{j}, \mathcal{S}$ marks column $j-M$ as consistent. If it obtains at least $M+1$

\footnotetext{
${ }^{6}$ This is possible because for indices outside $J$ it has the correct witness, and for indices in $J$ it has witnesses corresponding to both inputs of the receiver.
} 
shares for $x_{b}$ from consistent columns it reconstructs $x_{b}$, and then obtains $s_{b}$ from $x_{b}$ and $C_{b}$. If not, it sets $s_{b}=\perp$.

6. Finally, $\mathcal{S}$ forwards $\left(s_{0}, s_{1}\right)$ to $\mathcal{F}_{\text {От }}$ and halts, outputting whatever $\mathcal{A}$ does.

Clearly, $\mathcal{S}$ runs in strict polynomial-time. We next prove the correctness of simulation. On a high-level, the second message in all the rewinding attempts is generated identically to the second message of the real execution and is independent of the bit $b$ that the receiver enters. This follows by repeating Protocol 3 in parallel, for which the indistinguishability argument is similar. Let $s \in \omega(\log n)$. Two cases arise:

1. The abort probability of the sender is higher than $1-\frac{1}{N p}$. In this case, $1 / p$ indistinguishability is achieved directly as the simulation outputs views on which the sender aborts with at least the same probability as such views occur in the real view. Now, since this accounts for a probability mass of at least $1-\frac{1}{N p}>1-\frac{1}{p}$, $1 / p$-indistinguishability follows.

2. The abort probability of the sender is at most $1-\frac{1}{N p}$. In this case by setting $M>s p$, for $s$ being some superlogarithmic function in $n$, we argue that except with negligible probability (roughly, $2^{-O(s)}$ ), the simulator will be able to obtain the discrete logarithms of both $h_{i, 0}$ and $h_{i, 1}$, i.e., the trapdoors, for at least $1-\frac{1}{3 p}$ fraction of the indices $i \in[3 M]$ via rewinding. This is formally proven in Claim 5.1. Just as in the previous protocol, we have that for every index in $\{M+1, \ldots, 3 M\}$ that the simulator obtains a trapdoor, it will be able to extract both of the sender's inputs. Specifically, as $M>s p$, we can conclude that the simulator fails to obtain the trapdoor of at most $\frac{1}{3 p} \times 3 M=s$ indices. This means that among the indices in $\{M+1, \ldots, 3 M\}$ it obtains trapdoors for at least $2 M-s$ indices.

Next, from the shares consistency check we can conclude that with very high probability all but $s$ columns contain shares that are consistent. From the shares validity check, we can conclude that with very high probability there is a single row $i_{b}$ corresponding to each $b \in\{0,1\}$ such that $A_{b}[i, \cdot]+B_{b}[i, \cdot]$ contains valid shares of some secret. Combining these checks, we can conclude that there are at least $2 M-s$ columns that are consistent, i.e., the shared value in each row is the same and therefore must equal $A_{b}\left[i_{b}, \cdot\right]+B_{b}\left[i_{b}, \cdot\right]$. Furthermore, from the statistically binding property of the commitment scheme Commit we can conclude that for any one of these consistent columns, there can be only one value for the shares that can be extracted by both the receiver and the simulator.

In this case, we can now conclude that, using the trapdoors, the simulator obtains at least $2 M-s-s$ shares for both inputs among the consistent columns. Since $M>s p$ we have that $2 M-2 s>M+1$ (for $p>2$ ) and from $M+1$ valid shares it can extract $s_{b}$ for each $b \in\{0,1\}$.

Claim 5.1. We say that $i \in[N]$ is extractable, if $\mathcal{S}$ manages to extract the discrete logarithms of both $h_{i, 0}$ and $h_{i, 1}$ with respect to $g_{i}$. If the adversary $\mathcal{A}$ does not abort before sending the third message with probability at least $\frac{1}{N p}$, then except with negligible probability, at least $1-\frac{1}{3 p}$ fraction of the indices are extractable.

Proof. On a high level, we follow a Yao-type amplification argument [62]. First, we observe that the distribution of the second message fed to $\mathcal{A}$ in any rewinding attempt is perfectly distributed to the real distribution. Next, suppose that $\mathcal{A}$ does not abort 
with probability at least $\frac{1}{N^{3} p}$ both when its view is conditioned on $\beta_{i}=0$ and when it is conditioned on $\beta_{i}=1$, for some index $i$. Then we show that $i$ is extractable except with negligible probability. This is because for every $i$ and every value of $\beta_{i}$ the simulator makes $N^{4} p$ rewinding attempts, thus the probability that it fails to find a successful execution where the adversary $\mathcal{A}$ responds is at most $\left(1-\frac{1}{N^{3} p}\right)^{N^{4} p}=$ $O\left(e^{-s}\right)$. Therefore, it suffices to show that this condition holds for more than $1-\frac{1}{3 p}$ indices $i$ for which $\mathcal{A}$ does not abort with probability at least $\frac{1}{N^{3} p}$ both when its view is conditioned on $\beta_{i}=0$ and when it is conditioned on $\beta_{i}=1$. This is because using the preceding argument, we can conclude that at least $1-\frac{1}{3 p}$ fraction of indices are extractable and the proof of the claim follows.

Suppose for contradiction that there are more than $\frac{1}{3 p}$ fraction of indices for which the condition does not hold. This means that for a set of $\frac{1}{3 p} N=s$ indices, denoted by $S$, and values $\left\{\gamma_{j}\right\}_{j \in S}$ such that for every $j \in S$, when conditioned on $\beta_{j}=\gamma_{j}$, the probability that the adversary aborts is greater than $1-\frac{1}{N^{3} p}$. We now estimate the overall probability that $\mathcal{A}$ does not abort.

$$
\begin{aligned}
\operatorname{Pr}[\mathcal{A} \text { does not abort }]= & \operatorname{Pr}\left[\mathcal{A} \text { does not abort } \mid \exists j \in S \text { s.t. } \beta_{j}=\gamma_{j}\right] \\
& \operatorname{Pr}\left[\exists j \in S \text { s.t. } \beta_{j}=\gamma_{j}\right] \\
& +\operatorname{Pr}\left[\mathcal{A} \text { does not abort } \mid \forall j \in S, \beta_{j} \neq \gamma_{j}\right] \\
& \operatorname{Pr}\left[\forall j \in S, \beta_{i} \neq \gamma_{i}\right] \\
\leq & \left(\sum_{j \in S} \operatorname{Pr}\left[\mathcal{A} \text { does not abort } \mid \beta_{j}=\gamma_{j}\right] \operatorname{Pr}\left[\beta_{j}=\gamma_{j}\right]\right)+1 \\
& \times \operatorname{Pr}\left[\forall j \in S, \beta_{j} \neq \gamma_{j}\right] \\
\leq & \frac{N}{N^{3} p}+\frac{1}{2^{s}} \\
\leq & \frac{1}{2 N^{2} p} .
\end{aligned}
$$

This is a contradiction since we assumed that $\mathcal{A}$ does not abort with probability at least $\frac{1}{N p}$.

Finally, to argue $1 / p$-indistinguishability, we consider two cases:

Case: Non-aborting Probability of $\mathcal{A}$ is Greater than $\frac{1}{p N}$ First, we observe that the sender's view in the simulation is statistically close to the real view. This follows using an argument analogous to Claim 4.1 as the public keys in the second message (even those generated by the simulation using the trapdoors) and the perfect WI-PoK perfectly hide the receiver's input. It therefore suffices to argue that the receiver's messages can be generated while extracting the sender's input. Using the preceding argument, we have that the simulation will always succeed in extracting the trapdoors of at least $1-\frac{1}{3 p}$ fraction of the parallel OT executions. Since $M>s p$, 
we can conclude that the simulator fails to obtain the trapdoor of at most $\frac{1}{3 p} \times 3 M=$ $s$ indices. This means that among the indices in $\{M+1, \ldots, 3 M\}$ it obtains trapdoors for at least $2 M-s$ indices. Recall that, after extraction, the simulator rewinds the sender to the second message and generates the receiver's message by setting up the public keys as follows: for every index in $J$, the simulator uses the trapdoor and sets the public keys so that it can extract both of the sender's inputs. For the rest of the indices, it simply sets the receiver's input bit to 0 .

Next, from the shares consistency check we can conclude that with very high probability all but $s$ columns contain shares that are consistent. Moreover, the share validity check makes the receiver check if $Z_{b}[i, \cdot]=\phi\left(A_{b}[i, \cdot]\right)$ holds or $Z_{b}[i, \cdot]+\phi\left(B_{b}[i, \cdot]\right)=0$ holds. If for a row both conditions hold, then we have the $\phi\left(A_{b}[i, \cdot]\right)+\phi\left(B_{b}[i, \cdot]\right)=0$ and $A_{b}[i, \cdot]+B_{b}[i, \cdot]$ must contain a valid vector of shares. Now even if one of these two conditions fail to hold for more than $s$ rows, the sender will be caught with probability $1-2^{-s}$. Therefore, there are at least $M-s$ rows for which $\phi\left(A_{b}[i, \cdot]\right)+\phi\left(B_{b}[i, \cdot]\right)=0$. For our argument, it suffices to have just one row $i_{b}$ corresponding to each $b \in\{0,1\}$ such that $A_{b}[i, \cdot]+B_{b}[i, \cdot]$ contains valid shares of some secret. Combining these checks, we can conclude that there are at least $2 M-s$ columns that are consistent, i.e., the shared value in each row is the same and must equal $A_{b}\left[i_{b}, \cdot\right]+B_{b}\left[i_{b}, \cdot\right]$. Furthermore, from the statistically binding property of the commitment scheme Commit we can conclude that for any one of these consistent columns, there can be only one value for the shares that can be extracted by both the receiver and the simulator.

In this case, we can now conclude that, using the trapdoors, the simulator obtains at least $2 M-s-s$ shares for both inputs among the consistent columns. Since $M>s p$ we have that $2 M-2 s>M+1$ (for $p>2$ ) and from $M+1$ valid shares it can extract $s_{b}$ for each $b \in\{0,1\}$. Furthermore, the sender's input extracted by the honest receiver while holding the input $b$ and the input extracted by the simulator have to be the same as both of them have to correspond to the shares in the row $i_{b}$.

Case: Non-aborting Probability of $\mathcal{A}$ is at most $\frac{1}{p N}$. From the first step of the simulation, we know that all views on which $\mathcal{A}$ aborts are simulated at least with the same probability as in the real view. Now, if the non-aborting probability is smaller than $\frac{1}{p N}$ then the probability mass of aborting views is at least $1-\frac{1}{p N}>1-\frac{1}{p}$ and we achieve $1 / p$-indistinguishability.

Thus, we have the following claim.

Claim 5.2. The following two distribution ensembles are identical,

$$
\begin{aligned}
& \left\{\operatorname{View}_{\pi_{\mathrm{OT}}, \mathcal{A}(z)}\left(n,\left(s_{0}, s_{1}\right), b\right)\right\}_{n \in \mathbb{N}, s_{0}, s_{1}, b, z \in\{0,1\}^{*}} \\
& \quad \stackrel{1 / \mathrm{p}}{\approx}\left\{\operatorname{View}_{\mathcal{F}_{\mathrm{OT}}, \mathcal{S}(z)}\left(n,\left(s_{0}, s_{1}\right), b\right)\right\}_{n \in \mathbb{N}, s_{0}, s_{1}, b, z \in\{0,1\}^{*}} .
\end{aligned}
$$

Proof. The proof follows essentially using the same ideas from the previous protocol.

Rec is corrupted. In this case, we need to prove that any non-aborting corrupted receiver cannot learn anything about the sender's other input $s_{1-b}$ while extracting $b$. 
More precisely, for any probabilistic polynomial-time adversary $\mathcal{A}$ controlling $\operatorname{Rec}$ we define a simulator $\mathcal{S}$ that proceeds as follows:

1. $\mathcal{S}$ invokes $\mathcal{A}$ on its input and randomness of the appropriate length.

2. $\mathcal{S}$ plays the role of the honest sender with arbitrary inputs $\left(s_{0}^{\prime}, s_{1}^{\prime}\right)$. Upon completing the execution successfully, $\mathcal{S}$ stalls the main execution and proceeds to rewind $\mathcal{A}$. Specifically, $\mathcal{S}$ rewinds $\mathcal{A}$ to the third message and supplies a different second message for $\pi_{\mathrm{DL}}^{\mathrm{WI}}$ by sampling uniformly random new challenge $e_{\mathrm{Sen}}^{\prime}$. If $e_{\mathrm{Sen}}=e_{\mathrm{Sen}}^{\prime}$, i.e., the challenge is identical, then $\mathcal{S}$ aborts. Otherwise, it feeds the challenge to $\mathcal{A}$ as part of the second message. Finally, $\mathcal{S}$ runs the extractor for the WI-PoK $\pi_{\mathrm{DL}}^{\mathrm{WI}}$ and extracts the inputs to all the OT executions along with the discrete logarithm of the corresponding key.

3. Among the executions $M+1, \ldots, 3 M, \mathcal{S}$ finds that bit $b$ that occurs at least $M+1$ times and submits $b$ to $\mathcal{F}_{\text {OT }}$, receiving back $s_{b}$. Recall that since the receiver is a nonaborting adversary, it completes the protocol without allowing the honest sender to abort. In other words, it convinces the sender in the WI-PoK with probability 1. Therefore, since a witness will be extracted from the proof-of-knowledge, the inputs of the receiver in the parallel OTs are well defined. Specifically, $\mathcal{S}$ extracts the adversary's inputs to these OT executions as in the simulation for Protocol 3.

4. $\mathcal{S}$ rewinds $\mathcal{A}$ to the third message and computes it based on $s_{b}$ and random $s_{1-b}$.

5. $\mathcal{S}$ halts, outputting whatever $\mathcal{A}$ does.

Note first that the simulator runs in polynomial-time and that the probability it aborts is negligible. Moreover, we prove that the simulated and real views are computationally indistinguishable via a reduction to the security of the El Gamal PKE. We provide a brief proof sketch below:

- From the proof of Protocol 3, using the privacy argument of the El Gamal PKE, we know that if for a particular OT execution the sender (played by the simulator) extracted the receiver's input as $\gamma$, then the sender's input that corresponds to the bit $1-\gamma$ can be replaced by a random value. We consider a sequence of hybrids where we replace at least one input in each of the $3 M$ executions with a random input. More formally, for every $j \in\{1, \ldots, M\}$, depending on what value is extracted for each of the $c_{i}$, and every $b \in\{0,1\}$, we replace the sender's input containing the decommitment of $A_{b}[i, \cdot]$ with random or that containing the decommitment of $B_{b}[i, \cdot]$ with random. Next, for $j \in\{M+1, \ldots, 3 M\}$ depending on the value extracted as $b_{i}$ for the receiver, we replace the input containing the decommitment of $\left(A_{0}[\cdot, j-M], B_{0}[\cdot, j-M]\right)$ random or the other input containing the decommitment of $\left(A_{1}[\cdot, j-M], B_{1}[\cdot, j-M]\right)$ random. Next, in another sequence of hybrids, for every value that is set to random we also replace the corresponding commitment to a random value.

- Next, we argue that at least $M$ shares of $x_{1-b}$ (out of the $2 M$ shares) are hidden, where $b$ is the adversary's input as extracted in the simulation. To this end, for any column $j$ and row $i$ such that $b_{j} \neq 1-b$, only one of the entries $A_{1-b}[i, j]$ or $B_{1-b}[i, j]$ is revealed (while the other entry is set to random, depending on the choice of $c_{i}$ ). This is because when $b_{j}=b$, the information regarding the $1-b$ th matrices is available only from the rows being revealed. Next, note that $A_{1-b}[i, j], B_{1-b}[i, j]$ are individually distributed uniform at random, therefore 
$A_{1-b}[i, j]+B_{1-b}[i, j]$ is hidden. Now, since $b_{j} \neq 1-b$ for at least $M$ values of $j$ we conclude that at least $M$ shares of $x_{1-b}$ are hidden. Therefore, at most $M$ shares can be recovered but $M$ shares information theoretically hide $x_{1-b}$.

Therefore, we conclude that

Claim 5.3. The following two distribution ensembles are computationally indistinguishable,

$$
\begin{aligned}
& \left\{\mathbf{V i e w}_{\pi_{\mathrm{OT}}, \mathcal{A}(z)}\left(n,\left(s_{0}, s_{1}\right), b\right)\right\}_{n \in \mathbb{N}, s_{0}, s_{1}, b, z \in\{0,1\}^{*}} \\
& \quad \stackrel{\mathrm{c}}{\approx}\left\{\operatorname{View}_{\mathcal{F}_{\mathrm{OT}}, \mathcal{S}(z)}\left(n,\left(s_{0}, s_{1}\right), b\right)\right\}_{n \in \mathbb{N}, s_{0}, s_{1}, b, z \in\{0,1\}^{*}} \cdot
\end{aligned}
$$

As a final remark, we note that Protocol 4 can be viewed as a 3-round protocol by removing the WI-PoK given by the receiver. This implies that we can remove the last round sent by the receiver. Then the security guarantee of the modified protocol is the same with respect to malicious senders, whereas security against malicious receivers is ensured in the presence of defensible private adversaries (cf. Definition 2.7.1). Intuitively, the proof follows due to the following argument. If a malicious receiver is able to provide a valid defense, which includes an input and randomness, this implies that for each pair of keys it provides a discrete logarithm with respect to $h_{i, 0}, h_{i, 1}$. Then, a reduction to the privacy of El Gamal can be constructed similarly by reducing the distinguishing probability between the two views to the distinguishing probability between two ciphertexts.

\subsection{4-Round $2 P C$ with 1/p Sender Security and Full Security Against Non-aborting Receivers}

Obtaining general secure two-party computation is carried out analogous to Protocol 3 by embedding the 2-round protocol of [43] within the second/third messages of our OT protocol. It follows just as in Sect. 4.3 that we obtain a two-party protocol that is secure against malicious non-aborting adversaries. More concretely, we have the following theorem:

Theorem 5.2. Assuming the Decisional Diffie-Hellman problem is hard, there exists a 4-round two-party secure protocol for any functionality, where both parties receive the output, that is $1 /$ p-secure in the presence of aborting senders and fully secure in the presence of non-aborting receivers.

Proof Sketch. Recall that, in our previous protocol, to achieve simulation when the receiver is corrupted, we consider a simulator that honestly generates the sender's messages with arbitrary inputs for the functionality being computed and then extracts the receiver's inputs to the OT by rewinding the WI-PoK. By relying on precisely the same strategy, we can obtain the receiver's inputs in this protocol and then complete the simulation by relying on the simulator for the malicious receiver in [43] protocol. 
To achieve simulation when the sender is corrupted, we combine the following two observations:

- First, using the approach from our previous protocol, it follows that whenever the simulator extracts the required trapdoor, it is possible to generate the OT part in the second message from the receiver in a way that it is identically distributed to the real receiver's message while at the same time extracting the sender's inputs to the OT. Furthermore, whenever the extraction of the sender's inputs is successful, we can rely on the simulation of [43] in the $\mathcal{F}_{\mathrm{OT}}$-hybrid to complete the rest of the simulation.

- Second, we observe that, if the sender aborts before sending the third message, no extraction is needed to be carried out since no inputs need to be feed to the $\mathcal{F}_{\text {OT }}$-functionality.

We can now conclude that our simulation achieves $1 / p$-security against malicious senders, by using the same two cases as we considered for Protocol 4 based on the abort probability of the sender. More precisely,

Case: Non-aborting Probability of $\mathcal{A}$ is Greater than $\frac{1}{p N}$ In this case, we know that except with probability $O\left(\frac{1}{p}\right)$ the simulator extracts the required trapdoors and we achieve perfect simulation with probability at least $1-O\left(\frac{1}{p}\right)$.

Case: Non-aborting Probability of $\mathcal{A}$ is at most $\frac{1}{p N}$ If the non-aborting probability is smaller than $\frac{1}{p N}$ then the probability mass of aborting views is at least $1-\frac{1}{p N}>1-\frac{1}{p}$ and since no extraction needs to be carried out we achieve $1 / p$-security.

\section{3-Round OT with 1/p Sender Security and Receiver Privacy}

In this section, we construct a three-round protocol that additionally achieves receiver privacy while maintaining privacy and $1 / p$-security against malicious senders. In contrast to the previous construction which relied on the discrete logarithm assumption, this construction is based on claw-free (trapdoor) permutations. We begin with a description of a warmup 3-round oblivious-transfer protocol that only provides receiver privacy, and then, relying on the techniques from Section 5, we discuss how to achieve $1 / p$-security against aborting senders. Privacy against malicious senders will directly follow from the fact that the receiver's message statistically hides its input due to the clew-freeness of the underling function.

We recall first the definition of claw-free trapdoor permutations. Our definition is slightly more restrictive in that we require both functions in every pair to be permutations and invertible with a trapdoor. We note that the RSA-based claw-free permutations collection satisfies this definition [33].

Definition 6.1. A collection of functions $\left\{\left(f_{i}^{0}: D_{i} \rightarrow D_{i}, f_{i}^{1}: D_{i} \rightarrow D_{i}\right)\right\}_{i \in I}$ for an index set $I \subset\{0,1\}^{*}$ is a family of claw-free permutations if the following holds: 
- There exists a PPT algorithm Gen that on input $1^{n}$ outputs a random index $i \in$ $I \cap\{0,1\}^{n}$ and a trapdoor information $\mathrm{tk}^{0}, \mathrm{tk}^{1}$.

- There exists efficient sampling algorithms which, on input $i$, outputs a random element $x \in D_{i}$.

- Each function $f_{i}^{0}$ and $f_{i}^{1}$ are efficiently computable given $i$ and input $x \in D_{i}$.

- For every $i, f_{i}^{b}$ is a permutation and is efficiently invertible given the trapdoor information $\mathrm{tk}^{b}$.

- For any PPT algorithm $B$, there exists a negligible function $\epsilon(\cdot)$ such that

$$
\forall n, \operatorname{Pr}\left[\left(i, \mathrm{tk}^{0}, \mathrm{tk}^{1}\right) \leftarrow \operatorname{Gen}\left(1^{n}\right) ;\left(x_{0}, x_{1}\right) \leftarrow B(i): f_{i}^{0}\left(x_{0}\right)=f_{i}^{1}\left(x_{1}\right)\right] \leq \epsilon(n) .
$$

Next, we describe our warmup protocol for achieving privacy in the presence of aborting adversaries.

\section{Protocol 5. (Protocol $\pi_{\mathrm{OT}}$ )}

Inputs The sender Sen holds $s_{0}, s_{1}$ and the receiver Rec holds a bit $b$.

\section{The protocol:}

1. Sen $\rightarrow$ Rec Sen samples $\left(i, \mathrm{tk}^{0}, \mathrm{tk}^{1}\right) \leftarrow \operatorname{Gen}\left(1^{n}\right)$ and sends $i$ to the receiver $\mathrm{Rec}$.

2. $\operatorname{Rec} \rightarrow$ Sen Rec samples $x \leftarrow D_{i}$ and sends $y=f_{i}^{b}(x)$.

3. Sen $\rightarrow$ Rec Upon receiving $y$, Sen computes $x_{\beta}=\left(f_{i}^{\beta}\right)^{-1}(y)$ for all $\beta \in\{0,1\}$, and sends $\left(\left\langle x_{0}, r_{0}\right\rangle \oplus s_{0}, r_{0}\right)$ and $\left(\left\langle x_{1}, r_{1}\right\rangle \oplus s_{1}, r_{1}\right)$ for random $r_{0}, r_{1}{ }^{7}$

Theorem 6.2. Assume the existence of claw-free trapdoor permutations. Then, Protocol 5 is a three-round protocol that securely realizes $\mathcal{F}_{\text {От }}$ with privacy in the presence of aborting receivers and senders.

Proof. We will argue privacy against a malicious receiver and malicious sender separately.

Privacy Against a Malicious Sender Receiver's privacy follows directly from the fact that the receiver's bit is information theoretically hidden, as the receiver's message $y$ is uniformly distributed over $D_{i}$ and independent of its input. Namely, it is possible to invert $y$ with respect to both $f_{i}^{0}$ and $f_{i}^{1}$.

Privacy Against a Malicious Receiver We will prove that this protocol guarantees privacy against a malicious receiver according to Definition 2.12. Assume for contradiction, there exists a malicious receiver $\operatorname{Rec}^{*}$, PPT distinguisher $D$, polynomial $p(\cdot)$, two tuples $\left(s_{0}^{0}, s_{1}^{0}, z_{0}\right)$ and $\left(s_{0}^{1}, s_{1}^{1}, z_{1}\right)$ such that with probability at least $\frac{1}{p(n)}$ over $\operatorname{PExec}_{\operatorname{Rec}}\left(1^{n}\right)=\left(\left(m_{s}^{1}, m_{r}\right), \sigma, r_{\operatorname{Rec}}\right)$, it holds for both $b=0$ and $b=1$ that

$$
\begin{aligned}
& \mid \operatorname{Pr}\left[m_{s}^{2} \leftarrow \operatorname{Sen}_{2}\left(\sigma, m_{r},\left(s_{0}^{b}, s_{1}^{b}\right)\right): D\left(1^{n}, z_{b}, r_{\operatorname{Rec}},\left(m_{s}^{1}, m_{s}^{2}\right)\right)=1\right] \\
& \quad-\mid \operatorname{Pr}\left[s_{b}^{*} \leftarrow s_{b}^{b} ; s_{1-b}^{*} \leftarrow\{0,1\}^{\ell(n)} ; m_{s}^{2} \leftarrow \operatorname{Sen}_{2}\left(\sigma, m_{r},\left(s_{0}^{*}, s_{1}^{*}\right):\right.\right.
\end{aligned}
$$

\footnotetext{
${ }^{7}$ We can consider some canonical representation of elements in $D_{i}$ in $\{0,1\}^{*}$.
} 


$$
D\left(1^{n}, z_{b}, r_{\operatorname{Rec}},\left(m_{s}^{1}, m_{s}^{2}\right)\right)=1 \mid \geq \frac{1}{p(n)}
$$

Now, we are ready to prove this theorem by reducing such an adversary to breaking the claw-freeness. For this discussion, we will assume that the sender's inputs are all bits. On a high level, the idea is that if there exists such a distinguisher that can observe when the input corresponding to bit $b$ is replaced by a random bit, then using the Goldreich-Levin theorem it is possible to extract an inverse of $y$ under $f_{b}$ in the protocol, call it $x_{b}$. Then if we can obtain both $x_{0}$ and $x_{1}$ we obtain a claw and arrive at a contradiction.

Consider an adversary $\mathcal{A}^{*}$ that on input an index $i \leftarrow \operatorname{Gen}\left(1^{n}\right)$ from the claw-free family proceeds as follows:

- It starts the emulation against $\operatorname{Rec}^{*}$ by supplying $m_{s}^{1}=i$ as the first message of the sender. Next, it stalls the execution after Rec* produces $m_{r}=y$. Let the state of the partial execution be $\operatorname{PExec}_{\operatorname{Rec}}\left(1^{n}\right)=\left(\left(m_{s}^{1}, m_{r}\right), \sigma, r_{\operatorname{Rec}}\right)$.

- For each $b \in\{0,1\}, \mathcal{A}^{*}$ proceed as follows:

1. $\mathcal{A}^{*}$ needs to generate the third message according to the protocol and feed it to $D$. Recall that the third message is obtained by computing $x_{0}=\left(f_{i}^{0}\right)^{-1}(y)$ and $x_{1}=\left(f_{i}^{1}\right)^{-1}(y)$ and using its inputs $s_{0}$ and $s_{1}$ to compute $\left(\left\langle x_{0}, r_{0}\right\rangle \oplus s_{0}, r_{0}\right)$ and $\left(\left\langle x_{1}, r_{1}\right\rangle \oplus s_{1}, r_{1}\right)$ for random $r_{0}, r_{1}$. Moreover, $\mathcal{A}^{*}$ does not have the trapdoors for the functions. Instead, $\mathcal{A}^{*}$ creates a predictor algorithm $P_{b}^{*}$ that on input $r_{b}$ tries to predict the value of $\left\langle x_{b}, r_{b}\right\rangle$ where $x_{b}=\left(f_{i}^{b}\right)^{-1}(y)$. The idea is that if it can obtain a good predictor, it can invoke the Goldreich-Levin theorem. More precisely, $P_{b}^{*}$ is a machine that has values $\left(r_{1-b}, t_{1-b}, m_{s}^{1}=i, m_{r}=y, r_{\mathrm{Rec}}\right)$ hardcoded and internally emulates the distinguisher $D$ as follows: On input $r_{b}$, it sets the third message $m_{s}^{2}$ as $\left(u, r_{b}\right)$ and $\left(t_{1-b}, r_{1-b}\right)$ where $u$ is a random bit. Then it runs the distinguisher $D$ on input $\left(1^{n}, z_{b}, r_{\operatorname{Rec}},\left(m_{s}^{1}, m_{s}^{2}\right)\right.$. If $D$ outputs $1,{ }^{8}$ then $P_{b}^{*}$ outputs $u$; otherwise, it outputs $1-u$.

2. $\mathcal{A}^{*}$ next runs the Goldreich-Levin extractor algorithm as follows. It samples a random string $r_{1-b}$ and bit $t_{1-b}$ and runs extractor on $P_{b}^{*}\left(r_{1-b}, t_{1-b}, i, y, r_{\operatorname{Rec}}\right)$. If the extractor algorithm outputs a valid $x_{b}$, then $\mathcal{A}^{*}$ collects $x_{b}$.

- If $\mathcal{A}^{*}$ obtains valid $x_{0}$ and $x_{1}$, it outputs them and halts. Otherwise it aborts.

We now analyze the success probability of $\mathcal{A}^{*}$. We show that with probability $\frac{1}{\operatorname{poly}(q, n)}$ it extracts both $x_{0}$ and $x_{1}$. Consider the following events:

Event 1 Conditioned on the partial execution $\operatorname{PExec}_{\operatorname{Rec}^{*}}\left(1^{n}\right)=\left(\left(m_{s}^{1}, m_{r}\right), \sigma, r_{\operatorname{Rec}}\right)$, Equation 1 holds.

Event 2 The bit chosen for $t_{1-b}$ is equal to $\left\langle x_{1-b}, r_{1-b}\right\rangle \oplus s_{1-b}^{b}$.

If these events occur, then except with negligible $P_{b}^{*}\left(r_{1-b}, t_{1-b}, i, y, r_{\mathrm{Rec}}\right)$ is a good predictor, namely it can guess $\left\langle x_{b}, r_{b}\right\rangle$ with probability non-negligible better than a half. Then, by the Goldreich-Levin theorem $\mathcal{A}^{*}$ extracts $x_{b}$ with probability at least $\frac{1}{\text { poly }(n, p(n))}$. Since $\mathcal{A}^{*}$ can extract both $x_{0}$ and $x_{1}$ with non-negligible probability, we conclude that $\mathcal{A}^{*}$ violates the claw-freeness of the function family. It therefore suffices to show that the Events 1 and 2 occur with non-negligible probability. Event 1 occurs

\footnotetext{
${ }^{8}$ We assume without loss of generality that $D$ outputs 1 with higher probability in the game Game . $_{\text {. }}$
} 
with probability $\frac{1}{p(n)}$ by our assumption. Event 2 occurs with each bit $b$ with probability $\frac{1}{2}$ and therefore occurs for both bits with probability at least $\frac{1}{4}$. Overall the probability both events occur is at least $\frac{1}{4 p(n)}$ and this concludes the proof of receiver privacy.

Toward 1/p-Simulation of a Malicious Sender Next, we make the observation that to achieve sender simulation, we need a mechanism to extract the sender's input while maintaining the receiver's message distribution. This can be achieved if the simulator $\mathrm{knows} \mathrm{tk}^{b}$ for at least one value of $b$. With $\mathrm{tk}^{b}$, the simulator can sample $x_{1-b}$ at random and compute $x_{b}=\left(f_{i}^{b}\right)^{-1}(y)$ using $\mathrm{tk}^{b}$ where $y=f_{i}^{1-b}\left(x_{1-b}\right)$. Now, the simulator supplies this $y$ as the input and using both $x_{0}$ and $x_{1}$ extracts both $s_{0}$ and $s_{1}$. Since $y$ is distributed identically as the real distribution we achieve simulation. Hence, there is a trapdoor information that allows simulation which is committed to by the sender in the first message via the function index $i$.

To achieve $1 / p$-simulation against an aborting sender, we repeat our basic protocol in parallel analogous to Protocol 4 where we rely on the OT protocol to perform the cutand-choose checks. In slight more detail, we modify the sender's algorithm analogously to also commit to its input by appropriately secret sharing its input. After the sender sends the first message, the receiver picks a subset of size $3 M$ and sends the indices. For the remaining $3 M$ indices, the receiver sets its input according to the previous protocol. The sender reveals the trapdoors for the indices requested by the receiver, and the for the unopened indices, it sends its OT inputs according to the previous protocol by secret sharing.

To argue receiver privacy, we observe that receiver privacy composes in parallel just as witness indistinguishability does and therefore the receiver will not be able to learn at least one of the two inputs in all parallel executions. Privacy then holds from following an argument analogous to our previous protocol where we show that receiver can learn sufficiently many shares for only one of the two sender's inputs. Achieving $1 / p$ sender simulation, on the other hand, follows using a standard cut-and-choose argument to establish that, through rewinding, a simulator can extract sufficiently many trapdoors as long as the sender does not abort too often. In fact, from Claim 5.1 it follows that it will obtain all but $O(s)$ of the trapdoors for the case where the sender does not abort too often. With these trapdoors, the same strategy as the previous protocol can be carried out here. This protocol additionally achieves full simulation against non-aborting senders. A complete proof is provided below.

\section{Protocol 6. (Protocol $\pi_{\mathrm{OT}}$ )}

Inputs The sender Sen holds $s_{0}, s_{1}$ and the receiver Rec holds $a$ bit $b$.

\section{The protocol:}

- Sen $\rightarrow \operatorname{Rec}$ Let $N=6 M$. Then, for $j \in[N]$, Sen samples $\left(i n d_{j}, \mathrm{tk}_{j}^{0}, \mathrm{tk}_{j}^{1}\right) \leftarrow$ Gen $\left(1^{n}\right)$ and sends ind $1, \ldots$, ind $d_{N}$ to the receiver $\operatorname{Rec}$.

- $\operatorname{Rec} \rightarrow$ Sen Rec picks a subset Trap $\subset[N]$ of size $N / 2$ and sends Trap to Sen. Let the remaining $3 M$ indices be $\left\{a_{1}, \ldots, a_{3 M}\right\}$. For these indices, the receiver proceeds as follows

1. Rec samples uniformly at random $c_{1}, \ldots, c_{M} \leftarrow\{0,1\}$. The $c_{i}$ values serve as the input to the first M OT executions. 
2. Rec selects a random subset $T_{1-b} \subseteq[2 M]$ of size $M / 2$. Define $T_{b}=[2 M] / T_{1-b}$. For every $j \in[2 M]$, Rec sets $b_{j}=\alpha$ if $j \in T_{\alpha}$. The $b_{j}$ values serve as the inputs to the OT for the next $2 M$ executions.

3. According to its input for the $3 M$ OT executions, Rec generates image elements as follows:

- For every $i \in[M]$, it samples $x_{j} \leftarrow D_{a_{i}}$, and sends $y_{j}=f_{a_{i}}^{c_{i}}\left(x_{j}\right)$.

- For every $j \in[2 M]$, it samples $x_{M+j} \leftarrow D_{a_{M+j}}$, and sends $y_{M+j}=$ $f_{a_{M+j}}^{b_{j}}\left(x_{M+j}\right)$.

- Sen $\rightarrow$ Rec

1. Upon receiving Trap and $y_{1}, \ldots, y_{3 M}$, Sen sends $\mathrm{tk}_{j}^{0}$ for all $j \in$ Trap.

2. Sen picks two random strings $t_{0}, t_{1}$ and secret shares them using $(M+1)$-outof-2M Shamir's secret-sharing scheme. In particular, Sen computes $\left[t_{b}\right]=$ $\left(t_{b}^{1}, \ldots, t_{b}^{2 M}\right) \leftarrow$ Share $\left(t_{b}\right)$ for $b \in\{0,1\}$. Sen commits to the shares $\left[t_{0}\right],\left[t_{1}\right]$ as follows. It picks random matrices $A_{0}, B_{0} \leftarrow \mathbb{Z}_{q}^{M \times 2 M}$ and $A_{1}, B_{1} \leftarrow$ $\mathbb{Z}_{q}^{M \times 2 M}$ such that $\forall i \in[M]$ :

$$
A_{0}[i, \cdot]+B_{0}[i, \cdot]=\left[t_{0}\right], \quad A_{1}[i, \cdot]+B_{1}[i, \cdot]=\left[t_{1}\right] .
$$

Sen computes two matrices $Z_{0}, Z_{1} \in \mathbb{Z}_{q}^{M \times M-1}$ and sends them in the clear such that:

$$
Z_{0}[i, \cdot]=\phi\left(A_{0}[i, \cdot]\right), Z_{1}[i, \cdot]=\phi\left(A_{1}[i, \cdot]\right)
$$

3. Sen sends the committed matrices $\left(\mathrm{com}_{A_{0}}, \mathrm{com}_{B_{0}}, \mathrm{com}_{A_{1}}, \mathrm{com}_{B_{1}}\right)$ to $\mathrm{Rec}$ where each element of each matrix is individually committed using com.

4. For $i \in[M]$, Sen computes $x_{\beta}^{i}=\left(f_{a_{i}}^{\beta}\right)^{-1}\left(y_{i}\right)$ for all $\beta \in\{0,1\}$ and sends $\left(\left\langle x_{0}^{i}, r_{0}^{i}\right\rangle \oplus t_{0}^{i}, r_{0}^{i}\right)$ and $\left(\left\langle x_{1}^{i}, r_{1}^{i}\right\rangle \oplus t_{1}^{i}, r_{1}^{i}\right)$ for random $r_{0}^{i}, r_{1}^{i}$.

5. For all $j \in[2 M]$, Sen computes $x_{\beta}^{M+j}=\left(f_{a_{M+j}}^{\beta}\right)^{-1}\left(y_{M+j}\right)$ for all $\beta \in\{0,1\}$ and sends $\left(\left\langle x_{0}^{M+j}, r_{0}^{M+j}\right\rangle \oplus\left(A_{0}[\cdot, j], B_{0}[\cdot, j]\right), r_{0}^{M+j}\right)$ and $\left(\left\langle x_{1}^{M+j}, r_{1}^{M+j}\right\rangle \oplus\right.$ $\left.\left(A_{1}[\cdot, j], B_{1}[\cdot, j]\right), r_{1}^{M+j}\right)$ for random $r_{0}^{M+j}, r_{1}^{M+j}$.

6. Sen sends $C_{0}=s_{0} \oplus t_{0}$ and $C_{1}=s_{1} \oplus t_{1}$ to Rec.

- Rec computes the output of the as follows:

1. Decryption Phase Upon receiving the senders message, the receiver computes the actual OT outputs for all parallel invocations. These include decommitments to $A_{0}[i, \cdot], A_{1}[i, \cdot]$ for every $i \in[M]$ when $c_{i}=0$ and decommitments to $B_{0}[i, \cdot], B_{1}[i, \cdot]$ when $c_{i}=1$. They also include columns $A_{b_{j}}[\cdot, j], B_{b_{j}}[\cdot, j]$ for every $j \in[2 M]$. If any of the decommitments are incorrect, the receiver aborts.

2. Shares Validity Check Phase For $i=1, \ldots, M$, if $c_{i}=0$ check that $Z_{0}[i, \cdot]=\phi\left(A_{0}[i, \cdot]\right)$ and $Z_{1}[i, \cdot]=\phi\left(A_{1}[i, \cdot]\right)$. Otherwise, if $c_{i}=1$ check that $\phi\left(B_{0}[i, \cdot]\right)+Z_{0}[i, \cdot]=0$ and $\phi\left(B_{1}[i, \cdot]\right)+Z_{1}[i, \cdot]=0$. If all the checks pass, the receiver proceeds to the next phase. 
3. Shares Consistency Check Phase For each $b \in\{0,1\}$, Rec randomly chooses a set $T_{b}$ for which $b_{j}=b$ at $M / 2$ coordinates. For each $j \in T_{b}$, Rec checks that there exists a unique $x_{b}^{i}$ such that $A_{b}[i, j]+B_{b}[i, j]=x_{b}^{j}$ for all $i \in[M]$. If so, $x_{b}^{j}$ is marked as consistent. If all shares obtained in this phase are consistent, Rec proceeds to the reconstruction phase. Else it aborts.

4. Reconstruction Phase For $j \in[2 M] / T_{1-b}$, if there exists a unique $x_{b}^{j}$ such that $A_{b}[i, j]+B_{b}[i, j]=x_{b}^{j}$, Rec marks share $j$ as a consistent column. If $R$ obtains less than $M+1$ consistent columns, it aborts. Otherwise, let $x_{b}^{j_{1}}, \ldots, x_{b}^{j_{M+1}}$ be any set of $M+1$ shares obtained from consistent columns. $\operatorname{Rec}$ computes $x_{b} \leftarrow \operatorname{Reconstruct}\left(x_{b}^{j_{1}}, \ldots, x_{b}^{j_{M+1}}\right)$ and outputs $s_{b}=C_{b} \oplus x_{b}$.

We conclude with the following theorem.

Theorem 6.3. Assume the existence of claw-free trapdoor permutations. Then Protocol 6 is a three-round protocol that securely realizes $\mathcal{F}_{\mathrm{OT}}$ with $1 / p$-security in the presence of aborting senders and with privacy in the presence of aborting senders and receivers.

Proof. We consider each corruption case separately.

Privacy Against Malicious Receiver Assume for contradiction, there exists a malicious receiver Rec*, PPT distinguisher $D$, polynomial $p(\cdot)$ and two pairs of inputs $\left(s_{0}^{0}, s_{1}^{0}, z_{0}\right)$ and $\left(s_{0}^{1}, s_{1}^{1}, z_{1}\right)$ such that with probability at least $\frac{1}{p(n)} \operatorname{overPExec}_{\operatorname{Rec}^{*}}\left(1^{n}\right)=$ $\left(\left(m_{s}^{1}, m_{r}\right), \sigma, r_{\operatorname{Rec}}\right)$, it holds for $b=0$ and $b=1$ that

$$
\begin{aligned}
& \mid \operatorname{Pr}\left[m_{s}^{2} \leftarrow \operatorname{Sen}_{2}\left(\sigma, m_{r},\left(s_{0}^{b}, s_{1}^{b}\right)\right): D\left(1^{n}, z_{b}, r_{\operatorname{Rec}},\left(m_{s}^{1}, m_{s}^{2}\right)\right)=1\right] \\
& \quad-\mid \operatorname{Pr}\left[s_{b}^{*} \leftarrow s_{b}^{b} ; s_{1-b}^{*} \leftarrow\{0,1\}^{\ell(n)} ; m_{s}^{2} \leftarrow \operatorname{Sen}_{2}\left(\sigma, m_{r},\left(s_{0}^{*}, s_{1}^{*}\right):\right.\right. \\
& \quad D\left(1^{n}, z_{b}, r_{\operatorname{Rec}},\left(m_{s}^{1}, m_{s}^{2}\right)\right)=1 \mid \geq \frac{1}{p(n)}
\end{aligned}
$$

Recall that in this protocol we repeat the basic OT in parallel $N=6 M$ times. Among these $6 M$ repetitions, the trapdoors for half of them are revealed in the final step. The remaining $3 M$ are used for implementing the OT. We will focus only on these $3 M$ invocations and for simplicity of exposition identify them with indices 1 through $3 M$. Recall that each of the $3 M$ parallel invocations is an instance of Protocol 5 from above that satisfies the privacy definition according to Definition 2.12.

Proof Overview. We will refer to the $3 M$ parallel repetitions as instantiations of our basic OT protocol and the whole protocol as the global OT protocol. On a high-level, we will rely on the privacy of the basic OT protocol to conclude the privacy of the global OT protocol. Observe that for an adversary to violate the privacy of the global OT protocol, there must exist two sets of inputs $\left(s_{0}^{0}, s_{1}^{0}\right)$ and $\left(s_{0}^{1}, s_{1}^{1}\right)$ for the sender such that in the first set, the distinguisher can observe when $s_{0}^{0}$ is replaced by a uniform bit (call 
these experiments resp., Expt ${ }_{0}$ and $\widetilde{\operatorname{Expt}_{0}}$ ) while in the second set the distinguisher can observe when $s_{1}^{1}$ is replaced by a uniform bit (call these experiments resp., Expt $t_{1}$ and $\widetilde{\operatorname{Expt}}_{1}$ ). The intuition behind the proof is that after the malicious receiver delivers the second message there exists an input associated with each of the $3 M$ basic OT instances such that the receiver can only "learn" the corresponding sender's input in each of these instances. Formalizing this intuition is more challenging.

Our first approach (that will not work) is to consider a sequence of $3 M$ hybrids starting from $H_{0}^{0}=\operatorname{Expt}_{0}$ where in the $i$ th hybrid $H_{i}^{0}$ we try to replace one of the two sender's inputs in the first $i$ basic OT instances by a random value. By the privacy of the basic OT protocol, there must exist some sender's input that we can replace by a random value without the distinguisher noticing (beyond a negligible probability). After replacing one of the two inputs in all the $3 M$ invocations, we arrive at a hybrid $H_{3 M}^{0}$ that is not distinguishable from $\operatorname{Expt}_{0}$ by $D$ and where the receiver learns only one of the two values in each of the $3 M$ instances (as the other one is set to random). Recall that in order to learn $s_{0}^{b}$, the receiver must select as its input $b$ in the last $2 M$ basic OT at least $M+1$ times. Therefore, we can conclude that the receiver can learn at most one of the two global inputs $s_{0}^{0}$ and $s_{0}^{1}$. Let the global input that the receiver learns be $s_{0}^{b}$.

In order to arrive at a contradiction, one must use the fact that $D$ distinguishes $\mathrm{Expt}_{0}$ from $\widetilde{\text { Expt }_{0}}$. Using the fact that Expt ${ }_{0}$ cannot be distinguished from $H_{3 M}^{0}$, we have that $D$ also distinguishes $H_{3 M}^{0}$ from $\widetilde{\operatorname{Expt}}_{0}$. However, if $b=1$, then it is indeed possible for $D$ to distinguish $H_{3 M}^{0}$ from $\widetilde{\operatorname{Expt}}_{0}$ because the receiver could learn the global input corresponding to receiver input 1 and this is different in $H_{3 M}^{0}$ and $\widetilde{\mathrm{Expt}}_{0}$, namely $s_{0}^{1}$ in $H_{3 M}^{0}$ and a random bit in $\widehat{\text { Expt }_{0}}$. Hence this approach and sequence of hybrids do not seem to be useful.

Our next idea would be to consider a sequence of hybrids $H_{0}^{1}, \ldots, H_{3 M}^{1}$ starting from

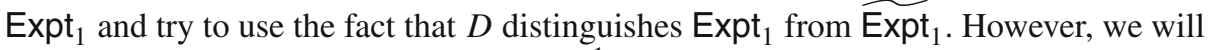
run into the same bottleneck as in hybrid $H_{3 M}^{1}$ it could be the case that the receiver learns the global input $s_{1}^{0}$ and this is what is replaced to a uniform bit in $\widetilde{\operatorname{Expt}_{1}}$.

Our final approach would be to consider four sequences of $3 M$ hybrids simultaneously, a sequence starting from $\operatorname{Expt}_{0},{\widetilde{\operatorname{Expt}_{0}}}_{0}, \operatorname{Expt}_{1}$ and $\widetilde{\operatorname{Expt}}_{1}$, resp., $H_{0}^{0} \cdots H_{3 M}^{0}$, $\widetilde{H}_{0}^{0} \cdots \widetilde{H}_{3 M}^{0}, H_{0}^{1} \cdots H_{3 M}^{1}$ and $\widetilde{H}_{0}^{1} \cdots \widetilde{H}_{3 M}^{1}$. For $b=0$ and $b=1$, Hybrid $H_{i}^{b}$ (resp., $\widetilde{H}_{i}^{b}$ ) will proceed identically to $H_{i-1}^{b}$ (resp., $\widetilde{H}_{i-1}^{b}$ ) with the exception that one of the sender's inputs in the $i$ th basic OT instance is set to a random value. The crucial idea here is that we can pick a single bit $b$ and replace the sender's input corresponding to the same receiver bit $b$ in $H_{i}^{0}, \widetilde{H}_{i}^{0}, H_{i}^{1}$ and $\widetilde{H}_{i}^{1}$. This is because, the privacy definition of the basic OT protocol provides such a guarantee, namely, after the second message, for any pair of inputs for the sender there exists only one input bit corresponding to which any distinguisher can notice when replaced by a random value. Now, based on which sender's input is replaced by a random value in each of the last $2 M$ basic OT protocols, we know which of the global inputs the receiver will learn. If it learns the global input corresponding to receiver bit $b$, then we can show that $H_{3 M}^{1-b}$ and $\widetilde{H}_{3 M}^{1-b}$ are identically distributed. This is because the difference between $H_{3 M}^{1-b}$ and $\widetilde{H}_{3 M}^{1-b}$ is in the sender's global input corresponding to receiver bit $1-b$ and corresponding to this sender input, 
where at most $M-1$ shares have not been replaced by a random value in both $H_{3 M}^{1-b}$ and $\widetilde{H}_{3 M}^{1-b}$. Now, since $M-1$ shares statistically hide the global sender's input corresponding to receiver bit $1-b$, the distributions for $H_{3 M}^{1-b}$ and $\widetilde{H}_{3 M}^{1-b}$ must be identical. However, we know that the probability with which $D$ distinguishes $H_{3 M}^{1-b}$ from Expt fro $_{1-b}$ and $\widetilde{H}_{3 M}^{1-b}$ from $\widetilde{\operatorname{Expt}}_{1-b}$ is small but $D$ distinguishes Expt ${ }_{1-b}$ from $_{\text {Expt }_{1-b}}$ with large probability, we arrive at a contradiction. This concludes the proof overview and we now proceed to prove it formally.

For each $b \in\{0,1\}$, we will consider two sequences of hybrids $H_{0}^{b}, H_{1}^{b}, \ldots, H_{3 M}^{b}$ and $\widetilde{H}_{0}^{b}, \widetilde{H}_{1}^{b}, \ldots, \widetilde{H}_{3 M}^{b}$ where $H_{0}^{b}$ is the hybrid where the sender's message is generated according to the honest strategy with inputs $\left(s_{0}^{b}, s_{1}^{b}\right)$ and $\widetilde{H}_{0}^{b}$ will be identical to $H_{0}^{b}$ with the exception that $s_{1-b}^{0}$ is replaced to random. We will successively define the next hybrids as follows: Hybrid $H_{i}^{b}$ (resp., $\widetilde{H}_{i}^{b}$ ) will proceed identically to $H_{i-1}^{b}$ (resp., $\widetilde{H}_{i-1}^{b}$ ) with the exception that we will replace one of the two OT inputs of the sender in the $i$ th OT invocation with a random bit. We will chose that input for which the probability with which $D$ can distinguish the change is at most $\frac{1}{4 N p(n)}$. There must exist one such input since otherwise the $i$ th OT will violate the privacy of that individual OT execution. Furthermore, we can assume that there exists a single bit $b^{*}$ such that where we replace the sender's input corresponding to $b^{*}$ from $H_{i-1}^{0}$ to $H_{i}^{0}$, from $H_{i-1}^{1}$ to $H_{i}^{1}$ and the analogous hybrids in the $\widetilde{H}^{0}$ and $\widetilde{H}^{1}$ sequence, the distinguisher can distinguish with probability at most $\frac{1}{4 N p(n)}$. This is because otherwise we can construct an adversary that violates the privacy of the $i$ th OT instance used in our protocol. ${ }^{9}$ If the distinguishing probability for both inputs is small, we pick one of them arbitrarily and switch to random in this hybrid.

Finally, we can identify in $H_{3 M}^{b}$ which of the sender's inputs $s_{0}^{b}$ and $s_{1}^{b}$ in the overall protocol can the receiver "possibly learn." This is because we secret share the input in a specific way where the first $M$ OTs do not reveal anything about either of the two inputs and are used only for consistency checks and for the next $2 M$ executions the receiver needs to obtain at least $M+1$ shares of the same input to reconstruct the secret. By looking at which of the inputs the receiver obtains in the $2 M$ executions, we find that one that occurs at least $M+1$ times. Furthermore, the input it learns $H_{3 M}^{0}, H_{3 M}^{1}, \widetilde{H}_{3 M}^{0}, \widetilde{H}_{3 M}^{1}$ must all correspond to the same receiver bit. Now, suppose that this is the bit 0 . This means that the distribution of the inputs to the individual OT invocations must be identical in $H_{3 M}^{0}$ and $\widetilde{H}_{3 M}^{0}$. This is because the only difference in the inputs chosen from $H_{3 M}^{0}$ and $\widetilde{H}_{3 M}^{0}$ is that input of the sender corresponding to receiver bit 1 is $s_{1}^{1}$ in the $H_{3 M}^{0}$ and is sampled randomly in $\widetilde{H}_{3 M}^{0}$ and then secret shared. Since the receiver does not receive sufficiently many shares the input is statistically hidden. In other words, the set of shares that are revealed corresponding to receiver bit 1 in these two hybrids are distributed identically. Now, to arrive at contradiction we observe that $H_{0}^{0}$ is at most $N \times \frac{1}{4 N p(n)}=\frac{1}{4 p(n)}$ far from $H_{3 M}^{0}$ and $\widetilde{H}_{0}^{0}$ is at most $\frac{1}{4 p(n)}$ far from $\widetilde{H}_{3 M}^{0}$. This means that $H_{0}^{0}$ and $\widetilde{H}_{0}^{0}$ are at most $\frac{1}{2 p(n)}$ far. But this is a contradiction to Eq. 2 for $b=0$.

\footnotetext{
${ }^{9}$ In this reduction, we will consider an adversary $\mathcal{A}^{*}$ and distinguisher $\mathcal{D}^{*} . \mathcal{A}^{*}$ will incorporate $\operatorname{Rec}{ }^{*}$ and simulates everything internally except the forward the messages in the $i$ th instance externally (the auxiliary input will provide the inputs for the sender in all the other OT instances that are not forwarded).
} 
$1 / p$-simulation Against Malicious Sender Recall that when the sender is corrupted we need to prove $1 / p$-indistinguishability. More precisely, we need to define a simulator that produces a view of the malicious sender $\mathcal{A}$ while extracting both $s_{0}$ and $s_{1}$, where the view and the value learned by the honest receiver is $1 / p$-indistinguishable from the sender's real view and the receiver's output in a real execution. More precisely, for any probabilistic polynomial-time adversary $\mathcal{A}$ controlling Sen we define a simulator $\mathcal{S}$ that proceeds as follows:

1. $\mathcal{S}$ invokes $\mathcal{A}$ on its input and randomness of appropriate length.

2. Upon receiving from $\mathcal{A}$ the first message, $\mathcal{S}$ computes the second message honestly with input $b=0$. Let $\widetilde{\operatorname{Trap}}$ contain the indices for which it requests the trapdoor and $[N]-\widetilde{\operatorname{Trap}}=\left\{a_{1}, \ldots, a_{3 M}\right\}$. If $\mathcal{A}$ aborts before sending the third message then $\mathcal{S}$ outputs the view of $\mathcal{A}$ and halts.

3. Otherwise, upon receiving $\mathcal{A}$ 's third message, $\mathcal{S}$ records the set $\left\{\mathrm{tk}_{j}^{0}\right\}_{j \in \text { Trap }}$. Next, it stalls the main execution temporarily and proceeds to rewind $\mathcal{A}$. Specifically, $\mathcal{S}$ rewinds $\mathcal{A}$ to the second message and proceeds as follows:

- For every $i \in[3 M], \mathcal{S}$ rewinds $\mathcal{A}$ for $T=N^{4} p$ attempts, where in each such attempt $\mathcal{S}$ supplies a uniformly random second message according to the receiver's strategy with input $b=0$, conditioned on $a_{i} \in$ Trap. In each rewinding, $\mathcal{S}$ collects the trapdoor for index $a_{i}$, i.e., $\mathrm{tk}_{a_{i}}^{0}$.

4. If upon concluding the rewinding phase $\mathcal{S}$ does not obtain the trapdoors for at least $1-1 / 3 p$ fraction of $i \in[N]-\widetilde{\text { Trap }}$, then it halts outputting fail.

5. Otherwise, let $I \subseteq\left\{a_{1}, \ldots, a_{M}\right\}$ and $J \subseteq\left\{a_{M+1}, \ldots, a_{3 M}\right\}$ be the sets of indices for which it has a trapdoor. Next, it returns to the main execution and tries to extract both the sender's OT inputs as follows. First, it performs the share consistency check and the share validity check as the honest receiver would and if either of them fail, then the simulator halts outputting the view of $\mathcal{A}$.

6. Otherwise, for every $j \in J$, since it has the trapdoor, it will be able to extract both the sender's inputs for these smaller OT instances. For each $b \in\{0,1\}$ and $j \in J$, if there exists a unique $x_{b}^{j}$ such that $A_{b}[i, j]+A_{b}[i, j]=x_{b}^{j}, \mathcal{S}$ marks column $j$ as consistent. If it obtains at least $M+1$ shares for $x_{b}$ from consistent columns it reconstructs $x_{b}$, and then obtains $s_{b}$ from $x_{b}$ and $C_{b}$. If not, it sets $s_{b}=\perp$.

7. Finally, $\mathcal{S}$ forwards $\left(s_{0}, s_{1}\right)$ to $\mathcal{F}_{\text {Oт }}$ and halts, outputting whatever $\mathcal{A}$ does.

Clearly, $\mathcal{S}$ runs in strict polynomial-time. We next prove the correctness of simulation. On a high-level, the second message in all the rewinding attempts is generated identically to the second message of the real execution and is independent of the bit $b$ that the receiver enters. This follows because we repeat the basic protocol in parallel and in each of these instances the receiver's message statistically hides its input. Let $s=\log ^{2} n$, then two cases arise:

1. The Abort Probability of the Sender is Higher than $1-\frac{1}{N p}$ In this case, $1 / p$ indistinguishability is achieved directly as the simulation outputs views on which the sender aborts with at least the same probability as such views occur in the real view. Now, since this accounts for a probability mass of at least $1-\frac{1}{N p}>1-\frac{1}{p}$, $1 / p$-indistinguishability follows. 
2. The Abort Probability of the Sender is at most $1-\frac{1}{N p}$. In this case by setting $M>s p$, we argue that except with negligible probability (roughly, $2^{-O(s)}$ ), the simulator will be able to obtain the trapdoors, for at least $1-\frac{1}{3 p}$ fraction of the indices in $\left\{a_{1}, \ldots, a_{3 M}\right\}$ via rewinding. This is formally proven in Claim 5.1. Just as in the previous protocol, we have that for every index in $J$ that the simulator obtains a trapdoor, it will be able to extract both of the sender's inputs. Specifically, as $M>s p$, we can conclude that the simulator fails to obtain the trapdoor of at most $\frac{1}{3 p} \times 3 M=s$ indices. This means that $|J| \geq 2 M-s$ indices.

Next, from the shares consistency check we can conclude that with very high probability all but $s$ columns contain shares that are consistent. From the shares validity check, we can conclude that with very high probability there is a single row $i_{b}$ corresponding to each $b \in\{0,1\}$ such that $A_{b}[i, \cdot]+B_{b}[i, \cdot]$ contains valid shares of some secret. Combining these checks, we can conclude that there are at least $2 M-s$ columns that are consistent, i.e., the shared value in each row is the same and therefore must equal $A_{b}\left[i_{b}, \cdot\right]+B_{b}\left[i_{b}, \cdot\right]$. Furthermore, from the statistically binding property of the commitment scheme we can conclude that for any one of these consistent columns, there can be only one value for the shares that can be extracted by both the receiver and the simulator.

In this case, we can now conclude that, using the trapdoors, the simulator obtains at least $2 M-s-s$ shares for both inputs among the consistent columns. Since $M>s p$ we have that $2 M-2 s>M+1$ (for $p>2$ ) and from $M+1$ valid shares it can extract $s_{b}$ for each $b \in\{0,1\}$.

To conclude, we prove the following corollary regarding input-indistinguishable computation in Appendix B.

Corollary 6.4. Protocol 6 satisfies Definition A.5, namely, input-indistinguishability [51].

It is conceivable that our OT protocol when combined with the two-round [43] protocol will yield a 3-round secure computation protocol that satisfies input-indistinguishability and leave it as future work.

\section{On the Impossibility of Black-Box 3-Round $2 \mathrm{PC}$ with $1 / \mathrm{p}-$ Security}

In this section, using ideas from the 3-round lower bound of Goldreich and Krawczyk [28], we show that achieving $1 / p$-security against receivers is impossible.

First, we define a notion of robustness analogous to one presented in [44] in the context of multiparty computation. Robustness is a weaker requirement than correctness and, informally, requires that no honest party outputs a value not in the range of the function. For simplicity, we define robustness only for boolean functions.

Definition 7.1. We say that a two-party secure computation $\left\langle P_{1}, P_{2}\right\rangle$ protocol computing a function $f:\{0,1\}^{*} \times\{0,1\}^{*} \rightarrow\{0,1\}$ is robust against a malicious $P_{1}$ if every PPT adversary $\mathcal{A}$ controlling party $P_{1}$ in an interaction with $P_{2}$ cannot make $P_{2}$ 
on input $y$ output $b$ with more than negligible probability, if there exists no $x$ such that $f(x, y)=b$.

In this section, we prove two lower bounds:

1. Assuming NP $\nsubseteq$ BPP, there exists no 3-round black-box construction of a secure two-party computation protocol that is robust against malicious senders and achieves $1 / p$-security against malicious receivers.

2. There exists no 3-round black-box construction of an oblivious-transfer protocol that achieves privacy against malicious senders and $1 / p$-security against malicious receivers.

The first result shows that constructing correct protocols with $1 / p$-simulation of receivers is impossible using black-box techniques and will essentially follow using a generalization of the [28] result. The second result shows that achieving $1 / p$-security against malicious receivers is impossible if we want privacy against malicious senders. In essence, this proves that our protocol from Sect. 6 is tight if we require privacy against both parties.

More formally, our first lower bound result is the following.

Theorem 7.2. Unless $N P \subseteq B P P$, there exists no three rounds black-box construction of a secure two-party protocol with $1 /$ p-security in the presence of aborting receivers and with privacy in the presence of aborting senders that realizes arbitrary polynomialtime computable functionalities.

Proof. We rely on the following lemma, that follows from the 3-round lower bound for zero-knowledge (ZK) interactive proofs of Goldreich and Krawczyk [28].

Lemma 7.3. Unless $N P \subseteq B P P$, there exists no black-box three-round zero-knowledge interactive proofs for all of NP with $1 /$ p-security.

Given the proof of Lemma 7.3, the theorem follows as a corollary. Consider an arbitrary NP-language $L$ with witness relation $R_{L}$. Then, for any $x \in\{0,1\}^{*}$ consider the functionality $f_{x}:\{0,1\}^{*} \rightarrow\{0,1\}$ that on input $w$ from $P_{1}$ outputs $R_{L}(x, w)$ to party $P_{2}$. In essence, a secure computation protocol for this functionality yields a zeroknowledge interactive proof. Moreover, it follows from the simulation-based definition of the $1 / p$-security that if the original secure protocol is only $1 / p$-secure, we get a zero-knowledge proof that is $1 / p$-secure.

We now provide a brief overview of why Lemma 7.3 holds. We first recall the lower bound of Goldreich and Krawczyk. Suppose that there exists a 3-round ZK proof for an arbitrary NP language $L$. Consider a pseudo-random function family $F=\left\{f_{n}\right\}_{n \in\{0,1\}^{*}} .^{10}$ Then define a malicious verifier $V_{n}^{*}$ that incorporates a function $f_{n}$ from the PRF family $F$, and generates its second message of the $\mathrm{ZK}$ protocol by first generating randomness $\tau$ by applying $f_{n}$ to the prover's first message and the running the honest verifier's

\footnotetext{
${ }^{10}$ For simplicity, we present the proof with PRF's. However, to get an unconditional result as stated in the lemma, we can rely on $m$-wise independent hash-function family where $m$ is polynomially related to the expected running time of the simulator $\mathcal{S}$.
} 
code $\mathcal{V}$ with random tape set to $\tau$. Consider the simulator $\mathcal{S}$ that simulates this family of malicious verifiers $\mathcal{V}_{n}^{*}$. The main idea here is that using the simulation $\mathcal{S}$ and $\mathcal{V}_{n}^{*}$ we can show that either $L \in$ BPP or the interactive proof is not sound (which is the analogue notion to robustness in zero-knowledge protocols). On a high level, from the pseudorandomness of the family $F$ it follows that the real view generated by $\mathcal{V}_{n}^{*}$ is indistinguishable from the view that is generated by the real verifier $\mathcal{V}$. Hence, given input $x \in L, \mathcal{S}^{\mathcal{V}_{n}^{*}}$ produces a convincing view for the verifier with probability $q$ that is negligibly close to 1 . Moreover, on input $x \notin L, \mathcal{S} \mathcal{V}_{n}^{*}$ either produces a convincing view or not. Concretely,

- If it does not produce a view with probability close to $q$ for any $x \notin L$, then we can use $\mathcal{S}^{\mathcal{V}_{n}^{*}}$ as a BPP-decider for the language $L$ by simply estimating the probability with which $\mathcal{S}^{*}(x)$ outputs a convincing view.

- If it does produce a view with some probability close to $q$ for some $x \notin L$, then we can construct a malicious prover $\mathcal{P}^{*}$ that convinces the honest verifier $\mathcal{V}$ of the statement $x$ with non-negligible probability, which contradicts the soundness of the interactive proof. First, we observe that the view output by $\mathcal{S}^{\mathcal{V}_{n}^{*}}$ is indistinguishable from the output of $\mathcal{S}^{\tilde{\mathcal{V}}}$ where $\tilde{\mathcal{V}}$ uses a truly random function instead of a PRF function $f_{n}$ to generate the randomness. Specifically, given input $x, \mathcal{P}^{*}$ internally simulates $\mathcal{S}^{\widetilde{\mathcal{V}}}(x)$ by emulating the random function queries. ${ }^{11}$ It then randomly chooses a session from the internal emulation and forwards the messages exchanged between $\mathcal{S}$ and $\tilde{\mathcal{V}}$ to the external honest verifier. It follows that the view generated internally by $\mathcal{P}$ is identically distributed to the view generated by $\mathcal{S}^{\tilde{\mathcal{V}}}(x)$. Furthermore, if the view output by $\mathcal{S}$ is the session forwarded externally to the honest verifier, then it implies that the external verifier essentially accepts. ${ }^{12} \mathrm{Fi}$ nally, suppose that the simulation runs in time $T$, then it follows that $\mathcal{P}^{*}$ guesses the correct session to forward outside with probability $\frac{q}{T}$. Therefore, it convinces the external verifier with probability close to $\frac{q}{T}$. Now, since $T$ is some polynomial, it follows that $\mathcal{P}^{*}$ convinces $\mathcal{V}$ on an input $x \notin L$ with non-negligible probability and this violates soundness.

We now conclude the proof of the lemma by making the observation that even if the simulation was only $1 / p$-indistinguishable, then $q=1-\frac{1}{p}$ and the success probability of $\mathcal{P}^{*}$ is still non-negligible.

For the second lower bound result we prove that:

Theorem 7.4. For $\frac{1}{p}<\frac{1}{2}-\frac{1}{\text { poly }(n)}$, there exists no three rounds oblivious-transfer protocol that achieves privacy in the presence of aborting senders and $1 /$ p-(black-box) security in the presence of aborting receivers.

\footnotetext{
${ }^{11}$ Namely, on any input query to the random function, $\mathcal{P}^{*}$ checks if the query has already been asked and produces the same answer in this case. Otherwise, it samples and feeds a uniform output and records the query/answer pair.

${ }^{12}$ This is not entirely accurate as $\mathcal{P}^{*}$ does not know the actual randomness used by the external verifier since this is a private-coin protocol. Nevertheless, it is possible to formally prove that conditioned on $\mathcal{P}^{*}$ guessing correctly, $\mathcal{P}^{*}$ convinces the external verifier with probability equal to the probability $\mathcal{S}$ outputs a convincing view in the internal emulation, i.e., close to $q$.
} 
Proof Sketch: We follow a similar approach as in our previous construction. Suppose we have a 3-round oblivious-transfer protocol that achieves $1 / p$-simulation against malicious receivers. We show that such a protocol cannot be private against malicious senders. More formally, we can construct a malicious sender Sen* and distinguisher $D$ that can distinguish the sender's view when the receiver's input is 0 and 1 with non-negligible probability and this violates privacy against malicious senders.

From the $1 / p$-simulation property, we know there exists a black-box simulation $\mathcal{S}$ that can simulate arbitrary malicious receivers. Analogous to [28], we construct a malicious receiver $\operatorname{Rec}_{b}$ that on input $b$, samples its random tape by applying a PRF to the sender's first message and completes the execution. It is now guaranteed that $\mathcal{S}$ can simulate $\operatorname{Rec}_{b}$ and will extract the value $b$, and upon receiving $s_{b}$ from the ideal functionality produces a view of $\operatorname{Rec}_{b}$ with $1 / p$ indistinguishability. As in the previous proof, we can emulate a modified version of $\operatorname{Rec}_{b}$, denoted by $\operatorname{Rec}_{b}^{*}$, that does not use a PRF to sample the random tape for every session but simply picks a fresh random tape for every session the simulation starts.

Assume that $\mathcal{S}$ runs in $T$ time. ${ }^{13}$ This means that $\mathcal{S}$ can open at most $T$ sessions with $\operatorname{Rec}_{b}^{*}$. We now consider a sequence of hybrid experiments where we emulate a malicious receiver to $\mathcal{S}$ starting from $\operatorname{Rec}_{0}^{*}$ and ending in $\operatorname{Rec}_{1}^{*}$ : In hybrid experiment $H_{i}$ : we emulate the receiver's message according to $\operatorname{Rec}_{1}^{*}$ 's strategy in the first $i$ sessions and $\operatorname{Rec}_{0}^{*}$ in the remaining sessions. Observe that $H_{0}$ is identical to the game with $\operatorname{Rec}_{0}^{*}$ and $H_{T}$ is the same as the game with $\operatorname{Rec}_{1}^{*}$. Furthermore, we know that the games with $\operatorname{Rec}_{0}^{*}$ and $\operatorname{Rec}_{1}^{*}$ must be distinguishable with probability at least $1-2 / p$ since the simulator sends 0 to the ideal functionality with $\operatorname{Rec}_{0}^{*}$ with probability at least $1-1 / p$ and 1 with $\operatorname{Rec}_{1}^{*}$ with probability $1-1 / p$. This means that there exists an $i$ such that the experiments $H_{i-1}$ and $H_{i}$ can be distinguished with probability at least $\frac{1}{T}(1-2 / p)$ which is non-negligible since $\frac{1}{p}<1 / 2-1 /$ poly and $T$ is polynomial. Notice that the only difference between hybrids $H_{i-1}$ and $H_{i}$ is in the distribution of the receiver's message in the $i$ th session. This means that we can now use $i$, experiments $H_{i-1}$ and $H_{i}$ to distinguish the receiver's message when its input is 0 and input 1 by constructing a malicious sender that emulates the experiment $H_{i}$ internally and feeds the message received from outside internally in the $i$ th session. This violates the privacy requirement against malicious senders.

\section{A Preliminaries - Appendix}

\section{A.1 Public Key Encryption Schemes (PKE)}

We specify the definitions of public key encryption and IND-CPA.

Definition A.1. $(P K E)$ We say that $\Pi=$ (Gen, Enc, Dec) is a public key encryption scheme if Gen, Enc, Dec are polynomial-time algorithms specified as follows:

\footnotetext{
${ }^{13}$ It is possible to extend this argument to expected polynomial-time simulators by using a Markov argument.
} 
- Gen, given a security parameter $n$ (in unary), outputs keys (PK, SK), where PK is a public key and SK is a secret key. We denote this by (PK, SK) $\leftarrow$ Gen $\left(1^{n}\right)$.

- Enc, given the public key PK and a plaintext message $m$, outputs a ciphertext $c$ encrypting $m$. We denote this by $c \leftarrow \operatorname{Enc}_{\mathrm{PK}}(m)$; and when emphasizing the randomness $r$ used for encryption, we denote this by $c \leftarrow \operatorname{Enc}_{\mathrm{PK}}(m ; r)$.

- Dec, given the public key PK, secret key SK and a ciphertext $c$, outputs a plaintext message $m$ s.t. there exists randomness $r$ for which $c=\operatorname{Enc}_{\mathrm{PK}}(m ; r)$ (or $\perp$ if no such message exists). We denote this by $m \leftarrow \operatorname{Dec}_{\mathrm{PK}, \mathrm{SK}}(c)$.

For a public key encryption scheme $\Pi=($ Gen, Enc, Dec) and a PPT adversary $\mathcal{A}=$ $\left(\mathcal{A}_{1}, \mathcal{A}_{2}\right)$, we consider the following IND-CPA game denoted by $\operatorname{ADV}_{\Pi, \mathcal{A}}(n)$ :

$$
\begin{aligned}
& (\mathrm{PK}, \mathrm{SK}) \leftarrow \operatorname{Gen}\left(1^{n}\right) . \\
& \left(m_{0}, m_{1}, \text { history }\right) \leftarrow \mathcal{A}_{1}(\mathrm{PK}), \text { s.t. }\left|m_{0}\right|=\left|m_{1}\right| . \\
& c \leftarrow \operatorname{Enc}_{\mathrm{PK}}\left(m_{b}\right), \text { where } b \leftarrow R\{0,1\} . \\
& b^{\prime} \leftarrow \mathcal{A}_{2}(c, \text { history }) .
\end{aligned}
$$

Return 1 if $b^{\prime}=b$, and 0 otherwise.

Definition A.2. (IND-CPA) A public key encryption scheme $\Pi=$ (Gen, Enc, Dec) has indistinguishable encryptions under chosen plaintext attacks (IND-CPA), if for every PPT adversary $\mathcal{A}=\left(\mathcal{A}_{1}, \mathcal{A}_{2}\right)$ there exists a negligible function negl such that

$$
\operatorname{Pr}\left[\operatorname{ADv}_{\Pi, \mathcal{A}}(n)=1\right] \leq \frac{1}{2}+\operatorname{negl}(n)
$$

where the probability is taken over the random coins used by $\mathcal{A}$, as well as the random coins used in the experiment.

\section{A.1.1 The El Gamal PKE}

A useful implementation of homomorphic PKE is the El Gamal [27] scheme that is multiplicatively homomorphic. In this paper, we exploit the additive variation. Let $\mathbb{G}$ be a group of prime order $p$ in which DDH is hard. Then the public key is a tuple $\mathrm{PK}=\langle\mathbb{G}, p, g, h\rangle$ and the corresponding secret key is $\mathrm{SK}=s$, s.t. $g^{s}=h$. Encryption is performed by choosing $r \leftarrow \mathbb{Z}_{p}$ and computing Enc $\mathrm{EKK}(m ; r)=\left\langle g^{r}, h^{r} \cdot m\right\rangle$. Decryption of a ciphertext $C=\langle\alpha, \beta\rangle$ is performed by computing $m=\beta \cdot \alpha^{-s}$ and then finding $m$ by running an exhaustive search.

\section{A.2 Knowledge Extraction}

In this paper, we are interested in witness-indistinguishable and zero-knowledge proofs that are proofs of knowledge (PoK) which imply the existence of a knowledge extractor that extracts the witness $w$ used by the prover. 
Definition A.3. Let $R$ be a binary relation and $\kappa \rightarrow[0,1]$. We say that an interactive function $\mathcal{V}$ is a knowledge verifier for the language $L$ with knowledge error $\kappa$ if the following two conditions hold:

Non-triviality There exists an interactive machine $\mathcal{P}$ such that for every $(x, w)$ such that $w$ is a witness for $x \in L$, all possible interactions of $\mathcal{V}$ with $\mathcal{P}$ on common input $x$ and auxiliary input $w$ are accepting.

Validity (with error $\kappa$ ) There exists a polynomial $q(\cdot)$ and a probabilistic oracle machine $K$ such that for every interactive function $\mathcal{P}$, every $x \in L$, and every machine $K$ satisfies the following condition:

Denote by $p(x, y, r)$ the probability that the interactive machine $\mathcal{V}$ accepts, on input $x$, when interacting with the prover specified by $\mathcal{P}_{x, y, r}$ that uses randomness $r$ (where the probability is taken over the coins of $\mathcal{V}$ ). If $p(x, y, r)>\kappa(|x|)$, then, on input $x$ and with access to oracle $\mathcal{P}_{x, y, r}$, machine $K$ outputs a witness $s$ for $x \in L$ within an expected number of steps bounded by

$$
\frac{q(|x|)}{p(x, y, r)-\kappa(|x|)}
$$

The oracle machine $K$ is called a universal knowledge extractor.

It is known that any $\Sigma$-protocol is a WI-PoK. One such example is the protocol for proving the knowledge of Hamiltonian cycle in a graph, which is an NP-complete problem.

\section{A.3 Input-Indistinguishable Computation [51]}

Below, we recall the definition of input-indistinguishable computation of [51] which provided a definition in the concurrent setting where the parties interact in $m$ concurrent interactions. We present the definition as is from [51]; however, we will rely on the definition for the case $m=1$. We specify some notations first. For an integer $n$, we let $\operatorname{EXEC}_{P_{1}, P_{2}}\left(\mathbf{x}, \mathbf{y}, 1^{n}\right)$ denote the random variable obtained by randomly and independently selecting random tapes for the parties and executing the protocol $\pi$ between $P_{1}$ and $P_{2}$ on respective inputs $\mathbf{x}$ and $\mathbf{y}$ and random tapes $\rho_{1}, \rho_{2}$. Let $e$ be an execution that consists of $m$ concurrent sessions of $\pi$. For a positive integer $i \in[m]$, let $M_{1}^{i}$ be the sequence of messages received by the first party in session $i$. The first-party view of session $i$ in $e$, denoted $\operatorname{View}_{1}^{i}(e)$, is defined to be $\left(x_{i}, \rho_{1}^{i}, M_{1}^{i}\right)$. Symmetrically defined is the second-party view of session $i, \operatorname{View}_{2}^{i}(e)$. For $(i, j) \in[m] \times[k]$, define a Boolean variable $\operatorname{ABORT}(i, j)(e)$ to be true if and only if session $i$ in $e$ is aborted by round $j$. Finally, define a Boolean variable $\operatorname{OUTPUT}_{1}^{i}(e)$ to be true if and only if the output delivery message has been sent to party $P_{1}$ in session $i$ in $e$. $\operatorname{OUTPUT}_{2}^{i}(e)$ is symmetrically defined.

Definition A.4. (Implicit input) Let $\pi$ be a $k$-round protocol, and let $P_{1}^{*}$ be an $m$ concurrent adversary. Consider a function, $\mathrm{IN}_{1}$, that maps the full view, view $w_{1}^{*}(e)$, in an execution $e$ of $\left(P_{1}^{*}, P_{2}\right)$, into a sequence $\mathbf{x}^{*}=\left(x_{1}^{*}, \ldots, x_{m}^{*}\right) \in\left(\mathcal{D}_{1} \cup \perp\right)^{m}$. The function 
is said to be a first party implicit input function function for $\pi$ if for any $i \in[m]$ for which $\operatorname{ABORT}^{(i, k-1)}(e)$ is true, the value $x_{i}^{*}$ equals $\perp$. The notion of a second-party implicit input, $\mathrm{IN}_{2}$, is symmetrically defined.

Definition A.5. (Input-indistinguishable computation) Let $f: \mathcal{D}_{1} \times \mathcal{D}_{2} \mapsto \mathcal{R}_{1} \times \mathcal{R}_{2}$ be a deterministic function, and let $\pi$ be a fixed-round two-party protocol. We say that $\pi$ securely computes $f$ with respect to the first party and implicit input function $\mathrm{IN}_{2}$, if for every polynomial $m=m(n)$, the following conditions hold:

1. Completeness For every $(\mathbf{x}, \mathbf{y}) \in\left(\mathcal{D}_{1}\right)^{m} \times\left(\mathcal{D}_{2}\right)^{m}$, every $n \in \mathbb{N}$, and every $i \in[m]$ :

$$
\operatorname{Pr}\left[\operatorname{View}_{P_{1}}(e)=f_{1}\left(x_{i}, y_{i}\right)\right]=1
$$

where $e \leftarrow \mathrm{EXEC}_{P_{1}, P_{2}}\left(\mathbf{x}, \mathbf{y}, 1^{n}\right)$.

2. Implicit Computation For every efficient m-concurrent ITM $P_{2}^{*}$, there exists a negligible function $\mu: \mathbb{N} \mapsto \mathbb{N}$, so that for every $(\mathbf{x}, \mathbf{y}) \in \mathcal{D}_{1}^{m} \times \mathcal{D}_{2}^{2}$, every $n \in \mathbb{N}$, and every $i \in[m]$ :

$$
\operatorname{Pr}\left[P_{1}\left(\mathbf{V i e w}_{1}^{i}(e)\right)=\left\{\begin{array}{c}
f_{1}\left(x_{i}, y_{i}^{*}\right) \operatorname{OUTPUT}_{1}^{i}(e) \\
\perp \neg \operatorname{OUTPUT}_{1}^{i}(e)
\end{array}\right\}\right] \geq 1-\mu(n)
$$

where $e \leftarrow \operatorname{EXEC}_{P_{1}, P_{2}^{*}}\left(\mathbf{x}, \mathbf{y}, 1^{n}\right), y^{*} \leftarrow \operatorname{IN}_{2}\left(\operatorname{View}_{2}^{*}(e)\right)$.

3. Input Indistinguishability and Independence For every efficient $m$-concurrent ITM $P_{2}^{*}$, every $\mathbf{x}^{1}, \mathbf{x}^{2} \in \mathcal{D}_{1}^{m}$, and every $\mathbf{y} \in \mathcal{D}_{2}^{m}$, the following ensembles are computationally indistinguishable:

- $\left\{\operatorname{Expt}_{P_{1}, P_{2}^{*}}\left(\mathbf{x}^{1}, \mathbf{x}^{2}, \mathbf{y}, 1^{n}\right)\right\}_{n \in \mathbb{N}}$

- $\left\{\operatorname{Expt}_{P_{1}, P_{2}^{*}}\left(\mathbf{x}^{2}, \mathbf{x}^{1}, \mathbf{y}, 1^{n}\right)\right\}_{n \in \mathbb{N}}$

where the random variable $\operatorname{Expt}_{P_{1}, P_{2}^{*}}\left(\mathbf{x}^{1}, \mathbf{x}^{2}, \mathbf{y}, 1^{n}\right)$ is defined as follows:

1. $e \leftarrow \operatorname{EXEC}_{P_{1}, P_{2}^{*}}\left(\mathbf{x}^{1}, \mathbf{y}, 1^{n}\right)$

2. $\mathbf{y}^{*} \leftarrow \mathrm{IN}_{2}\left(\operatorname{View}_{2}^{*}(e)\right)$

3. If there exists $i \in[m]$ for which $\operatorname{OUTPUT}_{i}^{2}(e)$ is true, and $f_{2}\left(x_{1}^{i}, y_{i}^{*}\right) \neq$ $f_{2}\left(x_{2}^{i}, y_{i}^{*}\right)$ then output $\perp$

4. Otherwise, output $\left(\mathbf{y}^{*}, \mathbf{V i e w}_{2}^{*}(e)\right)$

Secure computation with respect to the second party is symmetrically defined. We finally say that $\pi$ securely computes $f$, if there exist implicit input functions $I_{1}, I_{1}$ such that $\pi$ securely computes $\mathrm{f}$ with respect to both the first and the second party, and $\mathrm{IN}_{1}, \mathrm{IN}_{2}$.

\section{A.4 Secure Two-Party Computation}

We briefly present the standard definition for secure multiparty computation and refer to [35, Chapter 7] for more details and motivating discussions. A two-party protocol problem is cast by specifying a random process that maps pairs of inputs to pairs of outputs (one for each party). We refer to such a process as a functionality and denote it $f:\{0,1\}^{*} \times\{0,1\}^{*} \rightarrow\{0,1\}^{*} \times\{0,1\}^{*}$, where $f=\left(f_{1}, f_{2}\right)$. That is, for every pair of 
inputs $(x, y)$, the output vector is a random variable $\left(f_{1}(x, y), f_{2}(x, y)\right)$ ranging over pairs of strings where $P_{1}$ receives $f_{1}(x, y)$ and $P_{2}$ receives $f_{2}(x, y)$. We use the notation $(x, y) \mapsto\left(f_{1}(x, y), f_{2}(x, y)\right)$ to describe a functionality. We prove the security of our protocols in the settings of malicious computationally bounded adversaries. Security is analyzed by comparing what an adversary can do in a real protocol execution to what it can do in an ideal scenario. In the ideal scenario, the computation involves an incorruptible trusted third party to whom the parties send their inputs. The trusted party computes the functionality on the inputs and returns to each party its respective output. Informally, the protocol is secure if any adversary interacting in the real protocol (i.e., where no trusted third party exists) can do no more harm than what it could do in the ideal scenario. In this paper, we follow the $\frac{1}{p}$-secure computation definition from [29] which presented a simulation based definition for which the difference between the real and the simulated distributions differ within $\frac{1}{p}$.

Execution in the Ideal Model In an ideal execution, the parties submit inputs to a trusted party, that computes the output. An honest party receives its input for the computation and just directs it to the trusted party, whereas a corrupted party can replace its input with any other value of the same length. Since we do not consider fairness, the trusted party first sends the outputs of the corrupted parties to the adversary, and the adversary then decides whether the honest parties would receive their outputs from the trusted party or an abort symbol $\perp$. Let $f$ be a two-party functionality where $f=\left(f_{1}, f_{2}\right)$, let $\mathcal{A}$ be a PPT algorithm, and let $I \subset$ [2] be the set of corrupted parties (either $P_{1}$ is corrupted or $P_{2}$ is corrupted or neither). Then, the ideal execution of $f$ on inputs $(x, y)$, auxiliary input $z$ to $\mathcal{A}$ and security parameter $n$, denoted $\operatorname{IDEAL}_{f, \mathcal{A}(z), I}(n, x, y)$, is defined as the output pair of the honest party and the adversary $\mathcal{A}$ from the above ideal execution.

Execution in the Real Model In the real model, there is no trusted third party and the parties interact directly. The adversary $\mathcal{A}$ sends all messages in place of the corrupted party and may follow an arbitrary polynomial-time strategy. The honest parties follow the instructions of the specified protocol $\pi$.

Let $f$ be as above and let $\pi$ be a two-party protocol for computing $f$. Furthermore, let $\mathcal{A}$ be a PPT algorithm and let $I$ be the set of corrupted parties. Then, the real execution of $\pi$ on inputs $(x, y)$, auxiliary input $z$ to $\mathcal{A}$ and security parameter $n$, denoted $\operatorname{REAL}_{\pi, \mathcal{A}(z), I}(n, x, y)$, is defined as the output vector of the honest parties and the adversary $\mathcal{A}$ from the real execution of $\pi$.

Security as Emulation of a Real Execution in the Ideal Model Having defined the ideal and real models, we can now define security of protocols. Loosely speaking, the definition asserts that a secure party protocol (in the real model) emulates the ideal model (in which a trusted party exists). This is formulated by saying that adversaries in the ideal model are able to simulate executions of the real-model protocol.

Definition A.6. Let $f$ and $\pi$ be as above. Protocol $\pi$ is said to securely compute $f$ with abort in the presence of malicious adversaries if for every PPT adversary $\mathcal{A}$ for the real model, there exists a PPT adversary $\mathcal{S}$ for the ideal model, such that for every $I \subset[2]$, 


$$
\left\{\operatorname{IDEAL}_{f, \mathcal{S}(z), I}(n, x, y)\right\}_{n \in \mathbb{N}, x, y, z \in\{0,1\}^{*}} \stackrel{1 / \mathrm{p}}{\approx}\left\{\mathbf{R E A L}_{\pi, \mathcal{A}(z), I}(n, x, y)\right\}_{n \in \mathbb{N}, x, y, z \in\{0,1\}^{*}}
$$

where $n$ is the security parameter.

The $\mathcal{F}$-hybrid model. In order to construct some of our protocols, we will use secure two-party protocols as subprotocols. The standard way of doing this is to work in a "hybrid model" where parties both interact with each other (as in the real model) and use trusted help (as in the ideal model). Specifically, when constructing a protocol $\pi$ that uses a subprotocol for securely computing some functionality $\mathcal{F}$, we consider the case that the parties run $\pi$ and use "ideal calls" to a trusted party for computing $\mathcal{F}$. Upon receiving the inputs from the parties, the trusted party computes $\mathcal{F}$ and sends all parties their output. Then, after receiving these outputs back from the trusted party the protocol $\pi$ continues. Let $\mathcal{F}$ be a functionality and let $\pi$ be a two-party protocol that uses ideal calls to a trusted party computing $\mathcal{F}$. Furthermore, let $\mathcal{A}$ be a non-uniform probabilistic polynomial-time algorithm. Then, the $\mathcal{F}$-hybrid execution of $\pi$ on inputs $(x, y)$, auxiliary input $z$ to $\mathcal{A}$ and security parameter $n$, denoted $\operatorname{hyb}_{\pi} \mathcal{F}_{\mathcal{A}(z)}(n, x, y)$, is defined as the output vector of the honest parties and the adversary $\mathcal{A}$ from the hybrid execution of $\pi$ with a trusted party computing $\mathcal{F}$. By the composition theorem of [14] any protocol that securely implements $\mathcal{F}$ can replace the ideal calls to $\mathcal{F}$.

\section{B Proving Corollary 6.4}

In this section, we argue that our OT protocol from Sect. 6 and ensuing 2PC due to [43], satisfy the notion of input-indistinguishable computation (IIC) due to [51].

We first recall our protocol below:

1. Sen $\rightarrow \operatorname{Rec}$ Sen samples $\left(i, \mathrm{tk}^{0}, \mathrm{tk}^{1}\right) \leftarrow \operatorname{Gen}\left(1^{n}\right)$ and sends $i$ to the receiver Rec.

2. $\operatorname{Rec} \rightarrow$ Sen Rec samples $x \leftarrow D_{i}$ and sends $y=f_{i}^{b}(x)$.

3. Sen $\rightarrow$ Rec Upon receiving $y$, Sen computes $x_{\beta}=\left(f_{i}^{\beta}\right)^{-1}(y)$ for all $\beta \in\{0,1\}$, and sends $\left(\left\langle x_{0}, r_{0}\right\rangle \oplus s_{0}, r_{0}\right)$ and $\left(\left\langle x_{1}, r_{1}\right\rangle \oplus s_{1}, r_{1}\right)$ for random $r_{0}, r_{1} \cdot{ }^{14}$

To prove that our protocol satisfies IIC, we need to show there exist implicit input functions $I N_{1}$ and $I N_{2}$ that respectively satisfy implicit computation and input-indistinguishability for the sender and receiver. First, since the sender does not receive any output in the OT protocol, it follows immediately that implicit computation w.r.t the sender holds against a malicious receiver. On the other hand, input indistinguishability against a malicious sender follows since the receiver's message information theoretically hides its input bit. Next, we argue implicit computation w.r.t the receiver against a malicious sender. This follows from the fact that the functions $f_{i}^{b}$ are permutations and the third message perfectly binds the sender's inputs. Hence, it is possible to define $\mathrm{IN}_{1}$ for which the receiver learns the value corresponding to this input.

In order to argue input-indistinguishability against a malicious receiver, we need to show that there exists an implicit input function $\mathrm{IN}_{2}$ for which this property holds. However, this follows from the fact that if there does not exist any implicit input function for

\footnotetext{
${ }^{14}$ We can consider some canonical representation of elements in $D_{i}$ in $\{0,1\}^{*}$.
} 
which the property holds, then there must exist a malicious receiver $R^{*}$, polynomial $p(\cdot)$, infinitely many lengths $n$, and values $s_{0}^{n}, s_{1}^{n}, \widehat{s}_{0}^{n}, \widehat{s}_{1}^{n}, \widetilde{s}_{n}^{0}, \widetilde{s}_{n}^{1}$ such that $s_{1}^{n} \neq \widetilde{s}_{1}^{n}, \widehat{s}_{0}^{n} \neq \widetilde{s}_{0}^{n}$ and $R^{*}$ can distinguish with probability at least $\frac{1}{p(n)}$ in both the following cases:

1. Sender's inputs are $\left(s_{0}^{n}, s_{1}^{n}\right)$ and $\left(s_{0}^{n}, \widetilde{s}_{1}^{n}\right)$, and

2. Sender's inputs are $\left(\widehat{s}_{0}^{n}, \widehat{s}_{1}^{n}\right)$ and $\left(\widetilde{s}_{0}^{n}, \widehat{s}_{1}^{n}\right)$

In other words, the receiver can distinguish both the sender values (from random) for infinitely many lengths. This contradicts the fact that our protocol is private against a malicious receiver as proved in Theorem 6.2 (which in turn was proved by showing that $R^{*}$ can break the claw-freeness of the family of the functions).

\section{References}

[1] P. Ananth, A. R. Choudhuri, A. Jain, A new approach to round-optimal secure multiparty computation, in CRYPTO (2017), pp. 468-499

[2] Y. Aumann, Y. Lindell. Security against covert adversaries: Efficient protocols for realistic adversaries. J. Cryptology, 23(2). 281-343 (2010)

[3] N. Asokan, V. Shoup, M. Waidner, Optimistic fair exchange of digital signatures. IEEE J. Sel. Areas Commun. 18(4), 593-610 (2000)

[4] R. Bendlin, I. Damgård, C. Orlandi, S. Zakarias, Semi-homomorphic encryption and multiparty computation, in EUROCRYPT (2011), pp. 169-188

[5] D. Beaver, Foundations of secure interactive computing, in CRYPTO (1991), pp. 377-391

[6] S. Badrinarayanan, V. Goyal, A. Jain, Y. T. Kalai, D. Khurana, A. Sahai. Promise zero knowledge and its applications to round optimal mpc. IACR Cryptol. ePrint Arch. 2017, 1088 (2017)

[7] S. Badrinarayanan, V. Goyal, A. Jain, D. Khurana, A. Sahai, Round optimal concurrent MPC via strong simulation, in TCC (2017), pp. 743-775

[8] S. Badrinarayanan, V. Goyal, A. Jain, Y. T. Kalai, D. Khurana, A. Sahai, Promise zero knowledge and its applications to round optimal MPC, in CRYPTO (2018), pp. 459-487

[9] Z. Brakerski, S. Halevi, A. Polychroniadou, Four round secure computation without setup, in TCC (2017), pp. 645-677

[10] I. Bentov, R. Kumaresan, How to use bitcoin to design fair protocols, in CRYPTO (2014), pp. 421-439

[11] F. Benhamouda, H. Lin, k-round multiparty computation from k-round oblivious transfer via garbled interactive circuits, in EUROCRYPT (2018), pp. 500-532

[12] M. Blum, How to prove a theorem so no one else can claim it, in Proceedings of the International Congress of Mathematicians, USA, pp. 1444-1451

[13] B. Barak, A. Sahai, How to play almost any mental game over the net—concurrent composition via super-polynomial simulation. IACR Cryptol. ePrint Arch., 106 (2005)

[14] R. Canetti, Security and composition of multiparty cryptographic protocols. J. Cryptol. 13(1), 143-202 (2000)

[15] R. Cramer, I. Damgård, B. Schoenmakers, Proofs of partial knowledge and simplified design of witness hiding protocols, in CRYPTO (1994), pp. 174-187

[16] D. Chaum, J.-H. Evertse, J. van de Graaf, An improved protocol for demonstrating possession of discrete logarithms and some generalizations, in EUROCRYPT (1987), pp. 127-141

[17] R. Cleve, Limits on the security of coin flips when half the processors are faulty (extended abstract), in STOC (1986), pp. 364-369

[18] R. Canetti, H. Lin, R. Pass, Adaptive hardness and composable security in the plain model from standard assumptions, in FOCS (2010), pp. 541-550

[19] K.-M. Chung, E. Lui, R. Pass, From weak to strong zero-knowledge and applications, in TCC (2015), pp. 66-92

[20] M. Ciampi, R. Ostrovsky, L. Siniscalchi, I. Visconti, Round-optimal secure two-party computation from trapdoor permutations, in TCC (2017), pp. 678-710 
[21] J. Doerner, Y. Kondi, E. Lee, A. Shelat. Secure two-party threshold ECDSA from ECDSA assumptions, in IEEE Symposium on Security and Privacy, SP (2018), pp. 980-997

[22] I. Damgård, V. Pastro, N. P. Smart, S. Zakarias, Multiparty computation from somewhat homomorphic encryption, in CRYPTO (2012), pp. 643-662

[23] S. Even, O. Goldreich, A. Lempel. A randomized protocol for signing contracts. Commun. ACM 28(6), 637-647 (1985)

[24] M. Fischlin, Trapdoor commitment schemes and their applications. Ph.D. Thesis (2001)

[25] T. K. Frederiksen, Y. Lindell, V. Osheter, B. Pinkas, Fast distributed RSA key generation for semi-honest and malicious adversaries, in CRYPTO (2018), pp. 331-361

[26] U. Feige, A. Shamir, Witness indistinguishable and witness hiding protocols, in STOC (1990), pp. 416-426

[27] T. El Gamal, A public key cryptosystem and a signature scheme based on discrete logarithms. IEEE Trans. Inf. Theory, 31(4), 469-472 (1985)

[28] O. Goldreich, H. Krawczyk, On the composition of zero-knowledge proof systems. SIAM J. Comput., 25(1), 169-192 (1996)

[29] S. Dov Gordon, J. Katz, Partial fairness in secure two-party computation, in EUROCRYPT (2010), pp. $157-176$

[30] J. A. Garay, J. Katz, B. Tackmann, V. Zikas, How fair is your protocol?: A utility-based approach to protocol optimality, in PODC (2015), pp. 281-290

[31] O. Goldreich, L. A. Levin, A hard-core predicate for all one-way functions, in STOC (1989), pp. 25-32

[32] S. Garg, P. Mukherjee, O. Pandey, A. Polychroniadou, The exact round complexity of secure computation. In M. Fischlin, J.S. Coron, editors, Advances in Cryptology - EUROCRYPT, 35th Annual International Conference on the Theory and Applications of Cryptographic Techniques, Vienna, Austria, May 8-12, 2016, Proceedings, Part II, pages 448-476. Springer, Berlin, Heidelberg (2016)

[33] S. Goldwasser, S. Micali, R. L. Rivest, A "paradoxical” solution to the signature problem (extended abstract), in FOCS (1984), pp. 441-448

[34] O. Goldreich, S. Micali, A. Wigderson, How to play any mental game or a completeness theorem for protocols with honest majority, in STOC (1987), pp. 218-229

[35] O. Goldreich, Foundations of cryptography: Vol. 2, Basic Applications (Cambridge University Press, New York, 2004)

[36] S. Garg, A. Srinivasan, Two-round multiparty secure computation from minimal assumptions, in $E U$ ROCRYPT (2018), pp. 468-499

[37] I. Haitner, Semi-honest to malicious oblivious transfer - the black-box way, in TCC (2008), pp. 412-426

[38] S. Halevi, C. Hazay, A. Polychroniadou, M. Venkitasubramaniam. Round-optimal secure multi-party computation, in CRYPTO (2018), pp. 488-520

[39] I. Haitner, Y. Ishai, E. Kushilevitz, Y. Lindell, E. Petrank, Black-box constructions of protocols for secure computation. SIAM J. Comput. 40(2), 225-266 (2011)

[40] S. Halevi, Y. T. Kalai, Smooth projective hashing and two-message oblivious transfer. J. Cryptol. 25(1), 158-193 (2012)

[41] C. Hazay, G. L. Mikkelsen, T. Rabin, T. Toft, Efficient RSA key generation and threshold Paillier in the two-party setting, in CT-RSA (2012), pp. 313-331

[42] C. Hazay, P. Scholl, E. Soria-Vazquez, Low cost constant round MPC combining BMR and oblivious transfer, in ASIACRYPT (2017), pp. 598-628

[43] Y. Ishai, E. Kushilevitz, R. Ostrovsky, M. Prabhakaran, A. Sahai, Efficient non-interactive secure computation, in EUROCRYPT (2011), pp. 406-425

[44] Y. Ishai, E. Kushilevitz, R. Ostrovsky, A. Sahai, Zero-knowledge proofs from secure multiparty computation. SIAM J. Comput. 39(3), 1121-1152 (2009)

[45] J. Katz, R. Ostrovsky, Round-optimal secure two-party computation, in CRYPTO (2004), pp. 335-354

[46] M. Keller, E. Orsini, P. Scholl, MASCOT: faster malicious arithmetic secure computation with oblivious transfer, in CCS (2016), pp. 830-842

[47] Y. Lindell, Parallel coin-tossing and constant-round secure two-party computation, in CRYPTO (2001), pp. 171-189

[48] Y. Lindell, A. Nof, Fast secure multiparty ECDSA with practical distributed key generation and applications to cryptocurrency custody, in CCS (2018), pp. 1837-1854

[49] S. Micali, Simple and fast optimistic protocols for fair electronic exchange, in PODC (2003), pp. 12-19 
[50] T. Moran, M. Naor, G. Segev, An optimally fair coin toss, in TCC (2009), pp. 1-18

[51] S. Micali, R. Pass, A. Rosen, Input-indistinguishable computation, in FOCS (2006), pp. 367-378

[52] S. Micali, P. Rogaway, Secure computation (abstract), in CRYPTO (1991), pp. 392-404

[53] J. B. Nielsen, P. S. Nordholt, C. Orlandi, S. S. Burra, A new approach to practical active-secure two-party computation, in CRYPTO (2012), pp. 681-700

[54] M. Naor, B. Pinkas, Efficient oblivious transfer protocols, in SODA (2001), pp. 448-457

[55] R. Ostrovsky, S. Richelson, A. Scafuro, Round-optimal black-box two-party computation, in CRYPTO (2015), pp. 339-358

[56] R. Pass, Simulation in quasi-polynomial time, and its application to protocol composition, in EUROCRYPT (2003), pp. 160-176

[57] T. P. Pedersen, Non-interactive and information-theoretic secure verifiable secret sharing, in CRYPTO (1991), pp. 129-140

[58] M. Prabhakaran, A. Sahai, New notions of security: achieving universal composability without trusted setup, in STOC (2004), pp. 242-251

[59] C. Peikert, V. Vaikuntanathan, B. Waters, A framework for efficient and composable oblivious transfer, in CRYPTO (2008), pp. 554-571

[60] R. Pass, H. Wee, Black-box constructions of two-party protocols from one-way functions, in TCC (2009), pp. $403-418$

[61] A. Shamir, How to share a secret. Commun. ACM 22(11), 612-613 (1979)

[62] A. C.-C. Yao, Theory and applications of trapdoor functions (extended abstract), in FOCS (1982), pp. 80-91

[63] A. C.-C. Yao, How to generate and exchange secrets (extended abstract), in FOCS (1986), pp. 162-167

Publisher's Note Springer Nature remains neutral with regard to jurisdictional claims in published maps and institutional affiliations. 